\title{
KOSZUL DUALITY PATTERNS IN REPRESENTATION THEORY
}

ALEXANDER BEILINSON, VICTOR GINZBURG, AND WOLFGANG SOERGEL

\section{CONTENTs}

1. Introduction 473

2. Koszul rings 479

3. Parabolic-singular duality and Koszul duality 496

4. Koszul rings arising from 'mixed' geometry 513

References $\quad 525$

\section{INTRODUCTION}

1.1. Parabolic-singular duality. Let $\mathfrak{g} \supset \mathfrak{b} \supset \mathfrak{h}$ be a complex semisimple Lie algebra, a Borel subalgebra and a Cartan subalgebra contained in it, respectively. Let $\mathrm{U}=\mathrm{U}(\mathfrak{g})$ be the universal enveloping algebra of $\mathfrak{g}$. Let $\mathcal{O}$ be the category of all finitely generated U-modules which are locally finite over $\mathfrak{b}$ and semisimple over $\mathfrak{h}$, see for example [BG80]. For $\lambda \in \mathfrak{h}^{*}$ consider in $\mathcal{O}$ the Verma module $M(\lambda)=\mathrm{U} \otimes_{\mathrm{U}(\mathfrak{b})} \mathbb{C}_{\lambda}$, its simple quotient $L(\lambda)$ and the (indecomposable) projective cover $P(\lambda)$ of $L(\lambda)$ in $\mathcal{O}$.

Let $Z \subset \mathrm{U}$ be the center. Let $L \in \mathcal{O}$ be the direct sum of all simple highest weight modules $L(\lambda)$ which have trivial infinitesimal character, i.e. which have the same annihilator in $Z$ as the trivial representation $\mathbb{C}=L(0)$. There are but finitely many of those, parametrized by the Weyl group. Let $P \in \mathcal{O}$ be the direct sum of their respective projective covers. The first statement of the following theorem is the main result of [Soe90].

Theorem 1.1.1. There exists an isomorphism of finite dimensional $\mathbb{C}$-algebras

$$
\operatorname{End}_{\mathcal{O}} P \cong \operatorname{Ext}_{\mathcal{O}}^{\bullet}(L, L)
$$

where $\mathrm{Ext}^{\bullet}$ stands for the direct sum of all $\mathrm{Ext}^{i}$, made into a ring via the cup product. Furthermore, $\operatorname{Ext}_{\mathcal{O}}^{\bullet}(L, L)$ is a Koszul ring.

We have to explain what a Koszul ring is.

Received by the editors November 13, 1991 and, in revised form, February 16, 1995.

1991 Mathematics Subject Classification. Primary 17B10; Secondary 16A03.

The first author was partially supported by an NSF grant.

The second author thanks Harvard University and MIT, where part of this work was written.

The third author thanks the MPI and DFG for financial support. 
Definition 1.1.2. A Koszul ring is a positively graded ring $A=\bigoplus_{j \geq 0} A_{j}$ such that (1) $A_{0}$ is semisimple and (2) $A_{0}$ considered as a graded left $A$-module admits a graded projective resolution

$$
\cdots \rightarrow P^{2} \rightarrow P^{1} \rightarrow P^{0} \rightarrow A_{0}
$$

such that $P^{i}$ is generated by its degree $i$ component, i.e., $P^{i}=A P_{i}^{i}$.

Morally a Koszul ring is a graded ring that is "as close to semisimple as a $\mathbb{Z}$-graded ring possibly can be". This statement will be made more precise by Proposition 2.1.3.

We explain next the meaning of the $\operatorname{ring} \mathrm{E}=\operatorname{End}_{\mathcal{O}} P$. If $\mathcal{A}$ is an abelian category whose objects have finite length and $P \in \mathcal{A}$ a projective generator of $\mathcal{A}$ (i.e. a projective object surjecting onto each simple object in $\mathcal{A}$ ), then there is a canonical standard (see, e.g., [Bas68], the exercise following Theorem 1.3 from Chapter II) equivalence of categories

$$
\operatorname{Hom}_{\mathcal{A}}(P, \quad): \mathcal{A} \rightarrow \text { Mof-E, } \quad \mathrm{E}=\operatorname{End}_{\mathcal{A}} P,
$$

where Mof- stands for the category of finitely generated right modules. Now let $Z^{+}=\operatorname{Ann}_{Z} \mathbb{C} \subset Z$ be the augmentation ideal of the center. Apply the considerations above to the category $\mathcal{O}_{0}=\left\{M \in \mathcal{O} \mid\left(Z^{+}\right)^{n} M=0\right.$ for $\left.n \gg 0\right\}$. Thus we get an equivalence of categories

$$
\mathcal{O}_{0} \cong \operatorname{Mof}-\left(\operatorname{End}_{\mathcal{O}} P\right) \text {. }
$$

So the theorem says in particular that the category $\mathcal{O}_{0}$ is governed by a Koszul ring, i.e. that it is in some sense "graded semisimple". We hope to convince our readers that this is a good way to formalize the beauty of this part of representation theory, and we believe that similar results should hold for all kinds of representation theories associated to root systems.

As a first step we explain how to generalize the above to other infinitesimal characters. Let $\rho \in \mathfrak{h}^{*}$ be the half sum of positive roots, i.e. of the roots in $\mathfrak{b}$. Let $(\mathcal{W}, \mathcal{S})$ be the Coxeter system of $\mathfrak{g} \supset \mathfrak{b}$ and let $w_{\circ}$ be the longest element of the Weyl group $\mathcal{W}$. For $w \in \mathcal{W}, \lambda \in \mathfrak{h}^{*}$ we put $w \cdot \lambda=w(\lambda+\rho)-\rho$. For any subset $\mathcal{S}_{\iota} \subset \mathcal{S}$-here $\iota$ is a variable index-let $\mathcal{W}_{\iota} \subset \mathcal{W}$ be the subgroup generated by $\mathcal{S}_{\iota}$, $w_{\iota} \in \mathcal{W}_{\iota}$ its longest element and $\mathcal{W}^{\iota} \subset \mathcal{W}$ the set of longest representatives of the cosets $\mathcal{W} / \mathcal{W}_{\iota}$.

For $\lambda \in \mathfrak{h}^{*}$ integral dominant but perhaps singular (in other words, $\lambda$ is integral and $\lambda+\rho$ lies in the closure of the dominant Weyl chamber) define the category $\mathcal{O}_{\lambda} \subset \mathcal{O}$ to consist of all objects of $\mathcal{O}$ whose (generalized) infinitesimal character coincides with the one of $L(\lambda)$. Set $\mathcal{S}_{\lambda}=\{s \in \mathcal{S} \mid s \cdot \lambda=\lambda\}$. Then $\{L(x \cdot \lambda)\}_{x \in \mathcal{W}^{\lambda}}$ represents the isomorphism classes of simple objects in $\mathcal{O}_{\lambda}$. The $P(x \cdot \lambda)$ are the projective covers of $L(x \cdot \lambda)$ in $\mathcal{O}_{\lambda}$ as well.

For $\mathfrak{q} \subset \mathfrak{g}$ a parabolic subalgebra containing $\mathfrak{b}$ define the subcategory $\mathcal{O}^{\mathfrak{q}} \subset \mathcal{O}_{0}$ to consist of all $\mathfrak{q}$-locally finite objects. Let $\mathcal{S}_{\mathfrak{q}} \subset \mathcal{S}$ be the simple reflections corresponding to $\mathfrak{q}$. For all $x \in \mathcal{W}^{\mathfrak{q}}$ we set $L_{x}^{\mathfrak{q}}=L\left(x^{-1} w_{\circ} \cdot 0\right) \in \mathcal{O}^{\mathfrak{q}}$. The $L_{x}^{\mathfrak{q}}$ represent the isomorphism classes of simple objects in $\mathcal{O}^{\mathfrak{q}}$. Any $L_{x}^{\mathfrak{q}}$ has a projective cover $P_{x}^{\mathfrak{q}}$ in $\mathcal{O}^{\mathfrak{q}}$.

We will prove in section 3 
Theorem 1.1.3. Let $\lambda, \mathfrak{q}$ be as above and suppose $\mathcal{S}_{\lambda}=\mathcal{S}_{\mathfrak{q}}$. Then there are isomorphisms of finite dimensional $\mathbb{C}$-algebras

$$
\begin{array}{ll}
\operatorname{End}_{\mathcal{O}_{\lambda}}(\bigoplus P(x \cdot \lambda)) & \cong \operatorname{Ext}_{\mathcal{O}_{\mathfrak{q}}}^{\bullet}\left(\bigoplus L_{x}^{\mathfrak{q}}, \oplus L_{x}^{\mathfrak{q}}\right), \\
\operatorname{End}_{\mathcal{O}_{\mathfrak{q}}}\left(\bigoplus P_{x}^{\mathfrak{q}}\right) & \cong \operatorname{Ext}_{\mathcal{O}_{\lambda}}(\bigoplus L(x \cdot \lambda), \bigoplus L(x \cdot \lambda)),
\end{array}
$$

where the sums go over $x \in \mathcal{W}^{\lambda}=\mathcal{W}^{\mathfrak{q}}$. Both algebras on the right are Koszul rings and, moreover, these rings are Koszul dual to one another.

Remarks. (1) We have to explain when two Koszul rings are dual to one another. For every positively graded $\operatorname{ring} A$ we may consider $A_{0}$ as a left $A$-module $A_{0}=A / A_{>0}$ and form the positively graded ring $E(A)=\operatorname{Ext}_{A}^{\bullet}\left(A_{0}, A_{0}\right)$. For a Koszul ring $A$ satisfying a suitable finiteness condition we prove as Theorem 2.10.2 that $E(A)$ is Koszul as well and $E(E(A))=A$ canonically. Motivated by this equality, we call $E(A)$ the dual of $A$.

(2) Remark that $\mathcal{O}^{\mathfrak{b}}=\mathcal{O}_{0}$. So the theorem tells us in particular that the Koszul ring from Theorem 1.1.1 is selfdual.

(3) All sums go over $x \in \mathcal{W}^{\mathfrak{q}}=\mathcal{W}^{\lambda}$. Let $1_{x}$ be the obvious pairwise orthogonal idempotents in our four algebras. The isomorphisms of the theorem can (and will) be chosen so that the $1_{x}$ correspond to each other under the isomorphisms above.

(4) From what we explained before it is clear that the endomorphism algebras of projectives appearing in the theorem govern the categories $\mathcal{O}_{\lambda}$ and $\mathcal{O}^{\mathfrak{q}}$ respectively.

(5) The projective covers appearing in the theorem are unique only up to nonunique isomorphism, hence we cannot expect the isomorphisms predicted by the theorem to be canonical. We can however construct without choices a collection of possible $P(x \cdot \lambda)$ along with a canonical isomorphism as in the theorem for this special collection.

(6) Morally we should rather consider categories $\mathcal{O}_{\lambda}$ and $\mathcal{O}^{\mathfrak{q}}$ for Langlands-dual Lie algebras. In fact one might then view $\mathcal{O}_{\lambda}$ as a category of Harish-Chandra modules for a complex group and $\mathcal{O}^{\mathfrak{q}}$ as a category of perverse sheaves on the corresponding space of Langlands parameters, more precisely its modified version defined by Adams-Barbasch-Vogan [ABV92]. Our theorem can then be understood as a refinement of Langlands' philosophy in the case of complex groups. More details on this point of view can be found in [Soe92].

This article is the result of our efforts to join the two partially wrong and quite incomplete preprints [Soe89b] and [BG86] into one readable article.

First we approach the above results in as elementary a way as possible. The only facts relying on "mixed" geometry we really use are the computation of the intersection cohomology of Schubert varieties and Lemma 3.5.4. In the last section we adopt the more powerful technique of mixed geometry.

1.2. Koszul duality. The results described here and proved in section 2 are essentially not new but rather generalizations of results of Priddy [Pri70], Löfwall [Löf86], Backelin [Bac82], Backelin-Fröberg [BF85], Bernstein-Gelfand-Gelfand [BGG78] and specializations of results from [BGS88]. We start by repeating our definition of a Koszul ring.

Definition 1.2.1. A Koszul ring is a positively graded $\operatorname{ring} A=\bigoplus_{j \geq 0} A_{j}$ such that (1) $A_{0}$ is semisimple and (2) $A_{0}$ considered as a graded left $A$-module admits 
a graded projective resolution

$$
\cdots \rightarrow P^{2} \rightarrow P^{1} \rightarrow P^{0} \rightarrow A_{0}
$$

such that $P^{i}$ is generated by its component of degree $i, P^{i}=A P_{i}^{i}$.

Remarks. (1) A complex of graded $A$-modules

$$
\cdots \rightarrow P^{2} \rightarrow P^{1} \rightarrow P^{0} \rightarrow A_{0}
$$

as in the definition is unique up to unique isomorphism and has a natural right $A_{0}$-action. This follows from the obvious fact that if $Q^{\bullet}$ is another resolution of $A_{0}$ as in the definition, then any chain map $P^{\bullet} \rightarrow Q^{\bullet}$ which respects the grading on the $P^{i}, Q^{i}$ and is homotopic to zero must itself be zero.

(2) Let $V$ be a finite dimensional vector space over a field $k$. Then the symmetric algebra $S V$ is Koszul, as can be seen from the Koszul complex

$$
\cdots \rightarrow S V \otimes \bigwedge^{2} V \rightarrow S V \otimes V \rightarrow S V \rightarrow k .
$$

(3) We will prove in section 2.2 that $A$ is Koszul if and only if $A^{\text {opp }}$ is.

Definition 1.2.2. A quadratic ring is a positively graded ring $A=\bigoplus_{j \geq 0} A_{j}$ such that $A_{0}$ is semisimple and $A$ is generated over $A_{0}$ by $A_{1}$ with relations of degree two.

This may be spelled out as follows: Let

$$
T_{A_{0}} A_{1}=A_{0} \oplus A_{1} \oplus\left(A_{1} \otimes_{A_{0}} A_{1}\right) \oplus \ldots=\bigoplus_{i \geq 0} A_{1}^{\otimes i}
$$

be the free tensor algebra of the $A_{0}$-bimodule $A_{1}$. Then the canonical map can : $T_{A_{0}} A_{1} \rightarrow A$ is required to be a surjection and $\operatorname{ker}(\mathrm{can})$ is required to be generated, as a two-sided ideal, by its component $R=\operatorname{ker}(\operatorname{can}) \cap\left(A_{1} \otimes_{A_{0}} A_{1}\right)$ of degree two, $\operatorname{ker}(\operatorname{can})=(R)$, so that $T_{A_{0}} A_{1} /(R)=A$. In section 2.3 we show:

Proposition 1.2.3. Any Koszul ring is quadratic.

Let us remark that for any semisimple ring $k$ and any $k$-bimodule $V$ the quadratic ring with no relations, $T_{k} V$, is Koszul. To have duality we need some finiteness conditions.

Definition 1.2.4. A graded $\operatorname{ring} A=\oplus_{i} A_{i}$ is called "left finite" (resp. "right finite") if and only if all $A_{i}$ are finitely generated as left (resp. right) $A_{0}$-modules.

For any positively graded ring $A=\bigoplus_{j \geq 0} A_{j}$ consider now the left $A$-module $A_{0}$ and form the graded ring $E(A)=\operatorname{Ext}_{A}^{\bullet}\left(A_{0}, A_{0}\right)$ of self-extensions of $A_{0}$. We will prove as Theorem 2.10 .2

Theorem 1.2.5. Suppose $A$ is a left finite Koszul ring. Then $E(A)$ is a left finite Koszul ring as well, and $E(E(A))=A$ canonically.

In the situation of the theorem we say that the Koszul rings $A$ and $E(A)$ are "dual to each other". For example, the Koszul dual of the symmetric algebra $S V$ above is the exterior algebra $\bigwedge V^{*}$ on the dual space of $V$, and for a bimodule $V$ over a semisimple ring $k$ that is finitely generated for the left $k$-action the Koszul dual of $T_{k} V$ is $\left(k \oplus \operatorname{Hom}_{k}(V, k)\right)^{\text {opp }}$. The theorem is proved by identifying $E(A)$ with the opposed ring of the more elementary quadratic dual ring $A^{!}$of $A$, which we will however not define in this introduction. 
Next we explain Koszul duality at the level of derived categories. To do so we need more notations. For a graded ring $A=\bigoplus_{j} A_{j}$ let $A$-Mod denote the category of all left $A$-modules $M$ and $A$-mod the category of all graded left $A$ modules $M=\bigoplus_{j} M_{j}$. Let $\operatorname{Hom}_{A}($ resp. hom $A$ ) denote homomorphisms in $A$-Mod (resp. $A$-mod). So $\operatorname{hom}_{A}(M, N)=\left\{f \in \operatorname{Hom}_{A}(M, N) \mid f\left(M_{j}\right) \subset N_{j}\right\}$. We define the grading shifts $\langle n\rangle$ by $(M\langle n\rangle)_{j}=M_{j-n}$. The respective subcategories of finitely generated (graded) modules will be denoted $A$-Mof and $A$-mof. These are abelian categories provided $A$ is left noetherian.

For any abelian category $\mathcal{A}$ let $D^{b}(\mathcal{A})$ be the corresponding bounded derived category. It comes equipped with the structure of a triangulated category, in particular with a shift functor $[1]: D^{b}(\mathcal{A}) \rightarrow D^{b}(\mathcal{A})$. It also comes with a canonical degree zero embedding $\mathcal{A} \rightarrow D^{b}(\mathcal{A})$.

Now let $A$ be a Koszul ring that satisfies the following two finiteness conditions: (1) $A$ is a finitely generated $A_{0}$-module from the left as well as from the right, in particular $A_{i}=0$ for all $i \gg 0 ;(2) E(A)$ is right noetherian. Put $A^{!}=E(A)^{\text {opp }}$. Then $A_{0}^{!}=A_{0}$ canonically, and we will denote this semisimple ring by $k$. We will also need the injective graded $A$-module $A^{*}=\operatorname{Hom}_{k}(A, k)$. Remark that it comes with a right $k$-action.

Under the above assumptions (1) and (2) we will construct in section 2.12 (in particular Theorem 2.12.6) an exact functor between triangulated categories

$$
K: D^{b}(A \text {-mof }) \rightarrow D^{b}\left(A^{!} \text {-mof }\right)
$$

(the "Koszul duality functor") and prove:

Theorem 1.2.6. The functor $K$ is an equivalence of triangulated categories. We have $K(M\langle n\rangle)=(K M)[-n]\langle-n\rangle$ canonically for $M \in D^{b}$ (A-mof). Furthermore, for all $p \in k$ we have $K\left(A_{0} p\right)=A^{!} p, K\left(A^{*} p\right)=A_{0}^{!} p$.

Remarks. (1) The last statement is to be understood via the embeddings $A$-mof $\rightarrow$ $D^{b}$ (A-mof), $A^{!}$-mof $\rightarrow D^{b}\left(A^{!}\right.$-mof $)$. Note also that any simple object of $A$-mof (resp. $A^{!}$-mof) has up to a grading shift the form $A_{0} p$ (resp. $A_{0}^{!} p$ ) for suitable $p \in k$. Furthermore, $A^{!} p$ is the projective cover of $A_{0}^{!} p$ and $A^{*} p$ the injective hull of $A_{0} p$ for any $p \in k$. So roughly speaking Koszul duality transforms simples to indecomposable projectives and indecomposable injectives to simples.

(2) For a Koszul ring $A$ one might interpret $E(A)$ as being $\operatorname{RHom}_{A}(k, k)$, and then the Koszul duality functor $K$ is just the functor $\operatorname{RHom}_{A}(k$,$) . We will$ however not pursue this perspective in our paper. More details on this point of view can be found in [Kel92].

(3) In the case of the symmetric algebra the equivalence

$$
D^{b}\left(\bigwedge V^{*} \text {-mof }\right) \cong D^{b}(S V \text {-mof })
$$

was established in [BGG78].

1.3. Parabolic-singular duality and Koszul duality. Let us start with an application. As explained in section 2.4, every module of finite length $M$ has a radical filtration

$$
M=\operatorname{rad}^{0} M \supset \operatorname{rad}^{1} M \supset \operatorname{rad}^{2} M \supset \ldots
$$

with semisimple subquotient $\overline{\operatorname{rad}} d^{i} M=\operatorname{rad}^{i} M / \operatorname{rad}^{i+1} M$ called the $i$-th layer of the radical filtration. For $x \in \mathcal{W}^{\mathfrak{q}}$ let $M_{x}^{\mathfrak{q}} \in \mathcal{O}^{\mathfrak{q}}$ be the corresponding parabolic Verma module, i.e. the maximal locally $\mathfrak{q}$-finite quotient of $M\left(x^{-1} w_{\circ} \cdot 0\right)$. 
Proposition 1.3.1. Let $\lambda, \mathfrak{q}$ be as in Theorem 1.1.3 and $x, y \in \mathcal{W}^{\mathfrak{q}}=\mathcal{W}^{\lambda}$. Then we have

$$
\begin{array}{ll}
\operatorname{dim}_{\mathbb{C}} \operatorname{Ext}_{\mathcal{O}}^{i}(M(x \cdot \lambda), L(y \cdot \lambda)) & =\left[\overline{\operatorname{rad}}^{i} M_{x}^{\mathfrak{q}}: L_{y}^{\mathfrak{q}}\right], \\
\operatorname{dim}_{\mathbb{C}} \operatorname{Ext}_{\mathcal{O}^{\mathfrak{q}}}^{i}\left(M_{x}^{\mathfrak{q}}, L_{y}^{\mathfrak{q}}\right) & =\left[\operatorname{rad}^{i} M(x \cdot \lambda): L(y \cdot \lambda)\right] .
\end{array}
$$

This can be proved without much trouble by expressing both sides in terms of coefficients of Kazhdan-Lusztig polynomials. Our approach is different, however. Not only it is independent of the combinatorics of the Kazhdan-Lusztig polynomials, but it provides a concrete meaning to the numerical formulas above.

Namely, fix $\lambda, \mathfrak{q}$ as in Theorem 1.1.3 and denote the graded algebras there by $A_{Q}$ and $A^{Q}$ respectively. Using the ordinary duality on the category $\mathcal{O}$, it is easy to see that both $A_{Q}$ and $A^{Q}$ are their own opposed algebras, and even to construct isomorphisms $A_{Q}=A_{Q}^{\mathrm{opp}}, A^{Q}=\left(A^{Q}\right)^{\mathrm{opp}}$. Now by Theorem 1.1.3 we know that $A^{Q}$ and $A_{Q}$ are Koszul dual to one another, $A^{Q} \cong E\left(A_{Q}\right)$. So the Koszul duality functor gives us an equivalence of triangulated categories

$$
D^{b}\left(A_{Q^{-} \text {mof }}\right) \cong D^{b}\left(A^{Q} \text {-mof }\right)
$$

But we know also from Theorem 1.1.3 and Remark 4 following it that

$$
\begin{aligned}
& \mathcal{O}^{q} \cong A^{Q} \text {-Mof, } \\
& \mathcal{O}_{\lambda} \cong A_{Q^{-M o f} .}
\end{aligned}
$$

In view of that we consider $A_{Q^{-}}$-mof and $A^{Q}$-mof as "graded versions" of $\mathcal{O}_{\lambda}$ and $\mathcal{O}^{\mathfrak{q}}$ respectively, and to emphasize this we denote them by

$$
\begin{aligned}
& \mathcal{O}^{Q}=A^{Q} \text {-mof }, \\
& \mathcal{O}_{Q}=A_{Q} \text {-mof. }
\end{aligned}
$$

With this change in notation our equivalence reads

$$
D^{b}\left(\mathcal{O}_{Q}\right) \cong D^{b}\left(\mathcal{O}^{Q}\right) \text {. }
$$

As any Koszul duality, this transforms indecomposable injectives to simples and simples to indecomposable projectives. We prove that it also transforms (graded versions of) dual Vermas in $\mathcal{O}_{Q}$ to (graded versions of) parabolic Vermas in $\mathcal{O}^{Q}$. In this framework the equalities of the proposition can then be understood as manifestations of Koszul duality. These ideas are developed section 3.11.

1.4. Koszul rings arising from mixed geometry. The purpose of the third chapter is to show how Koszul rings arise naturally in the framework of "mixed" geometry. The latter stands either for the theory of "mixed complexes" on an algebraic variety over a finite field, as introduced in [BBD82], or for the theory of "mixed Hodge modules" on a complex algebraic variety, as developed by Saito [Sai90]. To make this part of the introduction accessible to a non-expert, we shall formulate our results without the language of mixed geometry first, and then explain how the mixed geometry enters the game.

Thus, we start with a complex algebraic variety $X$ equipped with an algebraic Whitney stratification $X=\bigcup X_{w}, w \in \mathcal{W}$, where $\mathcal{W}$ is a finite set indexing the strata. In the rest of the introduction we assume that each stratum $X_{w}$ is a locallyclosed algebraic subvariety of $X$ isomorphic to an affine linear space. Let $D^{b}(X)$ denote the bounded derived category of algebraically constructible complexes of $\mathbb{C}$ sheaves on $X, D^{b}(X, \mathcal{W}) \subset D^{b}(X)$ the full triangulated subcategory whose objects are all complexes $\mathcal{F}$ such that the cohomology sheaves $\mathcal{H}^{\bullet} \mathcal{F}$ are constant along 
the strata, and $\mathcal{P}(X, \mathcal{W}) \subset D^{b}(X, \mathcal{W})$ the subcategory of all perverse sheaves in $D^{b}(X, \mathcal{W})$. Thus, $\mathcal{P}(X, \mathcal{W})$ is an abelian subcategory of $D^{b}(X, \mathcal{W})$. The strata being affine, it follows that for each $w \in \mathcal{W}$, the imbedding $j_{w}: X_{w} \hookrightarrow X$ is affine. Hence, $\Delta_{w}=\left(j_{w}\right)_{!}\left(\mathbb{C}_{X_{w}}\right)\left[\operatorname{dim} X_{w}\right], \nabla_{w}=\left(j_{w}\right)_{*}\left(\mathbb{C}_{X_{w}}\right)\left[\operatorname{dim} X_{w}\right]$ are welldefined objects of $\mathcal{P}(X, \mathcal{W})$. We first prove the following result, which was obtained earlier, in [MV87], by different methods.

Proposition 1.4.1. (1) Each object of $\mathcal{P}(X, \mathcal{W})$ has a finite projective resolution. In particular, the category $\mathcal{P}(X, \mathcal{W})$ has enough projectives.

(2) The category $\mathcal{P}(X, \mathcal{W})$ is equivalent to the category of finite-dimensional modules over a finite-dimensional $\mathbb{C}$-algebra.

(3) Any projective in $\mathcal{P}(X, \mathcal{W})$ has a finite filtration with all successive subquotients being isomorphic to $\Delta_{w}$ for various $w \in \mathcal{W}$.

Let $\bar{X}_{w}$ denote the closure of a stratum $X_{w}, w \in \mathcal{W}$, and $\mathcal{L}_{w}=\operatorname{IC}\left(\mathbb{C}_{X_{w}}\right)$ the intersection cohomology complex. The intersection complexes $\mathcal{L}_{w}, w \in \mathcal{W}$ (extended by zero to the whole of $X$ ), are precisely the simple objects of the category $\mathcal{P}(X, \mathcal{W})$. For $y \in \mathcal{W}$, the restriction $j_{y}^{*}\left(\mathcal{L}_{w}\right)$ is a complex with constant cohomology sheaves $\mathcal{H}^{i}\left(j_{y}^{*} \mathcal{L}_{w}\left[-\operatorname{dim} X_{y}\right]\right)=n_{y, w}^{i} \cdot \mathbb{C}_{X_{y}}$ where $n_{y, w}^{i} \in \mathbb{N}$ are certain multiplicities which vanish for positive $i$. Hence, we may introduce a $\mathbb{Z}[t]$-valued $\mathcal{W} \times \mathcal{W}$-matrix $I C$ with entries $I C_{y, w}=\sum_{i \leq 0} n_{y, w}^{i} \cdot t^{-i}$.

From now on we make the following additional assumption on the stratification $X=\bigcup X_{w}$ :

$(*)$ : For each $w \in \mathcal{W}$, there exists a resolution of singularities $\hat{X}_{w} \rightarrow \bar{X}_{w}$ (of the closure of $X_{w}$ ) such that the rational homology of $\hat{X}_{w}$ is spanned by algebraic cycles.

The main results of the chapter are summarized in the following theorem.

Theorem 1.4.2. If the condition (*) holds, then:

(1) The category $\mathcal{P}(X, \mathcal{W})$ is equivalent to A-Mof, the category of finite dimensional modules over a finite dimensional graded Koszul $\mathbb{C}$-algebra $A=$ $\bigoplus_{i \geq 0} A_{i}$ where $A_{0}=\mathbb{C} \oplus \ldots \oplus \mathbb{C}(\# \mathcal{W}$ times $)$.

(2) There is a graded algebra isomorphism

$$
A^{!}=\operatorname{Ext}_{D^{b}(X)}^{\bullet}(\mathcal{L}, \mathcal{L}), \quad \mathcal{L}:=\bigoplus_{w \in \mathcal{W}} \mathcal{L}_{w} .
$$

In particular, the Ext-algebra above is Koszul.

(3) There is a matrix identity: $P\left(A^{!}, t\right)=I C^{T} \cdot I C$.

Observe that we claim that $\mathcal{P}(X, \mathcal{W})=A$-Mof is the category of non-graded $A$ modules. Now we may explain how the mixed geometry enters the game. Namely, the mixed version of the category $\mathcal{P}(X, \mathcal{W})$ is equivalent to the category $A$-mof of graded $A$-modules. This gives a geometric explanation of the graded setting.

\section{KosZUL RINGS}

2.1. An alternative definition of Koszul rings. Recall that $A$-mod denotes the category of graded modules, as opposed to $A$-Mod for the category of ordinary modules over $A$. Both of these are abelian categories, and we will denote morphisms and extensions in the graded category by $\operatorname{hom}_{A}, \operatorname{ext}_{A}$ as opposed to $\mathrm{Hom}_{A}, \operatorname{Ext}_{A}$. 
Definition 2.1.1. A graded module $M$ over a graded ring is called "pure of weight $m$ " if and only if it is concentrated in degree $-m$, i.e. $M=M_{-m}$.

Suppose $A$ is a positively graded ring such that $A_{0}$ is semisimple. Then any simple object of $A$-mod is pure, and any pure object is semisimple. We will use later

Lemma 2.1.2. Let $A$ be a positively graded ring such that $A_{0}$ is semisimple. Let $M, N \in A$-mod be pure of weights $m, n$. Then $\operatorname{ext}_{A}^{i}(M, N)=0$ for $i>m-n$.

Proof. Without restriction we may assume $m=0$. Now suppose even more generally that $M$ lives only in positive degrees, $M=\bigoplus_{j \geq 0} M_{j}$. We may easily prove by induction that $M$ admits a graded projective resolution

$$
\ldots \rightarrow P^{2} \rightarrow P^{1} \rightarrow P^{0} \rightarrow M
$$

such that $P^{i}$ lives only in degrees $\geq i, P^{i}=\bigoplus_{j \geq i} P_{j}^{i}$. We may use this resolution to compute our ext-groups, and the lemma follows.

Now comes an alternative characterization of Koszul rings.

Proposition 2.1.3. Let $A=\bigoplus_{j \geq 0} A_{j}$ be a positively graded ring and suppose $A_{0}$ is semisimple. The following conditions are equivalent:

(1) A is Koszul.

(2) For any two pure $A$-modules $M, N$ of weights $m, n$ respectively we have $\operatorname{ext}_{A}^{i}(M, N)=0$ unless $i=m-n$.

(3) $\operatorname{ext}_{A}^{i}\left(A_{0}, A_{0}\langle n\rangle\right)=0$ unless $i=n$.

Remark. One may interpret this proposition as saying that a Koszul ring is a positively graded ring that is "as close to semisimple as it can possibly be".

Proof. (1) $\Rightarrow(2)$ : Suppose $A$ is Koszul. Then by definition $A_{0}$ admits a graded projective resolution

$$
\ldots \rightarrow P^{2} \rightarrow P^{1} \rightarrow P^{0} \rightarrow A_{0}
$$

such that $P^{i}=A P_{i}^{i}$.

Now let $M, N$ be as above. By shifting the grading of $M$ and $N$ we may assume $M$ to be pure of weight zero, hence a direct summand of a free $A_{0}$-module, so without restriction we may assume $M=A_{0}$. Then $\operatorname{ext}_{A}^{i}(M, N)$ is the $i$-th cohomology group of the complex $\operatorname{hom}_{A}\left(P^{j}, N\right)$. But since $P^{j}=A P_{j}^{j}$ and $N=N_{-n}$, all terms of this complex other then $\operatorname{hom}_{A}\left(P^{-n}, N\right)$ vanish. Thus $\operatorname{ext}_{A}^{i}(M, N)=0$ unless $i=-n$.

$(2) \Rightarrow(3)$ is trivial.

$(3) \Rightarrow(1)$ : To find the graded projective resolution of $A_{0}$ required in the definition of a Koszul ring we proceed by induction. Certainly $A$ will do as $P^{0}$. Suppose now a resolution

$$
P^{i} \rightarrow \ldots \rightarrow P^{2} \rightarrow P^{1} \rightarrow P^{0} \rightarrow A_{0}
$$

as required has already been constructed up to step $i$, such that $P^{i}$ is projective, $P^{i}=A P_{i}^{i}$ and the differential is injective on $P_{i}^{i}$. Put

$$
K=\operatorname{ker}\left(P^{i} \rightarrow P^{i-1}\right) .
$$

Then for pure $N \operatorname{ext}_{A}^{i+1}\left(A_{0}, N\right)=\operatorname{hom}_{A}(K, N)$ and (3) implies that $\operatorname{hom}_{A}\left(K, A_{0}\langle n\rangle\right)$ $=0$ unless $n=i+1$. But this means precisely that $K=A K_{i+1}$, so we may put $P^{i+1}=A \otimes_{A_{0}} K_{i+1}$ and thus complete the induction step. 
2.2. The opposed ring of a Koszul ring is Koszul. Denote by Mod- $R$ (resp. $\bmod -R$ ) the categories of (resp. graded) right modules over a (graded) ring $R$. Morphisms and extensions in these categories will be denoted by $\mathrm{Hom}_{-R}$, Ext $-R$ and hom ${ }_{-R}$, ext- $R$ respectively. Now let $A, k$ be rings and $X$ an $A$ - $k$-bimodule. Then for $M \in \operatorname{Mod}-A, N \in \operatorname{Mod}-k$ we have a standard isomorphism

$$
\operatorname{Hom}_{-A}\left(M, \operatorname{Hom}_{-k}(X, N)\right)=\operatorname{Hom}_{-k}\left(M \otimes_{A} X, N\right) .
$$

For $V \in \operatorname{Mod}-k$ put ${ }^{*} V=\operatorname{Hom}_{-k}(V, k) \in k$-Mod. Now consider $k$ as a graded ring concentrated in degree zero. Then for $V \in \bmod -k$ define ${ }^{\circledast} V \in k$-mod by $\left({ }^{\circledast} V\right)_{i}={ }^{*}\left(V_{-i}\right)$.

Suppose next that $A$ is a graded ring, $k$ a graded ring concentrated in degree zero, $M \in \bmod -A$ a graded $A$-module and $X$ a graded $A$ - $k$-bimodule. Then ${ }^{\circledast} X$ is a graded $k$ - $A$-bimodule, and it is easy to see that our standard isomorphism above, when applied to $N=k$, induces an isomorphism

$$
\operatorname{hom}_{-A}\left(M,{ }^{\circledast} X\right)={ }^{*}\left(\left(M \otimes_{A} X\right)_{0}\right)
$$

with $\left(M \otimes_{A} X\right)_{0}$ the degree zero component of the graded space $\left(M \otimes_{A} X\right)$. In particular, if $k$ is semisimple and $X$ projective as a left $A$-module, then ${ }^{\circledast} X$ is an injective object of $\bmod -A$. Now we can prove

Proposition 2.2.1. The opposed ring of a Koszul ring is Koszul.

Proof. Let $A$ be our Koszul ring, and put $A_{0}=k$. Consider a resolution

$$
\ldots \rightarrow P^{2} \rightarrow P^{1} \rightarrow P^{0} \rightarrow k
$$

of $k \in A$-mod such that $P^{i}=A P_{i}^{i}$. By the first remark following Definition 1.2.1 of a Koszul ring we know that this is in fact a complex of graded $A$ - $k$-bimodules. The arguments above now show that

$$
k \hookrightarrow{ }^{\circledast} P^{0} \rightarrow{ }^{\circledast} P^{1} \rightarrow{ }^{\circledast} P^{2} \rightarrow \ldots
$$

is an injective resolution of $k$ in $\bmod -A$. Furthermore,

$$
\operatorname{hom}_{-A}\left(k\langle-n\rangle,{ }^{\circledast} P^{i}\right)={ }^{*}\left(\left(k \otimes_{A} P^{i}\right)_{n}\right)={ }^{*}\left(\left(P_{i}^{i}\right)_{n}\right)=0 \text { if } i \neq n .
$$

This in turn shows that $\operatorname{ext}_{-A}^{i}(k\langle-n\rangle, k)=0$ for $i \neq n$, and hence $A^{\text {opp }}$ is Koszul.

2.3. Any Koszul ring is quadratic. Let us fix from now on a semisimple ring $k$ and put $\otimes=\otimes_{k}$. Let $A=\bigoplus_{i \geq 0} A_{i}$ be a positively graded ring, and suppose $A_{0}=k$.

Proposition 2.3.1. The following conditions are equivalent: (1) $\operatorname{ext}_{A}^{1}(k, k\langle n\rangle) \neq 0$ only for $n=1$, and (2) $A$ is generated by $A_{1}$ over $k$.

Proof. Put $I=A_{>0}$ and consider the short exact sequence $I \hookrightarrow A \rightarrow k$. Then

$$
\begin{aligned}
(2) & \Leftrightarrow I=A A_{1} \\
& \Leftrightarrow \operatorname{hom}_{A}(I, k\langle n\rangle)=0 \quad \text { for } n \neq 1 \\
& \Leftrightarrow \operatorname{ext}_{A}^{1}(k, k\langle n\rangle)=0 \text { for } n \neq 1 \\
& \Leftrightarrow(1) . \quad \square
\end{aligned}
$$

Theorem 2.3.2. Suppose $A$ is generated by $A_{1}$ over $k$. If $\operatorname{ext}_{A}^{2}(k, k\langle n\rangle) \neq 0$ implies $n=2$, then $A$ is quadratic. 
Proof. Put $W=\operatorname{ker}(m)$, so that we have an exact sequence

$$
0 \rightarrow W \rightarrow A \otimes A_{1} \rightarrow A \rightarrow k \rightarrow 0 .
$$

Certainly $W \subset I \otimes A_{1}$. Hence

$$
\begin{aligned}
\operatorname{ext}_{A}^{2}(k, k\langle n\rangle) & =\operatorname{hom}_{A}(W, k\langle n\rangle) \\
& =\operatorname{hom}_{k}(W / I \cdot W, k\langle n\rangle) .
\end{aligned}
$$

So our assumption implies that $W / I \cdot W$ is concentrated in degree 2. Now write $A$ as a quotient of $T_{k} A_{1}$, and let $x \in A_{1}^{\otimes n}$ be a relation of $A$ of degree $>2$, not contained in the two-sided ideal of $T_{k} A_{1}$ generated by all relations of smaller degrees. Consider the natural surjections

$$
A_{1}^{\otimes n}=A_{1}^{\otimes n-1} \otimes A_{1} \stackrel{p}{\rightarrow} A_{n-1} \otimes A_{1} \stackrel{m}{\rightarrow} A_{n} .
$$

Certainly $m(p(x))=0$; thus $p(x) \in W$. We claim that $p(x) \notin I \cdot W$. Let $R_{n} \subset A_{1}^{\otimes n}$ be the relations, i.e. the kernel of the multiplication map to $A_{n}$. Certainly $p(x) \in I \cdot W$ implies $p(x) \in A_{1} \cdot W$, hence $p(x)=p(y)$ for suitable $y \in A_{1} \otimes R_{n-1}$. But then $p(x-y)=0$, hence $x-y \in R_{n-1} \otimes A_{1}$. So from $p(x) \in I \cdot W$ it follows that $x \in A_{1} \otimes R_{n-1}+R_{n-1} \otimes A_{1}$, contrary to our assumptions on $x$. Thus indeed $p(x) \notin I \cdot W$. But then $p(x)$ gives an element of degree $>2$ in $W / I \cdot W$, and this contradiction proves the theorem.

Corollary 2.3.3. Any Koszul ring is quadratic.

Proof. This follows from the above proposition and theorem along with the characterization of a Koszul ring given in Proposition 2.1.3.

2.4. Filtrations on modules. Let $M$ be a module over a ring. Then $M$ has a biggest semisimple submodule $\operatorname{soc} M$, the socle of $M$. We put $0=\operatorname{soc}^{0} M$ and define the socle filtration on $M$ inductively by $\operatorname{soc}^{i} M=\pi^{-1} \operatorname{soc}\left(M / \operatorname{soc}^{i-1} M\right)$, where $\pi: M \rightarrow M / \mathrm{soc}^{i-1} M$ is the projection. If $M$ has finite length, then the set of submodules $\{N \subset M \mid M / N$ is semisimple $\}$ contains a smallest element, called the radical $\operatorname{rad} M$ of $M$. We put $M=\operatorname{rad}^{0} M$ and define the radical filtration on $M$ inductively by $\operatorname{rad}^{i} M=\operatorname{rad}\left(\operatorname{rad}^{i-1} M\right)$.

Now let $A$ be a positively graded ring and $M \in A$-mod a graded $A$-module. Then $M$ has a filtration by the submodules $G_{j} M=\bigoplus_{i \geq j} M_{i}$. We call this the "grading filtration". If $A_{0}$ is semisimple, the sucessive subquotients of the grading filtration are semisimple.

Proposition 2.4.1. Let $A$ be a graded ring. Suppose that (1) $A_{0}$ is semisimple, and (2) $A$ is generated by $A_{1}$ over $A_{0}$. Let $M \in A$-mod be a graded A-module of finite length.

If $\operatorname{soc} M$ (resp. $M / \operatorname{rad} M)$ is simple, then the socle (resp. radical) filtration on $M$ coincides with the grading filtration, up to shift.

Proof. Put $I=A_{>0}$. Certainly $I^{n} M=0$ for $n \gg 0$; thus $I N=0$ for any simple subquotient of $M$. We thus have $\operatorname{soc} M=\{m \in M \mid I m=0\}(\operatorname{resp} . \operatorname{rad} M=I M)$ and more generally $\operatorname{soc}^{i} M=\left\{m \in M \mid I^{i} m=0\right\}\left(\right.$ resp. $\left.\operatorname{rad}^{i} M=I^{i} M\right)$. But since

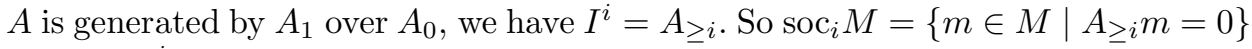
(resp. $\operatorname{rad}^{i} M=A_{\geq i} M$ ).

Suppose now that $M / \operatorname{rad} M$ is simple or, more generally, concentrated in one degree $j$. Then $M=A M_{j}$, and hence $\operatorname{rad}^{i} M=A_{\geq i} M=A_{\geq i} M_{j}=\bigoplus_{n \geq i+j} M_{n}$. Suppose dually that $\operatorname{soc} M$ is simple or, more generally, concentrated in one degree 
$j$. Then for any $m \in M_{j-\nu}, m \neq 0$, there exists $a \in A_{\nu}$ such that $0 \neq a m \in M_{j}$. In other words, $\bigoplus_{n \geq j-i} M_{n}=\left\{m \in M \mid A_{\geq i} m=0\right\}=\operatorname{soc}_{i} M$.

\subsection{Unicity of gradings.}

Proposition 2.5.1. Let $A$ be a positively graded ring such that $A$ is generated by $A_{1}$ over $A_{0}$. Put $I=A_{>0}$. Then we have a canonical isomorphism of graded rings

$$
A \cong \bigoplus_{i} I^{i} / I^{i+1}
$$

Proof. This is clear.

Let $A$ be a ring. By a "Koszul grading on $A$ " we mean a grading such that the corresponding graded ring is Koszul.

Corollary 2.5.2. Let $A$ be an artinian ring. Then any two Koszul gradings on $A$ give rise to isomorphic graded rings.

Proof. They both give rise to graded rings that are canonically isomorphic to the graded ring $\bigoplus_{i}\left(\operatorname{rad}^{i} A / \operatorname{rad}^{i+1} A\right)$.

Let $A$ be a graded ring. We have the functor forgetting the grading

$$
v: A \text {-mod } \rightarrow A \text {-Mod. }
$$

By a "lift" of $M \in A$-Mod we mean an object $\tilde{M} \in A$-mod together with an isomorphism $v \tilde{M} \cong M$.

Lemma 2.5.3. Suppose $M \in A$-Mod is indecomposable and of finite length. Then, if $M$ admits a lift, this lift is unique up to grading shift and isomorphism.

Proof. Since $M$ is indecomposable, its endomorphism ring is local, i.e. the non-automorphisms form an ideal. If $\tilde{M}^{\prime}$ is another lift of $M$, we have $\operatorname{Hom}_{A}(M, M)=$ $\bigoplus_{n} \operatorname{hom}_{A}\left(\tilde{M}^{\prime}, \tilde{M}\langle n\rangle\right)$ and can decompose id $=\sum \mathrm{id}_{n}$. Since id is an automorphism of $M$, one of the $\mathrm{id}_{n}$ has to induce an automorphism of $M$, and one sees easily that this $\operatorname{id}_{n}$ has to be an isomorphism $\tilde{M}^{\prime} \rightarrow \tilde{M}\langle n\rangle$.

2.6. The Koszul complex. Recall our fixed semisimple ring $k$. Let $V$ be a $k$ bimodule, $R \subset V \otimes V$ a sub-bimodule and $A=T_{k} V /(R)$ the corresponding quadratic ring. We want to define its Koszul complex $\ldots \rightarrow K^{2} \rightarrow K^{1} \rightarrow K^{0}$. This is to be a complex in $A$-mod. Let us define the $k$-sub-bimodules

$$
K_{i}^{i}=\bigcap_{\nu} V^{\otimes \nu} \otimes R \otimes V^{\otimes i-\nu-2} \subset V^{\otimes i} .
$$

In particular $K_{0}^{0}=k, K_{1}^{1}=V, K_{2}^{2}=R$. Then put $K^{i}=A \otimes K_{i}^{i}$ (with the grading $K^{i}=\bigoplus K_{j}^{i}$ defined in such a way that $\left.K_{i}^{i}=K_{i}^{i}\right)$. Define the differential $d: K^{i} \rightarrow$ $K^{i-1}$ to be the restriction of $A \otimes V^{\otimes i} \rightarrow A \otimes V^{\otimes i-1}, a \otimes v_{1} \otimes \ldots v_{i} \mapsto a v_{1} \otimes \ldots v_{i}$. Clearly $d^{2}=0$, so here is our Koszul complex. Clearly $K^{0}=A$.

Theorem 2.6.1. Let $A=T_{k} V /(R)$ be a quadratic ring. Then $A$ is Koszul if and only if its Koszul complex is a resolution of $k=A / A_{>0}$.

Proof. If the Koszul complex is a resolution of $k$, then $A$ is Koszul by definition.

On the other hand suppose $A$ is Koszul, so by Proposition 2.1.3 $\operatorname{ext}_{A}^{i}(k, k\langle n\rangle)=0$ if $i \neq n$. Certainly $K^{1} \rightarrow K^{0} \rightarrow k$ is right exact. Thus we need only show that $H^{p}\left(K^{\bullet}\right)=0$ for all $p \geq 1$. We use induction on $p$. Let $Z^{p}=\operatorname{ker}(d) \subset K^{p}$ be the 
submodules of cycles. Since the differential $d$ induces an injection $K_{p}^{p} \hookrightarrow K_{p}^{p-1}$, we find that $Z^{p}$ lives only in degrees $\geq p+1$. Using induction we get

$$
\operatorname{ext}_{A}^{p+1}(k, k\langle n\rangle)=\operatorname{coker}\left\{\operatorname{hom}_{A}\left(K^{p}, k\langle n\rangle\right) \rightarrow \operatorname{hom}_{A}\left(Z^{p}, k\langle n\rangle\right)\right\} .
$$

Hence $\operatorname{ext}_{A}^{p+1}(k, k\langle n\rangle)=\operatorname{hom}_{A}\left(Z^{p}, k\langle n\rangle\right)$, and by Koszulity this doesn't vanish only for $n=p+1$. In particular $Z^{p} \in A$-mod is generated by $Z_{p+1}^{p}$.

But it is easy to see directly that $Z_{p+1}^{p}$ is precisely the image of $K_{p+1}^{p+1}$ under the differential of the Koszul complex. Thus $Z^{p}=\operatorname{im}(d)$ and $H^{p}\left(K^{\bullet}\right)=0$.

Remark. Generalizing the arguments of [Bac82] (see also [BGS88]), one can prove in the above setting that $A$ is Koszul if and only if for all $n \geq 1$ the set $\left\{V^{\otimes n-i-1} \otimes\right.$ $\left.R \otimes V^{\otimes i-1}\right\}_{i=1}^{n-1}$ generates a distributive lattice of submodules of $V^{\otimes n}$.

2.7. Some linear algebra. Let $k$ be a fixed semisimple ring. All the claims below are easily checked on free modules and follow for arbitrary modules. For $V \in k$-Mod define $V^{*}=\operatorname{Hom}_{k}(V, k) \in \operatorname{Mod}-k$ via $(f a)(v)=(f(v)) a \forall f \in V^{*}, v \in V, a \in k$. For $W \in \operatorname{Mod}-k$ define ${ }^{*} W=\operatorname{Hom}_{-k}(W, k) \in k$-Mod via $(a g)(w)=a(g(w)) \forall g \in$ ${ }^{*} W, w \in W, a \in k$. For finitely generated $V, W$ the canonical maps $V \rightarrow{ }^{*}\left(V^{*}\right)$ and $W \rightarrow\left({ }^{*} W\right)^{*}$ given by evaluation are isomorphisms. If $V_{1} \subset V$ (resp. $\left.W_{1} \subset W\right)$ is a submodule, we put $V_{1}^{\perp}=\left\{f \in V^{*} \mid f\left(V_{1}\right)=0\right\}$ (resp. ${ }^{\perp} W_{1}=\left\{g \in{ }^{*} W \mid g\left(W_{1}\right)=\right.$ $0\})$. These are submodules.

For a $k$-bimodule $V \in k$-Mod- $k$ both $V^{*}$ and ${ }^{*} V$ are bimodules defined as follows: Define $(a f)(v)=f(v a) \forall f \in V^{*}, v \in V, a \in k$ and $(g a)(v)=g(a v) \forall g \in{ }^{*} V, v \in$ $V, a \in k$. Both canonical maps $V \rightarrow{ }^{*}\left(V^{*}\right)$ and $V \rightarrow\left({ }^{*} V\right)^{*}$ are now morphisms of bimodules. If $V_{1} \subset V$ is a sub-bimodule, both $V_{1}^{\perp} \subset V^{*}$ and ${ }^{\perp} V_{1} \subset{ }^{*} V$ are sub-bimodules.

Recall that $\otimes=\otimes_{k}$ always. For $V \in k$-Mod- $k, W \in k$-Mod define a map $W^{*} \otimes V^{*} \rightarrow(V \otimes W)^{*}$ by $(g \otimes f)(v \otimes w)=f(v g(w))$. This is an isomorphism for $W=k$, hence for any finitely generated $W \in k$-Mod. If $W \in k$-Mod- $k$ this is actually a morphism of $k$-bimodules. More generally, if $V_{1}, \ldots, V_{n} \in k$-Mod- $k$ are finitely generated as left $k$-modules, then canonically $V_{1}^{*} \otimes \ldots \otimes V_{n}^{*}=\left(V_{n} \otimes \ldots \otimes V_{1}\right)^{*}$ as $k$-bimodules, where the isomorphism is given by $\left(f_{1} \otimes \ldots \otimes f_{n}\right)\left(v_{n} \otimes \ldots \otimes v_{1}\right)=$ $f_{n}\left(v_{n} f_{n-1}\left(v_{n-1} \ldots f_{1}\left(v_{1}\right) \ldots\right)\right)$.

Dually, for $V \in \operatorname{Mod}-k, W \in k$-Mod- $k$ define a map ${ }^{*} W \otimes{ }^{*} V \rightarrow{ }^{*}(V \otimes W)$ by $(g \otimes f)(v \otimes w)=g(f(v) w)$. This is an isomorphism for finitely generated $V \in$ Mod- $k$. If $V \in k$-Mod- $k$, the above morphism is actually a morphism of $k$-bimodules. Again if $V_{1}, \ldots, V_{n} \in k$-Mod- $k$ are finitely generated as right $k$-modules, then ${ }^{*} V_{1} \otimes \ldots \otimes{ }^{*} V_{n}={ }^{*}\left(V_{n} \otimes \ldots \otimes V_{1}\right)$ as $k$-bimodules, where $\left(f_{1} \otimes \ldots \otimes f_{n}\right)\left(v_{n} \otimes \ldots \otimes v_{1}\right)=$ $f_{1}\left(\ldots f_{n-1}\left(f_{n}\left(v_{n}\right) v_{n-1}\right) \ldots v_{1}\right)$.

For $V, W \in k$-Mod define a map $V^{*} \otimes W \rightarrow \operatorname{Hom}_{k}(V, W)$ by $(f \otimes w)(v)=f(v) w$. This is an isomorphism if $V$ or $W$ is finitely generated. If $V$ (resp. $W$ ) is a $k$ bimodule, then our map is compatible with the resulting left (resp. right) $k$-actions on both spaces. For $V, W \in \operatorname{Mod}-k$ define a map $W \otimes{ }^{*} V \rightarrow \operatorname{Hom}_{-k}(V, W)$ by $(w \otimes f)(v)=w f(v)$. This is an isomorphism if $V$ or $W$ is finitely generated. If $V$ (resp. $W$ ) is a $k$-bimodule, then our map is compatible with the resulting right (resp. left) $k$-actions on both spaces.

Suppose $V \in k$-Mod- $k$. Then there are canonical bimodule homomorphisms $V \otimes$ $V^{*} \rightarrow k$ and ${ }^{*} V \otimes V \rightarrow k$ given by $v \otimes f \mapsto f(v)$ and $g \otimes v \mapsto g(v)$ respectively. Dually there are canonical bimodule homomorphisms $k \rightarrow V^{*} \otimes V=\operatorname{Hom}_{k}(V, V)$ 
and $k \rightarrow V \otimes{ }^{*} V=\operatorname{Hom}_{-k}(V, V)$, where $a \in k$ is mapped to right (resp. left) multiplication on $V$.

2.8. The quadratic dual rings and the Koszul complex. Call a graded ring $A$ with $A_{0}=k$ a graded ring "over $k$ ". We begin by defining the quadratic dual rings. Recall Definition 1.2.4 of a left (resp. right) finite graded ring.

Definition 2.8.1. Let $A$ be a left finite quadratic ring over $k$, say $A=T_{k} V /(R)$. Then its left dual $A^{!}$is the right finite quadratic ring over $k$ given by $A^{!}=$ $T_{k}\left(V^{*}\right) /\left(R^{\perp}\right)$ with $R^{\perp} \subset V^{*} \otimes V^{*}=(V \otimes V)^{*}$.

Analogously if $A$ is a right finite quadratic ring over $k$, say $A=T_{k} V /(R)$, then its right dual ! $A$ is the left finite quadratic ring over $k$ given by ${ }^{!} A=T_{k}\left({ }^{*} V\right) /\left({ }^{\perp} R\right)$ with ${ }^{\perp} R \subset{ }^{*} V \otimes{ }^{*} V={ }^{*}(V \otimes V)$.

Remark. Obviously ${ }^{!}\left(A^{!}\right)=A$ and $\left({ }^{!} A\right)^{!}=A$ for a left, resp. right finite quadratic $\operatorname{ring} A$.

Now let $A=T_{k} V /(R)$ be a left finite quadratic ring over $k$. Then its Koszul complex is (isomorphic to) the complex

$$
\ldots \rightarrow A \otimes{ }^{*}\left(A_{2}^{!}\right) \rightarrow A \otimes *\left(A_{1}^{!}\right) \rightarrow A,
$$

where we abreviate $\left(A^{!}\right)_{i}=A_{i}^{!}$. The differentials $d: A \otimes \otimes^{*}\left(A_{i+1}^{!}\right) \rightarrow A \otimes *\left(A_{i}^{!}\right)$ may be described as follows: Observe that $A \otimes{ }^{*}\left(A_{i}^{!}\right)=\operatorname{Hom}_{-k}\left(A_{i}^{!}, A\right)$. Under the canonical isomorphism $\operatorname{Hom}_{k}(V, V)=V^{*} \otimes V$ let $\operatorname{id}_{V}=\sum \check{v}_{\alpha} \otimes v_{\alpha}$. Then $(d f)(a)=$ $\sum f\left(a \check{v}_{\alpha}\right) v_{\alpha}$ for $f \in \operatorname{Hom}_{-k}\left(A_{i+1}^{!}, A\right), a \in A_{i}^{!}$. We may also write this without coordinates. Namely, use the obvious maps $A \otimes V \rightarrow A$ and $A_{i}^{!}=A_{i}^{!} \otimes k \rightarrow A_{i}^{!} \otimes$ $V^{*} \otimes V \rightarrow A_{i+1}^{!} \otimes V$ to give the differential as the composition $\operatorname{Hom}_{-k}\left(A_{i+1}^{!}, A\right) \rightarrow$ $\operatorname{Hom}_{-k}\left(A_{i+1}^{!} \otimes V, A \otimes V\right) \rightarrow \operatorname{Hom}_{-k}\left(A_{i}^{!}, A\right)$. To see that this is really our Koszul complex from section 2.6, write

$$
A_{i}^{!}=\left(V^{*}\right)^{\otimes i} / \sum_{\nu}\left(V^{*}\right)^{\otimes i-\nu-2} \otimes R^{\perp} \otimes\left(V^{*}\right)^{\otimes \nu}
$$

We deduce that

$$
*\left(A_{i}^{!}\right)=\bigcap_{\nu} V^{\otimes \nu} \otimes R \otimes V^{\otimes i-\nu-2}=K_{i}^{i} \subset V^{\otimes i} .
$$

Thus $A \otimes{ }^{*}\left(A_{i}^{!}\right)=K^{i}$, and the reader may check that the differentials correspond.

\subsection{The quadratic dual of a Koszul ring is Koszul.}

Proposition 2.9.1. Let $A$ be a left finite Koszul ring. Then $A^{!}$is Koszul as well.

Proof. The total space of the Koszul complex $A \otimes{ }^{\circledast} A^{!}$is an $A$ - $A^{!}$-bimodule, and the differential commutes with the action of both $A$ and $A$ ! . In fact we have a bigraded space

$$
A \otimes{ }^{\circledast} A^{!}=\bigoplus_{i, j} A_{i} \otimes^{*} A_{-j}^{!}
$$

concentrated in the lower right quadrant, the differential has bidegree $(1,1)$, and the only cohomology appears in bidegree $(0,0)$.

If we apply $\operatorname{Hom}_{k}(, k)$ to every component of this bigraded space, we obtain a bigraded space

$$
A^{!} \otimes A^{\circledast}=\bigoplus_{i, j} A_{j}^{!} \otimes A_{-i}^{*}
$$


concentrated in the upper left quadrant with differential of bidegree $(1,1)$ commuting with the left $A^{!}$-action, and again the only cohomology appears in bidegree $(0,0)$. But we may also read this as a graded projective resolution

$$
\ldots \rightarrow A^{!} \otimes A_{2}^{*} \rightarrow A^{!} \otimes A_{1}^{*} \rightarrow A^{!} \otimes A_{0}^{*} \rightarrow k
$$

of $k \in A^{!}$-mod, and this then shows that $A^{!}$is Koszul.

2.10. The quadratic dual and cohomology. Let $R$ be a ring and $M \in R$-Mod. We will repeat the definition of $\operatorname{Ext}_{R}^{\bullet}(M, M)$ in a form suitable for our purposes. For two complexes $P^{\bullet}, Q^{\bullet}$ of $R$-modules consider the graded space $\mathcal{H o m}_{R}^{\bullet}\left(P^{\bullet}, Q^{\bullet}\right)$ with

$$
\mathcal{H o m}_{R}^{i}\left(P^{\bullet}, Q^{\bullet}\right)=\bigoplus_{n} \operatorname{Hom}_{R}\left(P^{n}, Q^{n-i}\right) .
$$

The differentials $d$ of degree $(-1)$ on $P^{\bullet}$ and $Q^{\bullet}$ give rise to a differential of degree $(+1)$ on $\mathcal{H o m}_{R}^{\bullet}\left(P^{\bullet}, Q^{\bullet}\right)$ by $d f=d \circ f-(-1)^{|f|} f \circ d$ for $f \in \mathcal{H o m}_{R}^{|f|}\left(P^{\bullet}, Q^{\bullet}\right)$. For $f, g \in \mathcal{E} n d_{R}^{\bullet}\left(P^{\bullet}\right)$ we have the Leibniz rule $d(f g)=(d f) g+(-1)^{|f|} f(d g)$. Thus the cohomology $H^{\bullet} \mathcal{E} n d_{R}^{\bullet}\left(P^{\bullet}\right)$ has the structure of a graded ring. If now $P^{\bullet} \rightarrow M$ is a projective resolution of $M \in R$-Mod, then more or less by definition

$$
H^{\bullet} \mathcal{E} n d_{R}^{\bullet}\left(P^{\bullet}\right)=\operatorname{Ext}_{R}^{\bullet}(M, M) .
$$

Theorem 2.10.1. Let $A$ be a left finite Koszul ring over $k$. Then $\operatorname{Ext}_{A}^{\bullet}(k, k)=$ $\left(A^{!}\right)^{\mathrm{opp}}$ canonically.

Proof. Consider the Koszul complex $\left(K^{\bullet}, d\right)$ and form the complex $\left(\tilde{K}^{\bullet}, \tilde{d}\right)$ with $\tilde{K}^{i}=K^{i}, \tilde{d}=(-1)^{i} d: K^{i} \rightarrow K^{i-1}$. Then $\tilde{K}^{\bullet}$ is also a projective resolution of $k$. The right action of $A^{!}$on $K^{\bullet}=A \otimes{ }^{\circledast} A^{!}$gives us a homomorphism of graded rings

$$
\left(A^{!}\right)^{\text {opp }} \rightarrow \mathcal{E} n d_{A}^{\bullet}\left(K^{\bullet}\right) \text {. }
$$

Since this right action commutes with the differential $d$ of the Koszul complex, the image of

$$
\left(A^{!}\right)^{\text {opp }} \rightarrow \mathcal{E} n d_{A}^{\bullet}\left(\tilde{K}^{\bullet}\right)
$$

even consists of cycles. (This is the reason we work with $\tilde{K}^{\bullet}$ and not with $K^{\bullet}$.) Hence our morphism above induces a morphism

$$
\left(A^{!}\right)^{\mathrm{opp}} \rightarrow H^{\bullet} \mathcal{E} n d_{A}^{\bullet}\left(\tilde{K}^{\bullet}\right)
$$

and we just have to show that this is an isomorphism. For this, consider the composition

$$
\left(A^{!}\right)^{\text {opp }} \rightarrow \mathcal{E} n d_{A}^{\bullet}\left(\tilde{K}^{\bullet}\right) \rightarrow \mathcal{H o m}_{A}^{\bullet}\left(\tilde{K}^{\bullet}, k\right)
$$

where $k$ is considered as a complex concentrated in degree zero. We consider $\left(A^{!}\right)^{\text {opp }}$ as a complex with zero differential, and then both these maps are chain maps. The second map certainly induces an isomorphism on cohomology, and the composition is just the identity from $A^{!}$to

$$
\mathcal{H o m}_{A}^{\bullet}\left(\tilde{K}^{\bullet}, k\right)=\bigoplus_{i} \operatorname{Hom}_{A}\left(A \otimes{ }^{*}\left(A_{i}^{!}\right), k\right)=A^{!} .
$$

Hence the first map has to induce an isomorphism on cohomology as well.

We can now prove Theorem 1.2.5 from the introduction.

Theorem 2.10.2. Suppose $A$ is a left finite Koszul ring. Then $E(A)$ is also a left finite Koszul ring, and $E(E(A))=A$ canonically. 
Proof. $E(A)=\left(A^{!}\right)^{\text {opp }}$ is obviously left finite, and is Koszul by Propositions 2.2.1 and 2.9.1. Then $E(E(A))=\left(\left(\left(A^{!}\right)^{\text {opp }}\right)^{!}\right)^{\text {opp }}={ }^{!}\left(A^{!}\right)=A$.

2.11. A numerical Koszulity criterion. Fix a field $F$ and suppose that (1) the positively graded ring $A$ is an $F$-algebra, i.e. there is a central embedding $F \subset A$. Suppose in addition that (2) $\operatorname{dim}_{F} A_{i}<\infty$ for all $i$, and (3) $A_{0}=k=\bigoplus_{x \in \mathcal{W}} F 1_{x}$ for pairwise orthogonal idempotents $1_{x}$ indexed by a finite set $\mathcal{W}$, so in particular $k^{\text {opp }}=k$.

Then we may form the Hilbert polynomial $P(A, t)$ of $A$, a $\mathcal{W} \times \mathcal{W}$-matrix with entries in power series $\mathbb{Z}[[t]]$ given by

$$
P(A, t)_{x, y}=\sum t^{i} \operatorname{dim}\left(1_{x} A_{i} 1_{y}\right)
$$

To simplify notation, set $\operatorname{dim}\left(1_{x} M 1_{y}\right)=[M]_{x, y}$ for any $M \in k \otimes_{F} k$-Mod $\subset$ $k$-Mod- $k$. The following lemma motivates our criterion.

Lemma 2.11.1. Suppose a positively graded $F$-algebra $A$ satisfying conditions (1)(3) above is Koszul. Then the matrix equation $P(A, t) P\left(A^{!},-t\right)^{T}=1$ holds.

Remark. We do not know whether for a quadratic ring $A$ as in the proposition the matrix equation $P(A, t) P\left(A^{!},-t\right)^{T}=1$ implies that $A$ is Koszul.

Proof. The Euler-Poincaré principle for the Koszul complex yields

$$
\sum_{i, j}(-1)^{i} t^{i+j}\left[A_{i} \otimes *\left(A_{j}^{!}\right)\right]_{x, y}=\left[A_{0}\right]_{x, y} .
$$

Now the result follows from the identities $\left[A_{0}\right]_{x, y}=\delta_{x, y},\left[{ }^{*} M\right]_{x, y}=[M]_{y, x}$ and $[M \otimes N]_{x, y}=\sum_{z}[M]_{x, z}[N]_{z, x}$. (Recall that $\otimes=\otimes_{k}$ always!)

Now forget the grading on $A$ and form the positively graded $F$-algebra $E=$ $E(A)=\operatorname{Ext}_{A}^{\bullet}(k, k)$. Certainly $E_{0}=k^{\text {opp }}=k$, and if $A$ is left noetherian then $\operatorname{dim} E_{i}<\infty$ for all $i$, so we can form the Hilbert polynomial $P(E, t)$ of $E$.

Theorem 2.11.1. Suppose $A$ is a left noetherian $F$-algebra satisfying the conditions (1)-(3) above. Form the graded F-algebra $E=E(A)$. Then the following conditions are equivalent:

(i) A is Koszul.

(ii) The matrix equation $P(A, t) P(E,-t)=1$ holds.

Remark. Löfwall [Löf86] calls (ii) a "Fröberg condition".

Proof. (i) $\Rightarrow$ (ii). If $A$ is Koszul we know by Theorem 2.10 .1 that $E \cong\left(A^{!}\right)^{\text {opp }}$. Now apply the lemma.

(ii) $\Rightarrow$ (i). Certainly $k$ is a graded $A$-module and a graded right $k$-module, and these two actions commute and induce the same $F$-action. In other words, $k \in A \otimes_{F} k$-mod. We are going to construct inductively a resolution

$$
\ldots \rightarrow P^{2} \rightarrow P^{1} \rightarrow P^{0} \rightarrow k
$$

of $k$ in $A \otimes_{F} k$-mod as follows: Take $P^{0}=A$. If $P^{i}$ is already constructed, put $K^{i}=\operatorname{ker}\left(P^{i} \rightarrow P^{i-1}\right)$ and choose a complement $V^{i}$ in $k \otimes_{F} k-\bmod$ of $A_{>0} \cdot K^{i} \subset K^{i}$. So $V^{i}$ is a graded $k$-bimodule $V^{i}=\bigoplus V_{j}^{i}$, and we put $P^{i+1}=A \otimes V^{i}$. Take $V^{0}=k$ for convenience. So we get a resolution of $k$ of the form

$$
\ldots \rightarrow A \otimes V^{2} \rightarrow A \otimes V^{1} \rightarrow A \otimes V^{0} \rightarrow k
$$


with the $V^{i}$ some graded $k$-bimodules $V^{i}=\bigoplus V_{j}^{i}$ such that all differentials of the resolution are compatible with the right $k$-action. By construction $P^{i+1}$ gets mapped to $A_{>0} \cdot P^{i}$, hence the differential on the complex of the $\operatorname{Hom}_{A}\left(P^{\bullet}, k\right)$ vanishes. Also by construction all $P^{i}$ are projective in $A$-mod, hence $\operatorname{Ext}_{A}^{i}(k, k)=$ $\left(V^{i}\right)^{*}, \operatorname{ext}_{A}^{i}(k, k\langle j\rangle)=\left(V_{j}^{i}\right)^{*}$, and the reader may check that even $\left[E_{i}\right]_{x, y}=\left[V^{i}\right]_{x, y}$.

We form the matrix of power series $P(V, t)$ with $P(V, t)_{x, y}=\sum\left[V^{i}\right]_{x, y} t^{i}$ and deduce from (ii) the equation $P(A, t) P(V,-t)=1$. Thus, in the Grothendieck group of a suitable category of $k$-bimodules,

$$
\sum_{i=0}^{n}(-1)^{i} A_{i} \otimes V^{n-i}=0
$$

for $n \geq 1$. To prove that $A$ is Koszul we have to check that $V_{j}^{n}=0$ for all $n \geq 1, j \neq$ $n$. From Lemma 2.1.2 we deduce that $\operatorname{ext}_{A}^{i}(k, k\langle j\rangle)=0$ for $j<i$. Thus $V_{j}^{i}=0$ for $j<i$. We need $V_{j}^{i}=0$ for $j \neq i$.

We proceed by induction on $i$. For $i=0$ the statement is clear. Now take the degree $n$ component of the above resolution. Clearly $\left(A \otimes V^{i}\right)_{n}=\bigoplus_{j} A_{n-j} \otimes V_{j}^{i}$. By induction we know that $V^{i}=V_{i}^{i}$ for $i<n$, hence $\left(A \otimes V^{i}\right)_{n}=A_{n-i} \otimes V_{i}^{i}=A_{n-i} \otimes V^{i}$ for $i<n$. By Lemma 2.1.2 (or by construction) we know that $V_{j}^{n}=0$ for $j<n$, hence $\left(A \otimes V^{n}\right)_{n}=A_{0} \otimes V_{n}^{n}$. We find an exact sequence

$$
0 \rightarrow A_{0} \otimes V_{n}^{n} \rightarrow \ldots \rightarrow A_{n-1} \otimes V^{1} \rightarrow A_{n} \otimes V^{0} \rightarrow 0
$$

Thus we get an equation

$$
\sum_{i=0}^{n-1}(-1)^{i} A_{i} \otimes V^{n-i}+(-1)^{n} A_{0} \otimes V_{n}^{n}=0
$$

in the same Grothendieck group as above. Comparing the two equations, we find that $V_{n}^{n}=V^{n}$, and the induction step is complete.

2.12. Koszul duality. Let $B=\bigoplus_{j \geq 0} B_{j}$ be a positively graded ring. Write $C(B)$ for the homotopy category of complexes in $B$-mod. An object $M \in C(B)$ is a complex $\ldots \rightarrow M^{i} \stackrel{\partial}{\rightarrow} M^{i+1} \rightarrow \ldots$ of graded $B$-modules $M^{i}=\bigoplus_{j} M_{j}^{i}$. Let $C^{\uparrow}(B)$ (resp. $C^{\downarrow}(B)$ ) be the full subcategories of $C(B)$ whose objects $M=\bigoplus M_{j}^{i}$ satisfy the conditions

$$
M_{j}^{i}=0 \text { if }\left\{\begin{array}{l}
i \gg 0 \text { or } i+j \ll 0 \text { for } C^{\uparrow}(B), \\
i \ll 0 \text { or } i+j \gg 0 \text { for } C^{\downarrow}(B) .
\end{array}\right.
$$

Thus all nonzero components $M_{j}^{i}$ of an object of $C^{\uparrow}(B)$ (resp. $C^{\downarrow}(B)$ ) are concentrated in a region as indicated in the picture. 


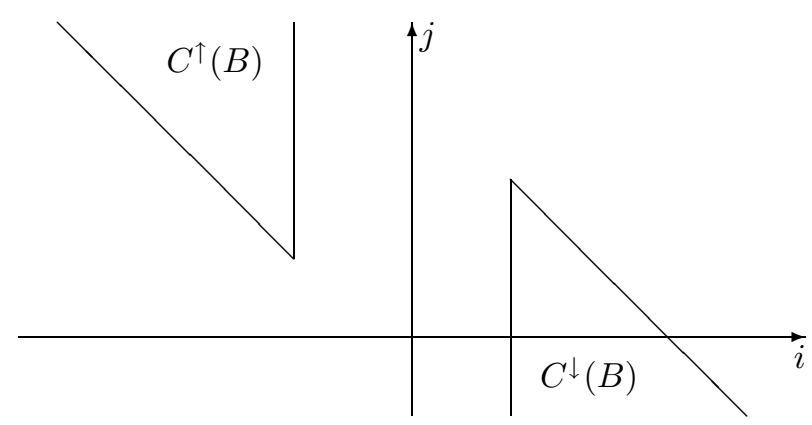

Let $D^{\uparrow}(B), D^{\downarrow}(B)$ denote the localisations of $C^{\uparrow}(B), C^{\downarrow}(B)$ at quasi-isomorphisms. These are also triangulated categories. We want to prove

Theorem 2.12.1. Let $A$ be a left finite Koszul ring. Then there exists an equivalence of triangulated categories

$$
D^{\downarrow}(A) \cong D^{\uparrow}\left(A^{!}\right) \text {. }
$$

Proof [Theorem]. The existence of this equivalence could be deduced from [Kel92]. We explicitly construct such an equivalence. This will be done in several steps. Assume $A_{0}=k$.

Step 1. Construction of a functor $D F: D^{\downarrow}(A) \rightarrow D^{\uparrow}\left(A^{!}\right)$.

For $M \in C(A)$ consider the bigraded $k$-module

$$
F M=A^{!} \otimes M=\bigoplus_{l, i} A_{l}^{!} \otimes M^{i}=\bigoplus_{l, i} \operatorname{Hom}_{A}\left(A \otimes *\left(A_{l}^{!}\right), M^{i}\right)
$$

with anticommuting differentials coming, up to signs, from the differentials of the Koszul complex and of the complex $M$ respectively, and given by

$$
\begin{aligned}
d^{\prime}(a \otimes m) & =(-1)^{i+j} \sum a \check{v}_{\alpha} \otimes v_{\alpha} m \\
d^{\prime \prime}(a \otimes m) & =a \otimes \partial m
\end{aligned}
$$

for $a \in A_{l}^{!}, m \in M_{j}^{i}$. Here $\check{v}_{\alpha}$ and $v_{\alpha}$ are as in 2.8. Consider on $F M$ the total differential $d=d^{\prime}+d^{\prime \prime}$. We can interpret $F M$ as an object of $C\left(A^{!}\right)$, letting

$$
(F M)_{q}^{p}=\bigoplus_{p=i+j, q=l-j} A_{l}^{!} \otimes M_{j}^{i} .
$$

It is easy to check that $M \in C^{\downarrow}(A)$ implies $F M \in C^{\uparrow}\left(A^{!}\right)$. Furthermore, for $M \in C^{\downarrow}(A)$ the spectral sequence with first term $H^{\bullet}\left(F M, d^{\prime \prime}\right)$ associated to the bicomplex $\left(F M ; d^{\prime}, d^{\prime \prime}\right)$ converges to the cohomology $H^{\bullet}(F M, d)$, i.e. $M \in C^{\downarrow}(A)$ acyclic implies $F M \in C^{\uparrow}\left(A^{!}\right)$acyclic. Hence $F$ induces a functor $D F: D^{\downarrow}(A) \rightarrow$ $D^{\uparrow}\left(A^{!}\right)$.

Step 2. Construction of a functor $D G: D^{\uparrow}\left(A^{!}\right) \rightarrow D^{\downarrow}(A)$.

For $N \in C\left(A^{!}\right)$consider the bigraded $k$-module $G N$ given by

$$
(G N)_{l, i}=\operatorname{Hom}_{k}\left(A_{-l}, N^{i}\right)
$$


with anticommuting differentials $d^{\prime}, d^{\prime \prime}$ given by

$$
\begin{aligned}
& \left(d^{\prime} f\right)(a)=(-1)^{i} \sum \check{v}_{\alpha} f\left(v_{\alpha} a\right), \\
& \left(d^{\prime \prime} f\right)(a)=\partial(f(a))
\end{aligned}
$$

for $f \in \operatorname{Hom}_{k}\left(A_{-l}, N^{i}\right)$. The reader may check that $d^{\prime}$ is well defined and is a differential. Consider on $G N$ the total differential $d=d^{\prime}+d^{\prime \prime}$. We can interpret $G N$ as an object of $C(A)$, letting

$$
(G N)_{q}^{p}=\bigoplus_{p=i+j, q=l-j} \operatorname{Hom}_{k}\left(A_{-l}, N_{j}^{i}\right) .
$$

It is easy to check that $N \in C^{\uparrow}\left(A^{!}\right)$implies $G N \in C^{\downarrow}(A)$. Furthermore, for $N \in$ $C^{\uparrow}\left(A^{!}\right)$the spectral sequence with first term $H^{\bullet}\left(G N, d^{\prime \prime}\right)$ associated to the bicomplex $\left(G N ; d^{\prime}, d^{\prime \prime}\right)$ converges to the cohomology $H^{\bullet}(G N, d)$, i.e. $N \in C^{\uparrow}\left(A^{!}\right)$acyclic implies $G N \in C^{\downarrow}(A)$ acyclic. Hence $G$ induces a functor $D G: D^{\uparrow}\left(A^{!}\right) \rightarrow D^{\downarrow}(A)$.

Step 3. Adjointness of our functors.

Consider the functors $F: C^{\downarrow}(A) \rightarrow C^{\uparrow}\left(A^{!}\right)$and $G: C^{\uparrow}\left(A^{!}\right) \rightarrow C^{\downarrow}(A)$. We define an adjointness $(F, G)$. In general, for any $M \in A$-Mod, $N \in A^{!}$-Mod we have canonically

$$
\operatorname{Hom}_{A^{!}}\left(A^{!} \otimes M, N\right)=\operatorname{Hom}_{k}(M, N)=\operatorname{Hom}_{A}\left(M, \operatorname{Hom}_{k}(A, N)\right) .
$$

If for all $m \in M$ there exists $l$ such that $A_{i} m=0$ for $i \geq l$, then even

$$
\operatorname{Hom}_{A^{!}}\left(A^{!} \otimes M, N\right)=\operatorname{Hom}_{k}(M, N)=\operatorname{Hom}_{A}\left(M, \bigoplus_{l} \operatorname{Hom}_{k}\left(A_{l}, N\right)\right) .
$$

Lemma 2.12.2. This canonical isomorphism establishes an adjointness $(F, G)$ of functors between $C^{\downarrow}(A)$ and $C^{\uparrow}\left(A^{!}\right)$.

Proof. Choose $M \in C^{\downarrow}(A), N \in C^{\uparrow}\left(A^{!}\right)$. Choose corresponding homomorphisms $\tilde{f}, f, \hat{f}$ in $\operatorname{Hom}_{A^{!}}(F M, N)=\operatorname{Hom}_{k}(M, N)=\operatorname{Hom}_{A}(M, G N)$. It is easy to see that $\tilde{f}\left((F M)_{j}^{i}\right) \subset N_{j}^{i}$ for all $i, j$ if and only if $\hat{f}\left(M_{q}^{p}\right) \subset(G N)_{q}^{p}$ for all $p, q$. Suppose from now on that $\tilde{f}$ satisfies this condition. We just have to show that $\tilde{f}$ commutes with the differentials if and only if $\hat{f}$ does. But both $\tilde{f}$ and $\hat{f}$ commute with the differentials if and only if

$$
(-1)^{i+j} \sum \check{v}_{\alpha} f\left(v_{\alpha} m\right)+f(\partial m)-\partial(f(m))=0 .
$$

This proves the lemma. Let us perform the calculation for $\hat{f}$. Suppose $m \in M_{j}^{i}$. Then $d(\hat{f}(m))=\hat{f}(\partial m)$ if and only if they have the same value at $1 \in A$, i.e. if and only if $(d(\hat{f}(m)))(1)=(\hat{f}(\partial m))(1)$. By definition $(\hat{f}(\partial m))(1)=f(\partial m)$. On the other hand, $\hat{f}(m)=\sum_{l} \hat{f}_{l}(m)$ with $\hat{f}_{l}(m) \in \operatorname{Hom}_{k}\left(A_{l}, N_{-l-j}^{i+l+j}\right)$. Then

$$
\begin{aligned}
(d(\hat{f}(m)))(1) & =\sum_{l}\left(d\left(\hat{f}_{l}(m)\right)\right)(1) \\
& =\partial\left(\left(\hat{f}_{0}(m)\right)(1)\right)-(-1)^{i+j} \sum \check{v}_{\alpha}\left(\left(\hat{f}_{1}(m)\right)\left(v_{\alpha}\right)\right) \\
& =\partial(f(m))-(-1)^{i+j} \sum \check{v}_{\alpha} f\left(v_{\alpha} m\right) .
\end{aligned}
$$

Thus indeed $\hat{f}$ commutes with the differentials if and only if the above equation on $f$ holds.

Step 4 . We construct an equivalence $D F \circ D G \rightarrow$ id. 
More precisely, we prove

Lemma 2.12.3. For any $N \in C^{\uparrow}\left(A^{!}\right)$the canonical surjection $(F \circ G)(N) \rightarrow N$ from the adjointness is a quasi-isomorphism.

Proof. Consider the splitting over $k$ of our surjection, given by

$$
N=k \otimes \operatorname{Hom}_{k}(k, N) \rightarrow \bigoplus_{l} A^{!} \otimes \operatorname{Hom}_{k}\left(A_{l}, N\right) .
$$

It is sufficient to show that this is a quasi-isomorphism. Consider $N$ as a bicomplex $\left(N_{p, q} ; d^{\prime}, d^{\prime \prime}\right)$ with $N_{0, q}=\bigoplus_{i+j=q} N_{j}^{i}, N_{p, q}=0$ if $p \neq 0, d^{\prime}=0, d^{\prime \prime}=\partial$. Recall from Step 1 how $F(G N)$ is a bicomplex with $(F(G N))_{p, q}=A_{p}^{!} \otimes(G N)^{q}$. In fact our splitting map is a morphism of bicomplexes. Since the spectral sequences in question converge, we need only show that our splitting induces isomorphisms

$$
H^{\bullet}\left(N, d^{\prime}\right) \rightarrow H^{\bullet}\left(F(G N), d^{\prime}\right)
$$

But $F(G N)=\bigoplus \operatorname{Hom}_{k}\left(A_{l} \otimes{ }^{*}\left(A_{p}^{!}\right), N_{j}^{i}\right)$, and the differential $d^{\prime}$ can be described as $d^{\prime} f=(-1)^{i+l}\left(f \circ d_{K}\right)$, where $d_{K}: A_{l} \otimes *\left(A_{p+1}^{!}\right) \rightarrow A_{l} \otimes *\left(A_{p}^{!}\right)$is the differential of the Koszul complex. Now the fact that the Koszul complex is a resolution of $k$ implies that indeed our splitting induces isomorphisms on $H^{\bullet}\left(, d^{\prime}\right)$.

Step 5 . We construct an equivalence id $\rightarrow D G \circ D F$.

More precisely, we prove

Lemma 2.12.4. For any $M \in C^{\downarrow}(A)$ the canonical injection $M \rightarrow(G \circ F)(M)$ from the adjointness is a quasi-isomorphism.

Proof. Consider the splitting $G(F M) \rightarrow M$ over $k$ of our canonical injection given by

$$
\bigoplus \operatorname{Hom}_{k}\left(A_{l}, A^{!} \otimes M\right) \rightarrow \operatorname{Hom}_{k}(k, k \otimes M)=M
$$

It is sufficient to show that this is a quasi-isomorphism. Consider $M$ as a bicomplex $\left(M_{p, q} ; d^{\prime}, d^{\prime \prime}\right)$ with $M_{0, q}=\bigoplus_{i+j=q} M_{j}^{i}, M_{p, q}=0$ if $p \neq 0, d^{\prime}=0, d^{\prime \prime}=\partial$. Recall from Step 2 how $G(F M)$ is a bicomplex with $(G(F M))_{p, q}=\operatorname{Hom}_{k}\left(A_{-p},(F N)^{q}\right)$. In fact our splitting map is a morphism of bicomplexes. Since the spectral sequences in question converge, we need only show that our splitting induces isomorphisms

$$
H^{\bullet}\left(G(F M), d^{\prime}\right) \rightarrow H^{\bullet}\left(M, d^{\prime}\right) .
$$

But $G(F M)=\bigoplus \operatorname{Hom}_{k}\left(A_{l}, A^{!} \otimes M\right)=\bigoplus\left(A_{l}^{*} \otimes A^{!}\right) \otimes M$, and the differential $d^{\prime}$ can be described as being up to signs the differential of the right Koszul complex of $A^{!}$tensor the identity on $M$. Now the fact that the Koszul complex is a resolution of $k$ implies that indeed the above maps on cohomology are isomorphisms.

The theorem is now proven.

As usual, for a complex $X=\left(X^{i}, \partial\right)$ denote by $X[n]$ the shifted complex $(X[n])^{i}=X^{i+n}$ with differentials $(-1)^{n} \partial$. For a graded ring $B$ put $B$-mod ${ }^{\uparrow}=$ $\left\{M \in B\right.$-mod $\mid M_{j}=0$ for $\left.j \ll 0\right\}$ and $B-\bmod ^{\downarrow}=\left\{M \in B\right.$-mod $\mid M_{j}=0$ for $j \gg 0\}$ We will consider these categories as full subcategories $B$-mod $\bmod ^{\uparrow} \subset D^{\uparrow}(B)$, $B$-mod ${ }^{\downarrow} \subset D^{\downarrow}(B)$ of complexes concentrated in degree zero.

Suppose $B_{0}=k$ is semisimple. Then any simple object of $B-\bmod$ is up to a grading shift isomorphic to $B_{0} p$ for some $p \in k$. For any $p \in k$ the surjection $B p \rightarrow B_{0} p$ is a projective cover in $B$-mod ${ }^{\uparrow}$ (and $B$-mod, $B$-Mod.) Define the 
"continuous dual" $B^{\circledast}=\bigoplus_{l} B_{l}^{*} \in B$-Mod- $k$. Then $B_{0} p \hookrightarrow B^{\circledast} p$ is an injective hull in $B$-mod ${ }^{\downarrow}$ for all $p \in k$.

Let us from now on denote by

$$
K: D^{\downarrow}(A) \rightarrow D^{\uparrow}\left(A^{!}\right)
$$

the "Koszul duality functor" $K=D F$ constructed in step 1.

Theorem 2.12.5. Let $A$ be a left finite Koszul ring over $k$.

(i) The functor $K: D^{\downarrow}(A) \rightarrow D^{\uparrow}\left(A^{!}\right)$together with the obvious canonical isomorphism $K(M[1])=(K M)[1]$ is an equivalence of triangulated categories.

(ii) We have $K(M\langle n\rangle)=(K M)[-n]\langle-n\rangle$, canonically.

(iii) For any $p \in k$ we have $K\left(A_{0} p\right)=A^{!} p$ and $K\left(A^{\circledast} p\right)=A_{0}^{!} p$.

Proof. (i) has been done already. (ii) is left to the reader. Just note that the isomorphism is given in essence by $(-1)^{n q}: A^{!} \otimes M^{q} \rightarrow A^{!} \otimes M^{q}$. In (iii) the first equality is evident, and the second follows from the fact that the Koszul complex is a resolution of $k=A_{0}$.

Remark. As can be seen from (ii) already, the standard $t$-structures on our two triangulated categories do not correspond under $K$. In section 2.13 we discuss the non-standard $t$-structure on $D^{\uparrow}\left(A^{!}\right)$coming from the standard one on $D^{\downarrow}(A)$ via $K$, and in section 2.14 we will define the notion of a "Koszul module" and see that $A^{!}-\bmod ^{\uparrow} \cap K\left(A-\bmod ^{\downarrow}\right)$ consists exactly of the Koszul modules for $A^{!}$.

Recall that for a graded ring $B$ we let $B$-mof be the category of finitely generated graded modules. We want to give a version of Koszul duality involving only standard derived categories.

Theorem 2.12.6. Let $A$ be a Koszul ring over $k$. Suppose $A$ is a finitely generated $k$-module both from the left and from the right, so that $A_{i}=0$ for $i \gg 0$. Suppose in addition that $A^{!}$is left noetherian. Then Koszul duality induces an equivalence of triangulated categories

$$
K: D^{b}(A \text {-mof }) \rightarrow D^{b}\left(A^{!} \text {-mof }\right) .
$$

Proof [Theorem]. We must first make precise the meaning of "induces". For this we will characterize $D^{b}(A$-mof $)$ and $D^{b}\left(A^{!}\right.$-mof $)$as certain full subcategories of $D^{\downarrow}(A)$ and $D^{\uparrow}\left(A^{!}\right)$respectively.

Namely, define full subcategories $D_{e}^{\downarrow}(A) \subset D^{\downarrow}(A)$ (resp. $\left.C_{e}^{\downarrow}(A) \subset C^{\downarrow}(A)\right)$ to consist of all objects with only finitely many nonvanishing cohomology groups which are in addition finitely generated $A$-modules. We may also interpret $D_{e}^{\downarrow}(A)$ as the localization of $C_{e}^{\downarrow}(A)$ at quasi-isomorphisms.

Lemma 2.12.7. Suppose $A$ is finitely generated as a left $k$-module, so in particular is left noetherian. Then the obvious functor $D^{b}(A$-mof $) \rightarrow D_{e}^{\downarrow}(A)$ is an equivalence.

Proof. First note that by [Har66, exercise to Prop. 4.8 of Chapter I] the canonical functor $D^{-}(A$-mof $) \rightarrow D_{A \text {-mof }}^{-}(A$-mod $)$ is an equivalence. Thus there is an equivalence $D^{b}(A$-mof $) \rightarrow D_{A \text {-mof }}^{b}(A$-mod $)$. Now $D_{A \text {-mof }}^{b}(A$-mod $)$ can be interpreted as the localization of a suitable homotopy category of complexes $C_{A \text {-mof }}^{b}(A$-mod $)$ at quasiisomorphisms. Also $D_{e}^{\downarrow}(A)$ is the localization of $C_{e}^{\downarrow}(A)$ at quasi-isomorphisms.

Now define a functor $C_{e}^{\downarrow}(A) \rightarrow D_{A \text {-mof }}^{b}(A$-mod $)$ by $X \mapsto \lim \tau \leq i{ }^{\leq} X$ with $\tau \leq i$ the usual truncation functors. The inductive system stabilizes, so there is no problem. This gives a functor $D_{e}^{\downarrow}(A) \rightarrow D_{A \text {-mof }}^{b}(A$-mod $)$. 
In the other direction we have the functor $C_{A \text {-mof }}^{b}(A$-mod $) \rightarrow D_{e}^{\downarrow}(A)$ given by $X \mapsto \lim X / G_{j} X$, using the notation $G_{j}$ from section 2.4 . The projective system stabilizes, and we get a functor $D_{A \text {-mof }}^{b}(A$-mod $) \rightarrow D_{e}^{\downarrow}(A)$.

One may check that these are inverse of one another and that the canonical functor from the lemma is the composition of equivalences $D^{b}(A$-mof $) \rightarrow$ $D_{A \text {-mof }}^{b}(A$-mod $) \rightarrow D_{e}^{\downarrow}(A)$.

Define further the full subcategories $D_{e}^{\uparrow}\left(A^{!}\right) \subset D^{\uparrow}\left(A^{!}\right)\left(\right.$resp. $\left.C_{e}^{\uparrow}\left(A^{!}\right) \subset C^{\uparrow}\left(A^{!}\right)\right)$ to consist of all complexes with only finitely many nonvanishing cohomology groups which are in addition finitely generated $A^{!}$-modules. We may also interpret $D_{e}^{\uparrow}\left(A^{!}\right)$ as the localization of $C_{e}^{\uparrow}\left(A^{!}\right)$at quasi-isomorphisms.

Lemma 2.12.8. Suppose $A^{!}$is left noetherian. Then the obvious functor from $D^{b}\left(A^{!}\right.$-mof $)$to $D_{e}^{\uparrow}\left(A^{!}\right)$is an equivalence.

Proof. First of all we have as before $D^{b}\left(A^{!}-\right.$mof $)=D_{A^{!} \text {-mof }}^{b}\left(A^{!}\right.$-mod $)$. Now we may define a functor $C_{e}^{\uparrow}\left(A^{!}\right) \rightarrow D_{A !-\operatorname{mof}}^{b}\left(A^{!}\right.$-mod) by $X \mapsto \lim \tau^{\geq i} X$ with $\tau^{\geq i}$ the usual truncation functors. The projective system stabilizes and we get a functor $D_{e}^{\uparrow}\left(A^{!}\right) \rightarrow D_{A^{!} \text {-mof }}^{b}\left(A^{!}\right.$-mod $)$.

In the other direction we have the functor $C_{A^{!} \text {-mof }}^{b}\left(A^{!}\right.$-mod $) \rightarrow D_{e}^{\uparrow}\left(A^{!}\right)$given by $X \mapsto \lim G_{i} X$. The injective system stabilizes and gives a functor $D_{A^{!} \text {-mof }}^{b}\left(A^{!}\right.$-mod $)$ $\rightarrow D_{e}^{\uparrow}\left(A^{!}\right)$.

One may check that these are inverse of one another and that the canonical functor from the lemma is the composition of equivalences $D^{b}\left(A^{!}\right.$-mof $) \rightarrow$ $D_{A^{!}-\text {mof }}^{b}\left(A^{!}\right.$-mod $) \rightarrow D_{e}^{\uparrow}\left(A^{!}\right)$.

Now we actually prove the theorem. The equivalence $D^{b}(A$-mof $) \rightarrow D_{e}^{\downarrow}(A)$ makes it clear that $K: D^{\downarrow}(A) \rightarrow D^{\uparrow}\left(A^{!}\right)$maps $D_{e}^{\downarrow}(A)$ to $D_{e}^{\uparrow}\left(A^{!}\right)$and thus induces a fully faithful functor $K: D^{b}(A$-mof $) \rightarrow D^{b}\left(A^{!}\right.$-mof $)$. The only problem is to show that here $K$ is essentially surjective. Since we know that $K A_{0}=A^{!}$, this will follow once we show that $A^{!}$-mof has finite homological dimension. So let us prove this.

Since $K A^{*}=A_{0}^{!}=k$, we have $\operatorname{ext}_{A^{!}}^{i}(k, k\langle j\rangle)=\operatorname{hom}_{D^{b}}\left(A^{*}, A^{*}[i-j]\langle-j\rangle\right)$. Since $A^{*} \in A$-mof is the injective hull of $A_{0}=k$, we actually find that $\operatorname{ext}_{A^{!}}^{i}(k, k\langle j\rangle)$ vanishes for $i \neq j$, and $\operatorname{ext}_{A^{!}}^{i}(k, k\langle i\rangle)=\operatorname{hom}_{A}\left(A^{*}, A^{*}\langle-i\rangle\right)=\operatorname{hom}_{k}\left(A^{*}\langle i\rangle, k\right)=$ $\left(A_{i}^{*}\right)^{*}$. In particular, there exists $d$ such that for all $M, N \in A^{!}$-mod which are finitely generated over $k$ we have $\operatorname{ext}_{A^{!}}^{i}(M, N)=0$ if $i \geq d$.

Now let $M \in A^{!}$-mof be arbitrary. By Lemma 2.1.2, $\operatorname{ext}_{A^{!}}^{i}\left(G_{1} M, k\right)=0 \forall i$. But $M / G_{1} M$ is finitely generated over $k$, so $\operatorname{ext}_{A^{!}}^{i}(M, k)=0$ if $i \geq d$.

Now choose a minimal projective resolution

$$
\ldots \rightarrow P_{2} \rightarrow P_{1} \rightarrow P_{0} \rightarrow M
$$

of $M$ in $A^{!}$-mof. This means precisely that $\operatorname{hom}_{A^{!}}\left(P_{i}, k\langle j\rangle\right)=\operatorname{ext}_{A^{!}}^{i}(M, k\langle j\rangle)$. We deduce that $P_{i}=0$ for all $i \geq d$. The theorem is proven.

2.13. t-structures. Let $A$ be a left finite Koszul ring and consider the Koszul duality functor

$$
K: D^{\downarrow}(A) \rightarrow D^{\uparrow}\left(A^{!}\right)
$$

defined in section 2.12. Here both sides have standard t-structures with cores $A$-mod ${ }^{\downarrow}$ and $A^{!}$-mod $^{\uparrow}$ respectively, but under $K$ these do not correspond. So transporting our standard t-structures via $K$, we obtain non-standard t-structures on 
both $D^{\downarrow}(A)$ and $D^{\uparrow}\left(A^{!}\right)$. We will describe this new t-structure on $D^{\uparrow}\left(A^{!}\right)$quite explicitly. The case of $D^{\downarrow}(A)$ is very similar and will be left to the reader.

First let $B$ be any positively graded ring such that $B_{0}$ is semisimple. In $D^{\uparrow}(B)$ consider the subcategories $D^{\uparrow}(B)^{\leq 0, g}$, resp. $D^{\uparrow}(B)^{\geq 0, g}$, of all objects isomorphic to complexes of graded projective modules

$$
\ldots \rightarrow P^{i} \rightarrow P^{i+1} \rightarrow \ldots
$$

in $C^{\uparrow}(B)$ such that $P^{i}$ is generated by its components of degree $\leq-i$, resp. $\geq-i$, for all $i$.

Proposition 2.13.1. The pair $\left(D^{\uparrow}(B)^{\leq 0, g}, D^{\uparrow}(B)^{\geq 0, g}\right)$ is a $t$-structure on $D^{\uparrow}(B)$.

Remark. An analogous definition is used to obtain a non-standard t-structure on $D^{-}(B$-mod). This is the "geometric" t-structure in [Soe92], hence the notation.

Proof [Proposition]. Most of this is straightforward and left to the reader. Let us just prove that every $Y \in D^{\uparrow}(B)$ decomposes into a distinguished triangle $\left(Y^{<0}, Y, Y^{\geq 0}\right)$. Using Lemma 2.1.2 or more precisely its proof, we see that every object of $D^{\uparrow}(B)$ can be represented by a complex of projective objects in $C^{\uparrow}(B)$, say

Now we need

$$
\ldots \rightarrow P^{i} \rightarrow P^{i+1} \rightarrow \ldots
$$

Lemma 2.13.2. This complex can be choosen such that the induced maps

$$
P^{i} / B_{>0} P^{i} \rightarrow P^{i+1} / B_{>0} P^{i+1}
$$

all vanish.

Proof. Let us simplify notation and put $\bar{M}=M / B_{>0} M, k=B_{0}, \otimes=\otimes_{k}$. Remark that for any projective $P \in B$-mod every splitting in $k$-mod of the surjection $P \rightarrow \bar{P}$ defines an isomorphism $P \cong B \otimes \bar{P}$. Consider now the maps $\bar{\partial}: \overline{P^{i}} \rightarrow \overline{P^{i+1}}$. Choose decompositions

$$
\overline{P^{i}}=I^{i} \oplus H^{i} \oplus R^{i}
$$

in $k$-mod such that $I^{i}=\bar{\partial}\left(\overline{P^{i}}\right)$ and $I^{i} \oplus H^{i}=\operatorname{ker}(\bar{\partial})$, so that $\bar{\partial}$ induces isomorphisms $R^{i} \rightarrow I^{i+1}$. Now choose splittings of graded $k$-modules $R^{i} \rightarrow P^{i}$. This leads to splittings $I^{i+1} \rightarrow P^{i+1}$, and we can extend these splittings from $I^{i} \oplus R^{i}$ to all of $\overline{P^{i}}$. Thus we can decompose

$$
P^{i}=\left(B \otimes I^{i}\right) \oplus\left(B \otimes H^{i}\right) \oplus\left(B \otimes R^{i}\right)
$$

in such a way that our differentials induce isomorphisms from $\left(B \otimes R^{i}\right)$ to $\left(B \otimes I^{i+1}\right)$. It is then clear that our complex $P^{\bullet}$ is quasi-isomorphic to the complex $\left(B \otimes H^{\bullet}\right)$ with the induced differentials, and that this complex satisfies the conditions of the lemma.

So now let $Y \in D^{\uparrow}(B)$ be represented by a complex of projective objects

$$
\ldots \rightarrow P^{i} \rightarrow P^{i+1} \rightarrow \ldots
$$

in $C^{\uparrow}(B)$ such that if we apply $k \otimes_{B}$ all differentials become zero. Consider the submodules $B P_{<-i}^{i} \subset P^{i}$ generated by the homogeneous components of degree less than $-i$. They are projective, and the quotients $P^{i} / B P_{<-i}^{i}$ are projective as well. Furthermore, the differential has to map $B P_{<-i}^{i}$ to $B P_{<-i-1}^{i+1}$, by our assumption on the complex. Now we just let $Y^{<0}$ be the complex of the $B P_{<-i}^{i}$ and $Y^{\geq 0}$ the complex of the $P^{i} / B P_{<-i}^{i}$, and we are done. The proposition is proved. 
As a corollary of this proof, we give a more direct description of the core of our non-standard t-structure.

Corollary 2.13.3. Any object of the core of our non-standard $t$-structure on $D^{\uparrow}(B)$ is quasi-isomorphic to a complex of projectives

$$
\ldots \rightarrow P^{i} \rightarrow P^{i+1} \rightarrow \ldots
$$

in $C^{\uparrow}(B)$ such that $P^{i}=B P_{-i}^{i} \forall i$.

Now we prove that this is the t-structure we were looking for.

Proposition 2.13.4. The Koszul duality functor $K$ transforms the standard $t$ structure on $D^{\downarrow}(A)$ into our non-standard t-structure $\left(D^{\uparrow}\left(A^{!}\right)^{\leq 0, g}, D^{\uparrow}\left(A^{!}\right)^{\geq 0, g}\right)$ on $D^{\uparrow}\left(A^{!}\right)$.

Proof. Certainly

$$
D^{\downarrow}(A)^{\leq 0}=\left\{X \in D^{\downarrow}(A) \mid \operatorname{Hom}\left(X, A^{\circledast}[i]\langle n\rangle\right)=0 \forall i<0, \forall n\right\}
$$

and $K$ transforms this into

$$
\left\{Y \in D^{\uparrow}\left(A^{!}\right) \mid \operatorname{Hom}\left(Y, A^{\circledast}[i-n]\langle-n\rangle\right)=0 \forall i<0, \forall n\right\} .
$$

It is clear that all $Y \in D^{\uparrow}\left(A^{!}\right) \leq 0, g$ satisfy this condition. It is equally clear that if $Y \in D^{\uparrow}\left(A^{!}\right)^{\geq 0, g}$ satisfies this condition, then any complex of projectives isomorphic to it in the derived category has to be exact, i.e. $Y=0$. Decomposing an arbitrary $Y \in D^{\uparrow}\left(A^{!}\right)$into a distinguished triangle $\left(Y^{<0}, Y, Y^{\geq 0}\right)$ according to our t-structure, we find then that indeed $K\left(D^{\downarrow}(A) \leq 0\right) \subset D^{\uparrow}\left(A^{!}\right) \leq 0, g$.

Similar arguments show the other inclusion $K\left(D^{\downarrow}(A)^{\geq 0}\right) \subset D^{\uparrow}\left(A^{!}\right) \geq 0, g$.

2.14. Koszul modules. Let $A$ be a positively graded ring over the semisimple ring $k=A_{0}$.

Definition 2.14.1. A graded $A$-module $M \in A$-mod is called "Koszul" if and only if $M$ admits a graded projective resolution

$$
\ldots \rightarrow P^{2} \rightarrow P^{1} \rightarrow P^{0} \rightarrow M
$$

such that $P^{i}$ is generated by its component of degree $i, P^{i}=A P_{i}^{i}$.

Proposition 2.14.2. Let $M \in A$-mod be bounded from below, i.e. $M \in A$-mod ${ }^{\uparrow}$. Then the following are equivalent:

(1) $M$ is Koszul.

(2) $\operatorname{ext}_{A}^{i}(M, N)=0$ for any pure module $N$ whose weight is not $(-i)$.

(3) $\operatorname{ext}_{A}^{i}(M, k\langle n\rangle)=0$ unless $i=n$.

Proof. Almost identical to the proof of Proposition 2.1.3, and so we leave it to the reader.

Definition 2.14.3. A graded $A$-module $M \in A$-mod is called "quadratic" if and only if it is generated in degree zero with relations in degree one.

We may spell this out as follows: The multiplication $m: A \otimes M_{0} \rightarrow M$ is a surjection, and $\operatorname{ker}(m)$ is generated as a left $A$-module by its component of degree one $S=\operatorname{ker}\left(A_{1} \otimes M_{0} \rightarrow M\right)$, so that $M=A \otimes M_{0} / A S$.

Proposition 2.14.4. Any Koszul module is quadratic.

Proof. This is trivial. 
Suppose from now on that $A$ is a quadratic ring, $A=T_{k} V /(R)$. Extending the considerations of section 2.6, we may define the Koszul complex

$$
\ldots \rightarrow K^{2} M \rightarrow K^{1} M \rightarrow K^{0} M
$$

of a quadratic $A$-module $M$ by setting

$$
K^{i} M=A \otimes\left(\bigcap_{\nu} V^{\otimes \nu} \otimes R \otimes V^{\otimes i-\nu-2} \otimes M_{0} \cap V^{\otimes i-1} \otimes S\right) .
$$

Proposition 2.14.5. A quadratic module $M$ over a quadratic ring $A$ is Koszul if and only if its Koszul complex $K^{\bullet} M$ is a resolution of $M$.

Proof. Almost the same as the proof of Theorem 2.6.1, and so we leave it to the reader.

If $A$ is a left finite quadratic ring and $M$ a finitely generated quadratic module over $A$, we may form the quadratic dual $M^{!} \in \bmod -A^{!}$given by $S^{\perp} \subset\left(A_{1} \otimes M_{0}\right)^{*}=$ $M_{0}^{*} \otimes A_{1}^{*}$ as $M^{!}=M_{0}^{*} \otimes A^{!} / S^{\perp} A^{!}$. Just as in section 2.8 we may then identify the Koszul complex of $M$ with the complex

$$
\ldots \rightarrow A \otimes{ }^{*}\left(M_{2}^{!}\right) \rightarrow A \otimes{ }^{*}\left(M_{1}^{!}\right) \rightarrow A \otimes{ }^{*}\left(M_{0}^{!}\right)
$$

where we leave the definition of the differential to the reader. In case $A$ is Koszul, Theorem 2.10.1 generalizes to a canonical isomorphism $M^{!}=\operatorname{Ext}_{A}^{\bullet}(M, k)$ of left modules over $\left(A^{!}\right)^{\text {opp }}=\operatorname{Ext}_{A}^{\bullet}(k, k)$.

There also is an analog of the distributivity criterion (explained in the remark closing subsection 2.6) for Koszul modules over Koszul rings. It shows in particular that the dual $M^{!}$of a finitely generated Koszul module over a left finite Koszul ring is also Koszul.

\section{Parabolic-Singular duality and Koszul Duality}

3.1. Notation from algebraic geometry. Let $X$ be a complex algebraic variety. Then we denote by $\mathcal{D}(X)=D_{\text {const }}^{b}\left(S h\left(X^{a n}\right)\right)$ the bounded derived category with algebraically constructible cohomology of the category $S h\left(X^{a n}\right)$ of sheaves of complex vector spaces on the analytic space $X^{a n}$ defined by $X$. Let $\mathcal{P}(X) \subset \mathcal{D}(X)$ be the perverse sheaves (for the middle perversity) and $H^{i}: \mathcal{D}(X) \rightarrow \mathcal{P}(X)$ the perverse cohomology functors. For any $\mathcal{F}, \mathcal{G} \in \mathcal{D}(X)$ we may form the derived

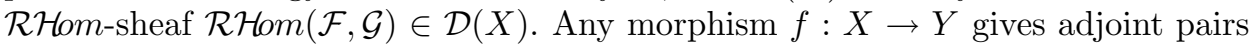
of exact functors $\left(f^{*}, f_{*}\right)$ and $\left(f_{!}, f^{!}\right)$relating $\mathcal{D}(X)$ and $\mathcal{D}(Y)$. More classically $f^{*}$,

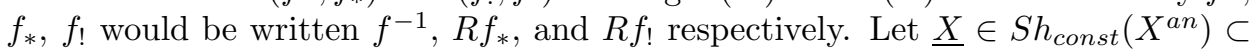
$\mathcal{D}(X)$ be the constant sheaf $\mathbb{C}$. Let Hom Don $_{\mathcal{D}}$ denote homomorphisms in $\mathcal{D}(X)$, and put $\operatorname{Hom}_{\mathcal{D}}^{i}(\mathcal{F}, \mathcal{G})=\operatorname{Hom}_{\mathcal{D}}(\mathcal{F}, \mathcal{G}[i])$ for all $\mathcal{F}, \mathcal{G} \in \mathcal{D}(X)$. For any $\mathcal{F} \in \mathcal{D}(X)$ its hypercohomology $\mathbb{H}^{\bullet} \mathcal{F}=\bigoplus \mathbb{H}^{i} \mathcal{F}$ is a graded $\mathbb{C}$-vectorspace and even a graded module over the cohomology ring $H^{\bullet}(X)$ of $X$ (which should really be denoted $\mathbb{H}^{\bullet}(\underline{X})$ or even $\left.\operatorname{End}_{\mathcal{D}}^{\bullet}(\underline{X}, \underline{X})\right)$. More details on these notions may be found in [Bor84, KS90, BBD82].

3.2. Existence of projective objects in some abelian categories. To prove that suitable categories of perverse sheaves have enough projectives, we first work in an abstract context. The results in this and the next section can also be found in a paper by Cline, Parshall, and Scott [CPS91]. Let $k$ be a field and $\mathcal{A}$ an abelian $k$-category. Suppose that

(1) Every object of $\mathcal{A}$ has finite length. 
(2) There are only finitely many simple isomorphism classes in $\mathcal{A}$. To simplify the exposition, suppose furthermore

(3) The endomorphisms of simple objects in $\mathcal{A}$ are reduced to scalars. Let $\{L(s)\}_{s \in S}$ represent the simple isomorphism classes in $\mathcal{A}$. Assume a partial order $\leq$ is given on $S$ and $S$ is equipped with the order topology, i.e. closed subsets $T \subset S$ are characterized by $s \in T, s^{\prime} \leq s \Rightarrow s^{\prime} \in T$. For any closed $T \subset S$ let $\mathcal{A}_{T}$ be the full subcategory of $\mathcal{A}$ of objects "supported on $T$ ", i.e. all of whose simple subquotients have parameter in $T$.

Assume we are given for all $s \in S$ objects $\Delta(s), \nabla(s)$ and morphisms $\Delta(s) \rightarrow L(s), L(s) \rightarrow \nabla(s)$ in $\mathcal{A}$ such that

(4) Whenever $T \subset S$ is closed and $s \in T$ is maximal, $\Delta(s) \rightarrow L(s)$ is a projective cover and $L(s) \rightarrow \nabla(s)$ an injective hull of $L(s)$ in $\mathcal{A}_{T}$. (In particular, both $\Delta(s)$ and $\nabla(s)$ are indecomposable. $)$

(5) $\operatorname{ker}(\Delta(s) \rightarrow L(s))$ and $\operatorname{coker}\left(L(s) \rightarrow \nabla(s)\right.$ lie in $\mathcal{A}_{<s} \forall s \in S$. Assume furthermore

(6) $\operatorname{Ext}_{\mathcal{A}}^{2}(\Delta(s), \nabla(t))=0 \quad \forall s, t \in S$.

We call the $\Delta(s)$ the standard modules and $\nabla(s)$ the costandard modules.

Theorem 3.2.1. Let $\mathcal{A}$ be an abelian category satisfying all of the above conditions (1)-(6). Then $\mathcal{A}$ has enough projective objects, and each projective has a finite filtration with standard subquotients.

Remarks. (1) If we let $P(s) \rightarrow L(s)$ be the projective cover and $(P(s): \Delta(t))$ the multiplicity of $\Delta(t))$ as a subquotient in a standard filtration of $P(s)$, then the so-called reciprocity formula $(P(s): \Delta(t))=[\nabla(t): L(s)]$ holds. Indeed, it is easy to see that both sides are just $\operatorname{dim}_{k} \operatorname{Hom}_{\mathcal{A}}(P(s), \nabla(t))$.

(2) Dual results hold for injectives.

(3) As Ringel explained to one of the authors, the conditions (1)-(5) are already sufficient to ensure the existence of enough projectives. His argument goes as follows. Choose $s \in S$ maximal and assume by induction that the statement is known for $\mathcal{A}_{S-\{s\}}$. For any $t \in S$ we need to show that there is a bound $N(t)$ on the possible length of an object $X$ with unique simple quotient $L(t)$. For $t=s$ this is clear, so suppose $t \neq s$. Consider the short exact sequence

$$
0 \rightarrow K \rightarrow X \rightarrow \bar{X} \rightarrow 0,
$$

where $\bar{X}$ is the maximal quotient of $X$ which lies in $\mathcal{A}_{S-\{s\}}$. Then $\bar{X}$ is a quotient of the projective cover of $L(t)$ in $\mathcal{A}_{S-\{s\}}$, so its length is bounded. Furthermore, we have an inclusion

$$
\operatorname{Hom}(K, L(s)) \hookrightarrow \operatorname{Ext}^{1}(\bar{X}, L(s))
$$

and $K / \operatorname{rad} K$ is a direct sum of copies of $L(s)$, hence $K$ is a quotient of a direct sum of at most $\operatorname{dim} \operatorname{Ext}^{1}(\bar{X}, L(s))$ copies of $\Delta(s)$. This does the job.

Proof. We start the proof by deriving further properties of $\mathcal{A}$. Let us henceforth abreviate $\operatorname{Hom}_{\mathcal{A}}, \operatorname{Ext}_{\mathcal{A}}^{i}$, End $\operatorname{An}_{\mathcal{A}}$ by Hom, $\mathrm{Ext}^{i}$, End.

(7) $\operatorname{End}(\Delta(s))=k=\operatorname{End}(\nabla(s))$ follows from (3), (4) and (5).

(8) For all $M, N \in \mathcal{A}$ the space $\operatorname{Hom}(M, N)$ is of finite dimension, as follows from (3) and (1).

(9) For all $M, N \in \mathcal{A}$ the space $\operatorname{Ext}^{1}(M, N)$ is of finite dimension. To prove this, we may assume without restriction that $M, N$ are simple. First assume 
$M=L(s), N=L(t)$ with $t \ngtr s$. Then there exists a closed subset $T \subset S$ such that $s, t \in T$ and $s$ is maximal in $T$. Now the short exact sequence ker $\hookrightarrow$ $\Delta(s) \rightarrow L(s)$ in $\mathcal{A}_{T}$ gives us a surjection Hom $(\operatorname{ker}, L(t)) \rightarrow \operatorname{Ext}^{1}(L(s), L(t))$, and we are done by (8). If $t>s$ we proceed dually.

To prove Theorem 3.2.1, we prove more generally for any closed subset $T \subset S$ :

Claim $(T)$. There are enough projectives in $\mathcal{A}_{T}$, and each one of those has a finite filtration with standard sucessive quotients.

For this we proceed by induction on $|T|$. So let $T \subset S$ be closed and $s \in S$ such that $T^{\prime}=T \cup\{s\}$ is closed as well. We assume claim $(T)$ and try to construct for all $t \in T^{\prime}$ a projective cover $P_{T^{\prime}}(t) \rightarrow L(t)$ of $L(t)$ in $\mathcal{A}_{T^{\prime}}$. If $t=s$, then $\Delta(s) \rightarrow L(s)$ is the sought-for cover by (4). If $t \neq s$, then $t \in T$ and claim $(T)$ gives at least a projective cover $P_{T}(t) \rightarrow L(t)$ of $L(t)$ in $\mathcal{A}_{T}$.

Let us abbreviate $P_{T}(t)=P$. Put $E=\operatorname{Ext}^{1}(P, \Delta(s))$. Remark that for any finite dimensional $k$-vectorspace $F$ and any object $M$ of a $k$-category we may formally define the object $F \otimes M$. The canonical element in $E^{*} \otimes E=E^{*} \otimes \operatorname{Ext}^{1}(P, \Delta(s))=$ $\operatorname{Ext}^{1}\left(P, E^{*} \otimes \Delta(s)\right)$ gives rise to an extension $E^{*} \otimes \Delta(s) \hookrightarrow \tilde{P} \rightarrow P$ such that, if we take the long exact sequence $\ldots \rightarrow \operatorname{Hom}\left(E^{*} \otimes \Delta(s), \Delta(s)\right) \rightarrow \operatorname{Ext}^{1}(P, \Delta(s)) \rightarrow$ $\operatorname{Ext}^{1}(\tilde{P}, \Delta(s)) \rightarrow \operatorname{Ext}^{1}\left(E^{*} \otimes \Delta(s), \Delta(s)\right) \rightarrow \ldots$, then the diagram

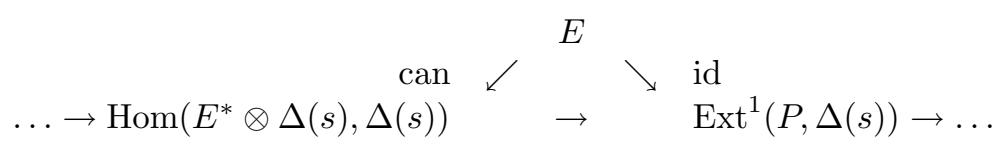

commutes. Since can is an isomorphism by (7), the above boundary map is actually an isomorphism. Since $\operatorname{Ext}^{1}(\Delta(s), \Delta(s))=0$ by $(4)$, we get $\operatorname{Ext}^{1}(\tilde{P}, \Delta(s))=0$. We want to prove that $\tilde{P}$ is a projective cover of $L(t)$ in $\mathcal{A}_{T^{\prime}}$. This would establish claim $\left(T^{\prime}\right)$. To achieve this we only have to prove that, for $r \in T^{\prime}=T \cup\{s\}$,

$$
\begin{aligned}
\operatorname{dim} \operatorname{Hom}(\tilde{P}, L(r)) & = \begin{cases}1, & r=t \\
0, & \text { else }\end{cases} \\
\operatorname{Ext}^{1}(\tilde{P}, L(r)) & =0
\end{aligned}
$$

Suppose first that $r \in T$. We consider the short exact sequence $E^{*} \otimes \Delta(s) \hookrightarrow \tilde{P} \rightarrow P$. Applying $\operatorname{Ext}^{i}(, L(r))$, we get a long exact sequence. Since

$$
\operatorname{Hom}\left(E^{*} \otimes \Delta(s), L(r)\right)=\operatorname{Ext}^{1}\left(E^{*} \otimes \Delta(s), L(r)\right)=0
$$

by (4), this settles the case $r \in T$.

So we are left with the task of proving that

$$
\operatorname{Hom}(\tilde{P}, L(s))=\operatorname{Ext}^{1}(\tilde{P}, L(s))=0 .
$$

Consider the short exact sequence ker $\hookrightarrow \Delta(s) \rightarrow L(s)$. It gives rise to two long exact sequences

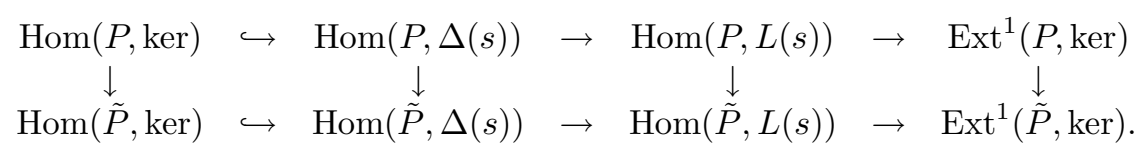

By the case $r \in T$ the first vertical is an isomorphism and both Ext-groups vanish. By construction of $\tilde{P}$ the second vertical is an isomorphism. Thus the third vertical is an isomorphism and $\operatorname{Hom}(\tilde{P}, L(s))=\operatorname{Hom}(P, L(s))=0$. 
Next we prove $\operatorname{Ext}^{1}(\tilde{P}, L(s))=0$. The short exact sequence ker $\hookrightarrow \Delta(s) \rightarrow$ $L(s)$ gives an exact sequence $\operatorname{Ext}^{1}(\tilde{P}, \Delta(s)) \rightarrow \operatorname{Ext}^{1}(\tilde{P}, L(s)) \rightarrow \operatorname{Ext}^{2}(\tilde{P}$, ker $)$. We deduced already from the definition of $\tilde{P}$ that $\operatorname{Ext}^{1}(\tilde{P}, \Delta(s))=0$. To get $\operatorname{Ext}^{2}(\tilde{P}$, ker $)=0$ it is sufficient to prove that $\operatorname{Ext}^{2}(\tilde{P}, L(r))=0$ for all $r \in T$. But this follows via the short exact sequence $L(r) \hookrightarrow \nabla(r) \rightarrow$ coker since we know already that $\operatorname{Ext}^{1}(\tilde{P}$, coker $)=0$, and since $\operatorname{Ext}^{2}(\tilde{P}, \nabla(r))=0$ by $(6)$ because $\tilde{P}$ has a filtration with standard subquotients. So $\operatorname{Ext}^{2}(\tilde{P}$, ker $)=0$, and thus $\operatorname{Ext}^{1}(\tilde{P}, L(s))=0$.

Corollary 3.2.2. Let $\mathcal{A}$ be an abelian category satisfying (1)-(6). Then $\mathcal{A}$ has finite cohomological dimension.

Proof. We have to prove that every object admits a projective resolution of finite length. It is clear (at least from the point of view of derived categories) that if this holds for two objects in a short exact sequence, then it also holds for the third one. Hence it is enough to prove this for simple objects, and using (5) and induction over $S$ from below we see that it is also enough to prove it for standard objects. But then we may use induction over $S$ from above, because the projectives $P(s)$ we just constructed have filtrations with a single subquotient $\Delta(s)$ and all the other subquotients of the form $\Delta(t), t>s$.

To check for condition (6), sometimes the following lemmas from folklore are helpful.

Lemma 3.2.3. Let $\mathcal{A}$ be an abelian category and $\mathcal{B} \subset \mathcal{A}$ a full abelian subcategory, closed under extensions and such that the inclusion is an exact functor. Then for all $M, N \in \mathcal{B}$ the canonical map

$$
\operatorname{Ext}_{\mathcal{B}}^{2}(M, N) \rightarrow \operatorname{Ext}_{\mathcal{A}}^{2}(M, N)
$$

is an injection.

Proof. Suppose given $e \in \operatorname{Ext}_{\mathcal{B}}^{2}(M, N), e \neq 0$. Certainly there exists a surjection $P \rightarrow M$ in $\mathcal{B}$ such that the pull-back $\operatorname{Ext}_{\mathcal{B}}^{2}(M, N) \rightarrow \operatorname{Ext}_{\mathcal{B}}^{2}(P, N)$ maps $e$ to zero. The short exact sequence ker $\hookrightarrow P \rightarrow M$ gives a commutative diagram with exact rows

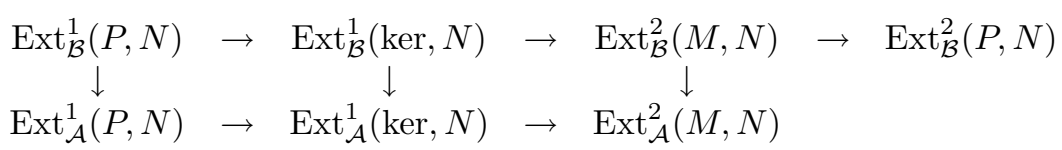

and a diagram chase proves the lemma.

Now consider any triangulated category $\mathcal{D}$ with t-structure and let $\mathcal{P} \subset \mathcal{D}$ be the core. In general one cannot extend the embedding $\mathcal{P} \subset \mathcal{D}$ to a triangulated functor $D^{b}(\mathcal{P}) \subset \mathcal{D}$ in a canonical way. However we have for all $M, N \in \mathcal{P}$ canonical maps $\operatorname{Ext}_{\mathcal{P}}^{i}(M, N) \rightarrow \operatorname{Hom}_{\mathcal{D}}^{i}(M, N)$. This follows from the fact that for fixed $M$ both sides are $\delta$-functors in $N$ from $\mathcal{P}$ to the category of abelian groups, and the left hand side is even a universal $\delta$-functor.

Lemma 3.2.4. In the situation above, the canonical map

$$
\operatorname{Ext}_{\mathcal{P}}^{2}(M, N) \rightarrow \operatorname{Hom}_{\mathcal{D}}^{2}(M, N)
$$

is an injection.

Proof. Very similar to the proof of the previous lemma. 
3.3. Existence of projective perverse sheaves. We want to prove that suitable categories of perverse sheaves have enough projective objects. This is due to [MV87]. However we think that our argument is easier. In fact, it is quite similar to an argument of [CPS91].

Let $X$ be a complex algebraic variety with an algebraic stratification by affine linear spaces $X=\dot{\bigcup}_{w \in \mathcal{W}} X_{w}$. Let $\mathcal{D}(X, \mathcal{W}) \subset \mathcal{D}(X)$ consist of all objects which are smooth along the stratification and set $\mathcal{P}(X, \mathcal{W})=\mathcal{P}(X) \cap \mathcal{D}(X, \mathcal{W})$. Let $j_{w}$ : $X_{w} \rightarrow X$ be the inclusions of the strata. Put $|w|=\operatorname{dim} X_{w}$, so $X_{w} \cong \mathbb{C}^{|w|}$. Let $\mathcal{C}_{w}=\underline{X}_{w}[-|w|]$ be the constant perverse sheaf on $X_{w}$, and consider the standard and costandard objects $\mathcal{M}_{w}=j_{w !} \mathcal{C}_{w}, \mathcal{N}_{w}=j_{w *} \mathcal{C}_{w}$. By our assumptions $j_{w}$ is affine, hence both $\mathcal{M}_{w}$ and $\mathcal{N}_{w}$ belong to $\mathcal{P}(X, \mathcal{W})$. We will need

Theorem 3.3.1. There are enough projectives in $\mathcal{P}(X, \mathcal{W})$, and each one has a filtration with standard objects as subquotients. Dually, there also are enough injectives in $\mathcal{P}(X, \mathcal{W})$, and each one has a filtration with costandard objects as subquotients.

Proof. This is a direct consequence of Theorem 3.2.1.

For simplicity suppose now, in addition, that $X$ is smooth. Then we may interpret $\mathcal{P}(X)$ as a category of $\mathcal{D}$-modules and $\mathcal{D}(X)$ as a category of complexes of $\mathcal{D}$ modules, and in this way get an evident extension of the embedding $\mathcal{P}(X) \rightarrow \mathcal{D}(X)$ to a triangulated functor $D^{b}(\mathcal{P}(X)) \rightarrow \mathcal{D}(X)$. This in turn gives us in an obvious way a functor $D^{b}(\mathcal{P}(X, \mathcal{W})) \rightarrow \mathcal{D}(X, \mathcal{W})$, and under our assumptions we obtain

Corollary 3.3.2. The obvious functor $D^{b}(\mathcal{P}(X, \mathcal{W})) \rightarrow \mathcal{D}(X, \mathcal{W})$ is an equivalence of categories.

Proof. By Corollary 3.2.2 we see that the left hand side is generated as a triangulated category by the projectives and also by the injectives in $\mathcal{P}(X, \mathcal{W})$. So we need only show that for $P, I \in \mathcal{P}(X, \mathcal{W})$ a projective (resp. injective) object we have $\operatorname{Hom}_{D^{b}(\mathcal{P}(X, \mathcal{W}))}^{i}(P, I)=\operatorname{Hom}_{\mathcal{D}}^{i}(P, I)$. This is clear for $i=0$, and for $i \neq 0$ both sides vanish: the left hand side since it is just an Ext group in $\mathcal{P}(X, \mathcal{W})$, the right hand side since $P$ (resp. $I$ ) has a filtration with standard (resp. costandard) sucessive quotients and $\operatorname{Hom}_{\mathcal{D}}^{i}\left(\mathcal{M}_{v}, \mathcal{N}_{w}\right)=0$ for $i \neq 0$.

3.4. Calculation of some extensions. Consider a complex algebraic variety $X$ filtered by closed subvarieties $X=X_{0} \supset X_{1} \supset \ldots \supset X_{r}=\emptyset$ and $\mathcal{F} \in \mathcal{D}(X)$. To compute $\mathbb{H} \bullet \mathcal{F}$ we have to choose an injective resolution $I^{\bullet}$ of $\mathcal{F}$ and take cohomology of the complex of global sections $\Gamma\left(I^{\bullet}\right)$. This is filtered by supports, $0 \subset \Gamma_{X_{r}}\left(I^{\bullet}\right) \subset$ $\ldots \subset \Gamma_{X_{0}}\left(I^{\bullet}\right)=\Gamma\left(I^{\bullet}\right)$. So its cohomology is the limit of a spectral sequence with $E_{1}$-term $E_{1}^{p, q}=\mathbb{H}_{X_{p}-X_{p+1}}^{p+q}(\mathcal{F})$, the local hypercohomology of $\mathcal{F}$ along $X_{p}$ $X_{p+1}$. For $v: Y \hookrightarrow X$ locally closed we have $\mathbb{H}_{Y}^{\bullet}(\mathcal{F})=\mathbb{H} \bullet v^{!} \mathcal{F}$. So if we let $i_{p}$ : $\left(X_{p}-X_{p+1}\right) \hookrightarrow X$ be the inclusion, the $E_{1}$-term can also be written as $E_{1}^{p, q}=$ $\mathbb{H}^{p+q} i_{p}^{!} \mathcal{F}$. For $\mathcal{L}, \mathcal{M} \in \mathcal{D}(X)$ we then find that $\operatorname{Hom} \bullet(\mathcal{L}, \mathcal{M})=\mathbb{H} \bullet \mathcal{R H o m}(\mathcal{L}, \mathcal{M})$ is the limit of a spectral sequence with $E_{1}$-term $E_{1}^{p, q}=\mathbb{H}^{p+q} i_{p}^{!} \mathcal{R} \mathcal{H} \operatorname{Hom}(\mathcal{L}, \mathcal{M})=$ $\mathbb{H}^{p+q} \mathcal{R} \mathcal{H o m}\left(i_{p}^{*} \mathcal{L}, i_{p}^{!} \mathcal{M}\right)$.

Now let $X$ be a complex variety with an algebraic stratification by affine linear spaces $X=\dot{\bigcup}_{w \in \mathcal{W}} X_{w}$. Let $j_{w}: X_{w} \rightarrow X$ be the inclusions of the strata. Put $|w|=\operatorname{dim} X_{w}$, so $X_{w} \cong \mathbb{C}^{|w|}$. Consider the intersection cohomology complexes $\mathcal{I C}_{w} \in \mathcal{P}(X)$ of the $\bar{X}_{w}$. For all $i$ the perverse cohomology $H^{-i} j_{v}^{*} \mathcal{I} \mathcal{C}_{w} \in \mathcal{P}\left(X_{v}\right)$ is 
of the form $H^{-i} j_{v}^{*} \mathcal{I} \mathcal{C}_{w} \cong n_{v, w}^{i} \mathcal{C}_{v}$ where $\mathcal{C}_{v} \in \mathcal{P}\left(X_{v}\right)$ is the constant sheaf put in degree $-|v|$ and $n_{v, w}^{i} \in \mathbb{N}$ are multiplicities. We may assemble the constants $n_{v, w}^{i}$ together in a $\mathcal{W} \times \mathcal{W}$-matrix $I C(t)=I C(X, t)$ with entries in $\mathbb{Z}[t]$ given by

$$
I C_{v, w}(t)=\sum_{i} n_{v, w}^{i} t^{i}
$$

In fact, since the $j_{v}^{*}$ are right exact for the perverse t-structure, $n_{v, w}^{i}=0$ for $i<0$. By definition the diagonal entries of our matrix $I C(t)$ are all one, and for an ordering of $\mathcal{W}$ compatible with the closure relations between strata the whole matrix is upper triangular.

Theorem 3.4.1. Suppose we have parity vanishing, i.e. $H^{i} j_{v}^{*} \mathcal{I} \mathcal{C}_{w}=0$ unless $i \equiv$ $|v|+|w|(\bmod 2)$. Then

$$
\sum_{i} t^{i} \operatorname{dim} \operatorname{Hom}_{\mathcal{D}}^{i}\left(\mathcal{I C}, \mathcal{I C}_{y}\right)=\sum_{z} I C_{z, x}(t) I C_{z, y}(t)
$$

is just the $(x, y)$-entry of $I C^{T} \cdot I C$.

Remark. Instead of parity vanishing we might as well assume pointwise purity of the $\mathcal{I C}_{x}$. The idea to use parity vanishing comes from [CPS91].

Proof. We apply the preceding considerations to $\mathcal{L}=\mathcal{I C}_{x}, \mathcal{M}=\mathcal{I C}_{y}$, and the filtration by the $X_{p}=\bigcup_{|x|+p \leq \operatorname{dim} X} X_{x}$. Thus $\operatorname{Hom}_{\mathcal{D}}\left(\mathcal{I C}_{x}, \mathcal{I C}_{y}\right)$ is the limit of a spectral sequence with $E_{1}$-term

$$
\begin{aligned}
E_{1}^{p, q} & =\mathbb{H}^{p+q} \mathcal{R H o m}\left(i_{p}^{*} \mathcal{I} \mathcal{C}_{x}, i_{p}^{!} \mathcal{I} \mathcal{C}_{y}\right) \\
& \left.=\oplus_{|z|+p=\operatorname{dim} X} \mathbb{H}^{p+q} \mathcal{R} \mathcal{H o m}_{z}^{*} j_{z}^{*} \mathcal{I C}_{x}, j_{z}^{!} \mathcal{I C}_{y}\right)
\end{aligned}
$$

Our assumptions say that

$$
j_{z}^{*} \mathcal{I} \mathcal{C}_{x} \cong \bigoplus_{\mu} n_{z, x}^{\mu} \mathcal{C}_{z}[\mu]
$$

and dually

$$
j_{z}^{!} \mathcal{I} \mathcal{C}_{y} \cong \bigoplus_{\nu} n_{z, y}^{\nu} \mathcal{C}_{z}[-\nu]
$$

and now parity vanishing tells us that our spectral sequence vanishes "like a chessboard". It follows that it degenerates at the $E_{1}$-term, and we deduce that

$$
\operatorname{dim} \operatorname{Hom}_{\mathcal{D}}^{i}\left(\mathcal{I C}_{x}, \mathcal{I C}_{y}\right)=\sum_{p+q=i} \operatorname{dim} E_{1}^{p+q}=\sum_{z, \mu+\nu=i} n_{z, x}^{\mu} n_{z, y}^{\nu}
$$

This proves our theorem.

In the same setup we can also prove

Proposition 3.4.2. Suppose we have parity vanishing. Then hypercohomology induces an injection

$$
\operatorname{Hom}_{\mathcal{D}}^{\bullet}\left(\mathcal{I C}_{x}, \mathcal{I C}_{y}\right) \rightarrow \operatorname{Hom}_{\mathbb{C}}\left(\mathbb{H}^{\bullet} \mathcal{I} \mathcal{C}_{x}, \mathbb{H}^{\bullet} \mathcal{I} \mathcal{C}_{y}\right)
$$

Proof. By parity vanishing the spectral sequence $\mathbb{H}^{p+q} i_{p}^{!} \mathcal{I} \mathcal{C}_{x} \Rightarrow \mathbb{H}^{n} \mathcal{I} \mathcal{C}_{x}$ is degenerate. So if $f \in \operatorname{Hom}_{\mathcal{D}}^{\bullet}\left(\mathcal{I C}_{x}, \mathcal{I C}_{y}\right)$ is given such that $\mathbb{H}^{\bullet} f=0$, then necessarily $0=i_{p}^{!} f \in \operatorname{Hom}_{\mathcal{D}}^{\bullet}\left(i_{p}^{!} \mathcal{I} \mathcal{C}_{x}, i_{p}^{!} \mathcal{I} \mathcal{C}_{y}\right)$ for all $p$. Let $a_{p}: X_{p} \hookrightarrow X$ be the inclusion. We have a decomposition

$$
X_{p}-X_{p+1} \stackrel{u}{\hookrightarrow} X_{p} \stackrel{i}{\hookleftarrow} X_{p+1}
$$


in an open and a closed subset and a distinguished triangle $\left(u_{*} u^{!}\right.$, id,$\left.i_{!} i^{!}\right) a_{p}^{!}$, which shows that $a_{p+1}^{!} f=0=i_{p+1}^{!} f \Rightarrow a_{p}^{!} f=0$. Hence by induction $i_{p}^{!} f=0 \forall p$ implies $f=a_{0}^{!} f=0$.

3.5. Localization of $\mathcal{O}^{\mathfrak{q}}$. Let $\mathfrak{g} \supset \mathfrak{q} \supset \mathfrak{b}$ be as in the introduction and let $G \supset Q \supset$ $B$ be the corresponding affine connected algebraic groups. We put $\mathcal{S}_{Q}=\mathcal{S}_{\mathfrak{q}}$ and hence $\mathcal{W}^{Q}=\mathcal{W}^{\mathfrak{q}}$, etc. Let $\mathcal{P}_{B}(G / Q) \subset \mathcal{P}(G / Q)$ be all perverse sheaves which are smooth along $B$-orbits. So the simple objects of $\mathcal{P}_{B}(G / Q)$ are just the intersection cohomology complexes $\mathcal{L}_{x}^{Q}$ of the closures of the $B$-orbits $B x Q / Q$, for $x \in \mathcal{W}^{Q}$. We want to prove

Proposition 3.5.1. There exists an equivalence of categories $\mathcal{O}^{\mathfrak{q}} \cong \mathcal{P}_{B}(G / Q)$ such that $L_{x}^{\mathfrak{q}}$ corresponds to $\mathcal{L}_{x}^{Q}$.

In fact, we will even construct an equivalence of triangulated categories from $D^{b}\left(\mathcal{O}^{\mathfrak{q}}\right)$ to the full subcategory $\mathcal{D}_{B}(G / Q) \subset \mathcal{D}(G / Q)$ consisting of all objects which are smooth along $B$-orbits. Here the case $\mathfrak{q}=\mathfrak{b}$ is localization [BB81].

Proposition 3.5.2. There exists an equivalence of triangulated categories $L^{B}$ : $D^{b}\left(\mathcal{O}^{\mathfrak{b}}\right) \cong \mathcal{D}_{B}(G / B)$ such that the obvious $t$-structure on the left corresponds to the perverse $t$-structure on the right and the induced equivalence $\mathcal{O}^{\mathfrak{b}} \cong \mathcal{P}_{B}(G / B)$ maps $L_{x}^{\mathfrak{b}}$ to $\mathcal{L}_{x}^{B}$ for all $x \in \mathcal{W}$.

Proof. For example [BB81, Soe86] and [Soe89a, Proposition 6].

We will now deduce the general case. Let $\pi: G / B \rightarrow G / Q$ be the projection and $d=\operatorname{dim}(Q / B)$ its fibre dimension.

Theorem 3.5.3. There exists an equivalence $L^{Q}: D^{b}\left(\mathcal{O}^{q}\right) \cong \mathcal{D}_{B}(G / Q)$ of triangulated categories such that the obvious t-structure on the left corresponds to the perverse $t$-structure on the right and the induced equivalence $\mathcal{O}^{q} \cong \mathcal{P}_{B}(G / Q)$ maps $L_{x}^{\mathfrak{q}}$ to $\mathcal{L}_{x}^{Q}$ for all $x \in \mathcal{W}^{Q}$. Furthermore, the diagram

$$
\begin{array}{ccc}
D^{b}\left(\mathcal{O}^{\mathfrak{q}}\right) & \cong & \mathcal{D}_{B}(G / Q) \\
\downarrow & \downarrow \pi^{!}[-d] \\
D^{b}\left(\mathcal{O}^{\mathfrak{b}}\right) \cong & \mathcal{D}_{B}(G / B)
\end{array}
$$

commutes up to natural equivalence, and its vertical arrows are faithful functors.

Proof [Theorem]. We procede in several steps.

Step 1. We show that $\pi^{!}: \mathcal{D}(G / Q) \rightarrow \mathcal{D}(G / B)$ is faithful. More precisely, we prove

Lemma 3.5.4. There exist natural isomorphisms $\pi_{!} \pi^{!} \mathcal{F}=\bigoplus_{x \in \mathcal{W}_{Q}} \mathcal{F}[2 l(x)]$ for $\mathcal{F} \in \mathcal{D}(G / Q)$.

Proof. By the decomposition theorem $\pi_{!} G / B \cong \bigoplus_{x \in \mathcal{W}_{Q}} \underline{G / Q}[-2 l(x)]$. Choose such an isomorphism. Then we get

$$
\begin{aligned}
\pi_{!} \pi^{!} \mathcal{F} & =\pi_{*} \mathcal{R H o m}\left(G / B, \pi^{!} \mathcal{F}\right) \\
& =\mathcal{R} \mathcal{H o m}\left(\pi_{!} \overline{G / B}, \mathcal{F}\right) \\
& =\bigoplus_{x \in \mathcal{W}_{Q}} \overline{\mathcal{F}[2 l(x)]}
\end{aligned}
$$

where the second step is Verdier duality.

Step 2. Let $\mathcal{P}_{Q^{-1}}(G / B)$ be the category of all perverse sheaves on $G / B$ which are smooth along the strata $B x Q / B$. The simple objects of this category are the $\mathcal{L}_{x}^{B}$ with $x \in \mathcal{W}^{Q}$. 
Proposition 3.5.5. The functors $\pi^{!}[-d]$ and $H^{d} \pi !$ define an equivalence of categories $\mathcal{P}_{Q^{-1}}(G / B) \cong \mathcal{P}_{B}(G / Q)$ such that $\mathcal{L}_{x}^{B}$ corresponds to $\mathcal{L}_{x}^{Q}$.

Proof [Proposition]. Let us first show how $H^{d} \pi_{!}: \mathcal{P}(G / B) \rightarrow \mathcal{P}(G / Q)$ is the right adjoint of $\pi^{!}[-d]: \mathcal{P}(G / Q) \rightarrow \mathcal{P}(G / B)$. Indeed for $\mathcal{G} \in \mathcal{P}(G / B), \mathcal{F} \in \mathcal{P}(G / Q)$ we have

$$
\begin{aligned}
\operatorname{Hom}_{\mathcal{D}}\left(\mathcal{G}, \pi^{!}[-d] \mathcal{F}\right) & =\operatorname{Hom}_{\mathcal{D}}\left(\pi_{!}[d] \mathcal{G}, \mathcal{F}\right) \\
& =\operatorname{Hom}_{\mathcal{D}}\left(H^{d} \pi ! \mathcal{G}, \mathcal{F}\right)
\end{aligned}
$$

the latter equality follows since we know that $\pi_{!}[d] \mathcal{G}$ is concentrated in perverse degrees $\leq 0$. Next we show that for any $\mathcal{F} \in \mathcal{P}(G / Q)$ the adjointness map going from $\left(H^{\bar{d}} \pi_{!}\right)\left(\pi^{!}[-d]\right) \mathcal{F}$ to $\mathcal{F}$ is an isomorphism. Indeed, this follows immediately from the preceding lemma. Now we need

Lemma 3.5.5. The functor $H^{d} \pi_{!}: \mathcal{P}_{Q^{-1}}(G / B) \rightarrow \mathcal{P}_{B}(G / Q)$ is exact.

Proof. It is sufficient to show that for all $\mathcal{G} \in \mathcal{P}_{Q^{-1}}(G / B)$ and all $i$ of parity different from $d$ we have $H^{i} \pi ! \mathcal{G}=0$. We may even assume $\mathcal{G}$ simple, say $\mathcal{G}=\mathcal{L}_{x}$, $x \in \mathcal{W}^{Q}$. But then $\mathcal{G} \cong \pi^{!}[-d] \mathcal{L}_{x}^{Q}$, and parity vanishing follows from the preceding lemma.

Finally we show that for all $\mathcal{G} \in \mathcal{P}_{Q^{-1}}(G / B)$ the adjointness map going from $\mathcal{G}$ to $\left(\pi^{!}[-d]\right)\left(H^{d} \pi_{!}\right) \mathcal{G}$ is an isomorphism. This is clear for all $\mathcal{G}$ of the form $\pi^{!}[-d] \mathcal{F}$, in particular for all simple $\mathcal{G}$. Then it follows for all $\mathcal{G}$ from the five lemma. The proposition is proven.

Step 3. It is evident that the equivalence $\mathcal{O}^{\mathfrak{b}} \cong \mathcal{P}_{B}(G / B)$ of Proposition 3.5.2 induces an equivalence $\mathcal{O}^{\mathfrak{q}} \cong \mathcal{P}_{Q^{-1}}(G / B)$. Together with the preceding proposition we get now a diagram of exact functors

$$
\begin{gathered}
\mathcal{O}^{\mathfrak{q}} \cong \mathcal{P}_{B}(G / Q) \\
\downarrow \\
\mathcal{O}^{\mathfrak{b}} \cong \mathcal{P}_{B}(G /[-d] \\
\pi^{!}[-d]
\end{gathered}
$$

that commutes up to natural equivalence. Certainly we can go over to bounded derived categories and obtain a diagram

$$
\begin{array}{ccc}
D^{b}\left(\mathcal{O}^{\mathfrak{q}}\right) & \cong & D^{b}\left(\mathcal{P}_{B}(G / Q)\right) \\
\downarrow & \downarrow \pi^{!}[-d] \\
D^{b}\left(\mathcal{O}^{\mathfrak{b}}\right) & \cong D^{b}\left(\mathcal{P}_{B}(G / B)\right) .
\end{array}
$$

So to prove the theorem we have only to establish a commutative diagram

$$
\begin{array}{ccc}
D^{b}\left(\mathcal{P}_{B}(G / Q)\right) & \longrightarrow & \mathcal{D}_{B}(G / Q) \\
\pi^{!}[-d] \downarrow & & \downarrow \pi \cdot[-d] \\
D^{b}\left(\mathcal{P}_{B}(G / B)\right) & \longrightarrow & \mathcal{D}_{B}(G / B)
\end{array}
$$

such that the horizontal arrows are equivalences. For the definition of these horizontal arrows, remark that for smooth $X$ we may interpret $\mathcal{P}(X)$ as a category of $\mathcal{D}$-modules and $\mathcal{D}(X)$ as a category of complexes of $\mathcal{D}$-modules, and get in this way an evident extension of the embedding $\mathcal{P}(X) \rightarrow \mathcal{D}(X)$ to an exact functor $D^{b}(\mathcal{P}(X)) \rightarrow \mathcal{D}(X)$. From this point of view it is clear how to define the horizontal arrows, and it is equally clear that the diagram commutes. We then apply Corollary 3.3.2 to see that the horizontal functors are equivalences. The theorem is proved. 
We now want to discuss standard objects. Recall the parabolic Verma modules $M_{x}^{\mathfrak{q}} \in \mathcal{O}^{\mathfrak{q}}$ from the introduction, and let $\mathcal{M}_{x}^{Q}=j_{x !} \mathcal{C}_{x} \in \mathcal{P}_{B}(G / Q)$ be the standard objects.

Proposition 3.5.7. Our equivalence $\mathcal{O}^{\mathfrak{q}} \cong \mathcal{P}_{B}(G / Q)$ identifies $M_{x}^{\mathfrak{q}}$ and $\mathcal{M}_{x}^{Q}$.

Proof. This is clear, since $M_{x}^{\mathfrak{q}}$ is the projective cover of $L_{x}^{\mathfrak{q}}$ in $\mathcal{O}_{\leq x}^{\mathfrak{q}}$ and $\mathcal{M}_{x}^{Q}$ is the projective cover of $\mathcal{L}_{x}^{Q}$ in $\mathcal{P}_{B}(G / Q)_{\leq x}$ (see section 3.2 for the notation).

Remarks. (1) The proposition allows us to avoid the use of sections 3.2 and 3.3 in the proof of the theorem. Namely, the only thing we used was Corollary 3.3.2 for the stratified space $G / Q$, and to prove this corollary we only needed that $\mathcal{P}_{B}(G / Q)$ has (1) finite homological dimension, (2) enough projectives and injectives, and that (3) each projective (resp. injective) has a filtration with standard (resp. costandard) subquotients. But (1)-(3) are known for $\mathcal{O}^{\mathfrak{q}}$, hence they follow for the equivalent category $\mathcal{P}_{B}(G / Q)$.

(2) Let us mention that the theorem allows us to interpret the derived functors of the left and right adjoint of the inclusion $\mathcal{O}^{\mathfrak{q}} \rightarrow \mathcal{O}^{\mathfrak{b}}$ geometrically as the left and right adjoints of $\pi^{!}[-d]$, i.e. as $\pi_{!}[d]$ and $\pi_{*}[-d]$. This in turn allows us to compute the effect of these "derived parabolic truncation functors" on simple objects.

3.6. Extensions of simple objects in $\mathcal{O}^{\mathfrak{q}}$. To any two elements $x, y$ of the Weyl group, Kazhdan and Lusztig [KL80a] associate the so-called KL-polynomial $P_{x, y}(q) \in \mathbb{Z}[q]$. Furthermore, they prove [KL80b] that the intersection cohomology matrix $I C(G / Q, t)$ of the stratified space $G / Q=\dot{\bigcup}_{w \in \mathcal{W} Q} B w Q / Q$ can be expressed in terms of their polynomials as

$$
I C(G / Q, t)_{x, y}=P_{x, y}\left(t^{-2}\right) t^{l(y)-l(x)} \quad \forall x, y \in \mathcal{W}^{Q} .
$$

Let us denote this matrix by $P^{Q}=P^{Q}(t)$. Remember now our algebra

$$
A_{Q}=\operatorname{Ext}_{\mathcal{O}_{\mathfrak{q}}}^{\bullet}\left(\oplus L_{x}^{\mathfrak{q}}, \oplus L_{x}^{\mathfrak{q}}\right)
$$

from section 1.3.

Proposition 3.6.1. The Hilbert polynomial of $A_{Q}$ is $P\left(A_{Q}, t\right)=\left(P^{Q}\right)^{T} P^{Q}$.

Proof. Indeed,

$$
\begin{aligned}
P\left(A_{Q}, t\right)_{x, y} & =\sum_{i} t^{i} \operatorname{dim} \operatorname{Ext}_{\mathcal{O}_{\mathfrak{q}}}^{i}\left(L_{y}^{\mathfrak{q}}, L_{x}^{\mathfrak{q}}\right) \text { by definition } \\
& =\sum_{i} t^{i} \operatorname{dim} \operatorname{Hom}_{\mathcal{D}}^{i}\left(\mathcal{L}_{y}^{Q}, \mathcal{L}_{x}^{Q}\right) \text { by the preceding section } \\
& =\left(\left(P^{Q}\right)^{T} P^{Q}\right)_{x, y} \text { by Theorem 3.4.1. } \square
\end{aligned}
$$

3.7. Parabolic-singular duality. We want to establish the first isomorphism of Theorem 1.1.3.

Theorem 3.7.1. Let $\lambda, \mathfrak{q}$ be as in the introduction and suppose $\mathcal{S}_{\lambda}=\mathcal{S}_{\mathfrak{q}}$. Then there is an isomorphism of $\mathbb{C}$-algebras

$$
\operatorname{End}_{\mathcal{O}_{\lambda}}(\oplus P(x \cdot \lambda)) \cong \operatorname{Ext}_{\mathcal{O}_{\mathfrak{q}}}^{\bullet}\left(\oplus L_{x}^{\mathfrak{q}}, \oplus L_{x}^{\mathfrak{q}}\right)
$$

such that the obvious idempotents $1_{x}$ on both sides correspond.

Proof. We will describe both sides in a combinatorial way to see that they are the same. In the case $\mathfrak{q}=\mathfrak{b}$ this is done in [Soe90]. We will now reduce our more general situation to this case. 
Step 1. The dimensions of both sides coincide.

Let $(P(x \cdot \lambda): M(z \cdot \lambda))$ be the Verma flag multiplicities. They are related to Jordan-Hölder-multiplicities by the so-called BGG-reciprocity formula

$$
(P(x \cdot \lambda): M(z \cdot \lambda))=[M(z \cdot \lambda): L(x \cdot \lambda)] .
$$

Now we calculate

$$
\begin{aligned}
& \operatorname{dim} \operatorname{End}_{\mathcal{O}_{\lambda}}(\bigoplus P(x \cdot \lambda))=\sum_{x, y \in \mathcal{W}^{Q}}[P(x \cdot \lambda): L(y \cdot \lambda)] \\
& =\sum_{x, y, z \in \mathcal{W}^{Q}}(P(x \cdot \lambda): M(z \cdot \lambda))[M(z \cdot \lambda): L(y \cdot \lambda)] \\
& =\sum_{x, y, z \in \mathcal{W}^{Q}}[M(z \cdot \lambda): L(x \cdot \lambda)][M(z \cdot \lambda): L(y \cdot \lambda)] \\
& =\sum_{x, y, z \in \mathcal{W}^{Q}} P_{z, x}(1) P_{z, y}(1) \text { by the KL-conjectures }
\end{aligned}
$$

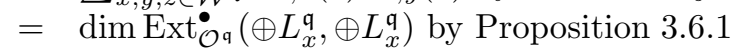

Step 2. Combinatorial description of the left hand side.

Let $S=S(\mathfrak{h})$ be the symmetric algebra over $\mathfrak{h}, C=C(\mathfrak{h})=S /\left(S^{+}\right)^{\mathcal{W}} S$ the coinvariants for the action of the Weyl group, and $C_{\lambda}=C_{\lambda}(\mathfrak{h})=C^{\mathcal{W}_{\lambda}}$ the $\mathcal{W}_{\lambda^{-}}$ invariants in $C$. Let $P_{\lambda}=P\left(w_{\circ} \cdot \lambda\right) \in \mathcal{O}_{\lambda}$ be a projective cover of the simple Verma module. If $\theta^{\text {out }}: \mathcal{O}_{\lambda} \rightarrow \mathcal{O}_{0}$ is the translation out of the walls, we have $\theta^{\text {out }} P_{\lambda} \cong P_{0}$. In [Soe90, Ber90] one may find the construction of isomorphisms $C_{\lambda}=$ $\operatorname{End}_{\mathcal{O}} P_{\lambda}$. Under these isomorphisms the map $\operatorname{End}_{\mathcal{O}} P_{\lambda} \rightarrow \operatorname{End}_{\mathcal{O}} \theta^{\text {out }} P_{\lambda}=\operatorname{End}_{\mathcal{O}} P_{0}$ corresponds to the inclusion $C_{\lambda} \hookrightarrow C_{0}=C$. The isomorphisms $C_{\lambda}=\operatorname{End}_{\mathcal{O}} P_{\lambda}$ give rise to the functors $\mathbb{V}=\mathbb{V}_{\lambda}=\operatorname{Hom}_{\mathcal{O}}\left(P_{\lambda}, \quad\right): \mathcal{O}_{\lambda} \rightarrow C_{\lambda}$-Mof. In [Soe90] it is proved that $\mathbb{V}$ induces isomorphisms $\operatorname{Hom}_{\mathcal{O}}(P(x \cdot \lambda), P(y \cdot \lambda)) \rightarrow \operatorname{Hom}_{C_{\lambda}}(\mathbb{V} P(x \cdot \lambda), \mathbb{V} P(y \cdot \lambda))$ for all $x, y \in \mathcal{W}^{\lambda}$. Thus $\operatorname{End}_{\mathcal{O}_{\lambda}}(\oplus P(x \cdot \lambda)) \cong \operatorname{End}_{C_{\lambda}}(\oplus \mathbb{V} P(x \cdot \lambda))$.

Step 3. Combinatorial description of the right hand side.

Analogously to $C_{\lambda}=C_{\lambda}(\mathfrak{h})$ one may define $C_{\mathfrak{q}}=C_{\mathfrak{q}}\left(\mathfrak{h}^{*}\right)$. Define a grading on $C_{\mathfrak{q}}$ by requiring that $\mathfrak{h}^{*}$ has degree 2. In [BGG73] one may find the construction of an isomorphism of graded $\mathbb{C}$-algebras $C_{\mathfrak{q}} \cong H^{\bullet}(G / Q)$, the cohomology ring of $G / Q$. Under this isomorphism the pull-back $H^{\bullet}(G / Q) \rightarrow H^{\bullet}(G / B)$ corresponds to the inclusion $C_{\mathfrak{q}} \hookrightarrow C_{\mathfrak{b}}=C$. In this way the hypercohomology gives rise to a functor $\mathbb{H}^{\bullet}: \mathcal{D}(G / Q) \rightarrow C_{\mathfrak{q}}$-mof. By Proposition 3.4.2 hypercohomology induces injections

$$
\operatorname{Ext}_{\mathcal{D}}^{\bullet}\left(\mathcal{L}_{x}^{Q}, \mathcal{L}_{y}^{Q}\right) \rightarrow \operatorname{Hom}_{C_{\mathfrak{q}}}\left(\mathbb{H}^{\bullet} \mathcal{L}_{x}^{Q}, \mathbb{H}^{\bullet} \mathcal{L}_{y}^{Q}\right)
$$

of graded vector spaces over $\mathbb{C}$. Theorem 3.5.3 gives isomorphisms

$$
\operatorname{Ext}_{\mathcal{O} \mathfrak{q}}^{\bullet}\left(L_{x}^{\mathfrak{q}}, L_{y}^{\mathfrak{q}}\right) \cong \operatorname{Ext}_{\mathcal{D}}^{\bullet}\left(\mathcal{L}_{x}^{Q}, \mathcal{L}_{y}^{Q}\right)
$$

Hence we have an injection $\operatorname{Ext}_{\mathcal{O}_{\mathfrak{q}}}^{\bullet}\left(\oplus L_{x}^{\mathfrak{q}}, \oplus L_{x}^{\mathfrak{q}}\right) \hookrightarrow \operatorname{End}_{C_{\mathfrak{q}}}\left(\oplus \mathbb{H}^{\bullet} \mathcal{L}_{x}^{Q}\right)$, which will soon turn out to be an isomorphism.

Step 4. The isomorphism.

Choose a $\mathcal{W}$-equivariant isomorphism $\mathfrak{h} \cong \mathfrak{h}^{*}$. Since $\mathcal{S}_{\lambda}=\mathcal{S}_{\mathfrak{q}}$, this gives an isomorphism $C_{\lambda} \cong C_{\mathfrak{q}}$. We just have to prove that there exist isomorphisms $\mathbb{V} P(x \cdot \lambda) \cong$ $\mathbb{H}^{\bullet} \mathcal{L}_{x}^{Q}$ in $C_{\lambda}$-Mod $\cong C_{\mathfrak{q}}$-Mod. (Remark that by Steps 1 and 2 this implies in particular that the injection at the end of the preceding step is indeed an isomorphism.) The existence of an isomorphism $\mathbb{V} P(x \cdot 0) \cong \mathbb{H} \bullet \mathcal{L}_{x}^{B}$ is established in [Soe90]. The general case then follows directly from

Lemma 3.7.2. $\quad$ (i) For all $x \in \mathcal{W}^{\lambda}$ there exists an isomorphism in $C_{\lambda}$-Mod

$$
\operatorname{res}_{C_{\lambda}}^{C} \mathbb{V}_{0} P(x \cdot 0) \cong \oplus_{z \in \mathcal{W}_{\lambda}} \mathbb{V}_{\lambda} P(x \cdot \lambda) \text {. }
$$


(ii) For all $x \in \mathcal{W}^{\mathfrak{q}}$ there exists an isomorphism in $C_{\mathfrak{q}}$-mod

$$
\operatorname{res}_{C_{\mathfrak{q}}}^{C} \mathbb{H}^{\bullet} \mathcal{L}_{x}^{B} \cong \oplus_{z \in \mathcal{W}_{\mathfrak{q}}}\left(\mathbb{H}^{\bullet} \mathcal{L}_{x}^{Q}\right)\langle 2 l(z)-d\rangle .
$$

Proof. (i) Let $\theta_{\text {on }}: \mathcal{O}_{0} \rightarrow \mathcal{O}_{\lambda}$ be the translation onto the walls. It is known that $\theta_{\text {on }} \theta^{\text {out }}$ is just a direct sum of $\left|\mathcal{W}_{\lambda}\right|$ copies of the identity functor on $\mathcal{O}_{\lambda}$. Now for all $x \in \mathcal{W}^{\lambda}$ we have $\theta^{\text {out }} P(x \cdot \lambda) \cong P(x \cdot 0)$, hence $\theta_{\text {on }} P(x \cdot 0) \cong \bigoplus_{z \in \mathcal{W}_{\lambda}} P(x \cdot \lambda)$. But, in addition, by the adjointness of the pair $\left(\theta^{\text {out }}, \theta_{\text {on }}\right)$

$$
\begin{aligned}
\mathbb{V}_{\lambda}\left(\theta_{o n} M\right) & =\operatorname{Hom}_{\mathcal{O}}\left(P_{\lambda}, \theta_{o n} M\right) \\
& =\operatorname{Hom}_{\mathcal{O}}\left(P_{0}, M\right) \\
& =\operatorname{res}_{C_{\lambda}}^{C}\left(\mathbb{V}_{0} M\right)
\end{aligned}
$$

for all $M \in \mathcal{O}_{0}$. Hence $\operatorname{res}_{C_{\lambda}}^{C} \mathbb{V}_{0} P(x \cdot 0) \cong \bigoplus_{z \in \mathcal{W}_{\lambda}} \mathbb{V}_{\lambda} P(x \cdot \lambda)$.

(ii) For all $x \in \mathcal{W}^{Q}$ we have

$$
\begin{aligned}
\operatorname{res}_{C_{\mathfrak{q}}}^{C} \mathbb{H} \bullet^{\bullet} \mathcal{L}_{x}^{B} & =\mathbb{H}^{\bullet} \pi_{*} \mathcal{L}_{x}^{B} \\
& =\mathbb{H}^{\bullet} \pi_{!} \pi^{!} \mathcal{L}_{x}^{Q}[-d] \\
& =\oplus_{z \in \mathcal{W}_{Q}} \mathbb{H}^{\bullet}\left(\mathcal{L}_{x}^{Q}[2 l(z)-d]\right) \\
& =\oplus_{z \in \mathcal{W}_{Q}}\left(\mathbb{H}^{\bullet} \mathcal{L}_{x}^{Q}\right)\langle 2 l(z)-d\rangle .
\end{aligned}
$$

The theorem is proved.

Recall now the ring $A^{Q}=\operatorname{Ext}_{\mathcal{O}}^{\bullet}(\oplus L(x \cdot \lambda), \oplus L(x \cdot \lambda))$.

Corollary 3.7.3. We have a ring isomorphism $A^{Q} \cong E\left(A_{Q}\right)$ such that the obvious idempotents $1_{x}$ on both sides correspond.

Proof. As explained in section 1.3, the theorem gives an equivalence of categories

$$
\mathcal{O}_{\lambda} \cong A_{Q} \text {-Mof }
$$

identifying $L(x \cdot \lambda) \in \mathcal{O}_{\lambda}$ with $A_{Q}^{0} 1_{x} \in A_{Q}$-Mof. From this we deduce an isomorphism of extension rings

$$
\operatorname{Ext}_{\mathcal{O}_{\lambda}}^{\bullet}(\oplus L(x \cdot \lambda), \oplus L(x \cdot \lambda)) \cong \operatorname{Ext}_{A_{Q}}^{\bullet}\left(A_{Q}^{0}, A_{Q}^{0}\right) \text {. }
$$

3.8. Canonicity of the grading on $\mathcal{O}_{\lambda}$. A very annoying feature of our paper is that at lots of places we just prove the existence of isomorphisms, instead of really constructing them. This might be an indication that we do not yet have the correct point of view. We want to explain in this section a part of our constructions that is canonical. Namely, we construct (in the notation of the preceding section) a completely canonical equivalence of categories

$$
\operatorname{Mof}-\left(\operatorname{End}_{\mathcal{D}}^{\bullet}\left(\oplus \mathcal{L}_{x}^{Q}\right)\right) \rightarrow \mathcal{O}_{\lambda}
$$

This can be done as follows: First put $P_{\lambda}=\theta^{\text {out }} M(-\rho)$. This is a projective cover of the simple Verma module $M\left(w_{\circ} \cdot \lambda\right) \in \mathcal{O}_{\lambda}$ that doesn't depend on any choices, and we have canonically $\operatorname{End}_{\mathfrak{g}} P_{\lambda}=C_{\lambda}$. Then we identify $\mathfrak{h}$ with $\mathfrak{h}^{*}$ via the Killing form and obtain an identification $C_{\lambda} \cong C_{\mathfrak{q}}$. Via this identification the intersection cohomology groups $\mathbb{H} \bullet \mathcal{L}_{x}^{Q}$ of the closures of $B$-orbits in $G / Q$ can be considered as $C_{\lambda}$-modules, and we may form the $\mathfrak{g}$-modules

$$
P(x \cdot \lambda)=\operatorname{Hom}_{C_{\lambda}}\left(\mathbb{H}^{\bullet} \mathcal{L}_{x}^{Q}, P_{\lambda}\right)
$$

for each $x \in \mathcal{W}^{Q}$. With the same arguments as in the proof of [Soe90, Proposition $6]$, we see that these $P(x \cdot \lambda)$ are projective covers of the $L(x \cdot \lambda)$ in $\mathcal{O}_{\lambda}$. (However we cannot canonically construct surjections $P(x \cdot \lambda) \rightarrow L(x \cdot \lambda)$.) Let us put $P=$ 
$\bigoplus P(x \cdot \lambda)$. This is a completely canonical projective generator of $\mathcal{O}_{\lambda}$, and the obvious map

$$
\left(\operatorname{End}_{\mathcal{D}}^{\bullet}\left(\bigoplus \mathcal{L}_{x}^{Q}\right)\right) \rightarrow \operatorname{End}_{\mathfrak{g}} P
$$

is an isomorphism. Now $P$ has commuting structures as a left $\mathfrak{g}$-module and as a left $\operatorname{End}_{\mathfrak{g}} P$-module. For general reasons the functor

$$
\otimes_{\left(\operatorname{End}_{\mathfrak{g}} P\right)} P: \operatorname{Mof}-\left(\operatorname{End}_{\mathfrak{g}} P\right) \rightarrow \mathcal{O}_{\lambda}
$$

is an equivalence of categories, and via the above isomorphism this gives the soughtfor equivalence

$$
\operatorname{Mof}-\left(\operatorname{End}_{\mathcal{D}}^{\bullet}\left(\oplus \mathcal{L}_{x}^{Q}\right)\right) \rightarrow \mathcal{O}_{\lambda}
$$

3.9. The ring $A_{B}$ is Koszul. The "correct" proof of this fact is geometric and will be given in section 4 . Here we give two less conceptual but easier proofs via selfduality.

Theorem 3.9.1. $A_{B}$ is Koszul.

First Proof. By the numerical Koszulity criterion we only need to check the matrix equation $P\left(A_{B}, t\right) P\left(E\left(A_{B}\right),-t\right)=1$. Here $P\left(A_{B}, t\right)=\left(P^{B}\right)^{T} P^{B}$ by Proposition 3.6.1. To compute the Hilbert polynomial of $E\left(A_{B}\right)$ we use selfduality. Namely, the corollary above gives us an isomorphism $E\left(A_{B}\right) \cong A^{B}$, but since $\mathcal{O}^{\mathfrak{b}}=\mathcal{O}_{0}$ and $L_{x}^{\mathfrak{b}}=L\left(x^{-1} w_{\circ} \cdot 0\right)$ we have an identity $A_{B}=A^{B}$ with $1_{x}$ corresponding to $1_{x^{-1} w_{\circ}}$. So, up to a reindexing of our idempotents, $E\left(A_{B}\right)=A_{B}$, and hence if we define the $\mathcal{W} \times \mathcal{W}$-matrix $P_{B}=P_{B}(t)$ by $\left(P_{B}\right)_{x, y}=P_{w_{\circ} x^{-1}, w_{\circ} y^{-1}}^{B}$, then $P\left(E\left(A_{B}\right), t\right)=P_{B}^{T} P_{B}$. But now the inversion formulas for KL-polynomials from [KL80a] yield $P^{B}(t) P_{B}(-t)^{T}=1$. Hence

$$
P\left(A_{B}, t\right) P\left(E\left(A_{B}\right),-t\right)=\left(P^{B}(t)\right)^{T} P^{B}(t) P_{B}(-t)^{T} P_{B}(-t)=1 .
$$

Second Proof. This is due to Roman Bezrukavnikov. One deduces the theorem from the following

Lemma 3.9.2. Suppose $A$ is a positively graded ring over the semisimple ring $A_{0}=$ $k$. Suppose furthermore $A$ is an algebra over a field $F$ and $\operatorname{dim}_{F} A_{i}<\infty$ for $i=0,1$.

If there is an isomorphism of graded F-algebras $A \cong E(A)$, then $A$ is Koszul.

Proof. Remark first that for any $V \in k$-Mof we have $\operatorname{dim}_{F}\left(V^{*}\right)=\operatorname{dim}_{F} V$, for $V^{*}=\operatorname{Hom}_{k}(V, k)$ as in section 2.7. Remark next that if $M, N \in A$-mod are graded $A$-modules and $N_{i}=0$ for all but finitely many $i$, then

$$
\operatorname{Hom}_{A}(M, N)=\prod_{\nu} \operatorname{hom}_{A}(M, N\langle\nu\rangle)
$$

and even

$$
\operatorname{Ext}_{A}^{i}(M, N)=\prod_{\nu} \operatorname{ext}_{A}^{i}(M, N\langle\nu\rangle)
$$

Now consider $I=A_{>0}$ and the short exact sequence $I \hookrightarrow A \rightarrow k$. From this we get a commutative diagram

$$
\begin{array}{rlc}
\operatorname{Ext}_{A}^{1}(k, k) & = & \left(I / I^{2}\right)^{*} \\
\uparrow & \uparrow \\
\operatorname{ext}_{A}^{1}(k, k\langle 1\rangle) & = & A_{1}^{*}
\end{array}
$$

where the vertical arrows are inclusions. Now our selfduality assumption tells us that both rows have the same dimension over $F$, hence $\operatorname{Ext}_{A}^{1}(k, k)=\operatorname{ext}_{A}^{1}(k, k\langle 1\rangle)$ 
and $\left(I / I^{2}\right)=A_{1}$. But from $\left(I / I^{2}\right)=A_{1}$ it follows that $A$ is generated by $A_{1}$ over $k$; hence, using selfduality again, $E(A)$ is generated by $\operatorname{Ext}_{A}^{1}(k, k)=\operatorname{ext}_{A}^{1}(k, k\langle 1\rangle)$ over $k^{\text {opp }}$. This in turn implies $\operatorname{Ext}_{A}^{i}(k, k)=\operatorname{ext}_{A}^{i}(k, k\langle i\rangle)$ for all $i$, hence $A$ is Koszul by Proposition 2.1.3.

Now remark as before that there is an isomorphism of graded $\mathbb{C}$-algebras $A_{B} \cong$ $E\left(A_{B}\right)$. The theorem follows immediately from the lemma.

Remark. We can now run the first proof backwards and deduce the inversion formulas for Kazhdan-Lusztig polynomials from the selfduality of $A_{B}$.

3.10. The rings $A_{Q}$ and $A^{Q}$ are Koszul. Here our argument avoiding mixed geometry is really screwed-up. By a "Koszul grading" on a ring we mean a grading such that the corresponding graded ring is Koszul. Consider first $A^{\mathfrak{q}}=\operatorname{End}_{\mathcal{O}}\left(\oplus P_{x}^{\mathfrak{q}}\right)$.

Proposition 3.10.1. The ring $A^{\mathfrak{q}}$ can be given a Koszul grading.

Proof. Consider the equivalence of categories $\mathcal{O}^{\mathfrak{b}} \cong \operatorname{Mof}-A^{B}$. Since

$$
\mathcal{O}^{\mathfrak{q}}=\left\{M \in \mathcal{O}^{\mathfrak{b}} \mid\left[M: L_{x}^{\mathfrak{b}}\right] \neq 0 \Rightarrow x \in \mathcal{W}^{Q}\right\},
$$

this induces an equivalence of categories $\mathcal{O}^{\mathfrak{q}} \cong \operatorname{Mof}-\left(A^{B} / I_{Q}\right)$, where $I_{Q} \subset A^{B}$ is the two-sided ideal generated by all $1_{x}$ with $x \in \mathcal{W} \backslash \mathcal{W}^{Q}$. Since both $A^{B} / I_{Q}$ and $A^{\mathfrak{q}}$ are basic rings with the same category of right modules $\mathcal{O}^{\mathfrak{q}}$, we find that they are isomorphic.

To prove the proposition we will show that the graded $\operatorname{ring} A^{B} / I_{Q}$ is Koszul. So we need to show that extensions between pure objects of mof- $\left(A^{B} / I_{Q}\right)$ vanish unless our objects have the correct weight. But since we know already that $A^{B}$ is Koszul, it will be sufficient to show that the restriction functor $\operatorname{Mof}-\left(A^{B} / I_{Q}\right) \rightarrow \operatorname{Mof}-\left(A^{B}\right)$ induces injections on extensions. In other words, we have to show that the inclusion $\mathcal{O}^{\mathfrak{q}} \rightarrow \mathcal{O}^{\mathfrak{b}}$ induces injections on extensions. But this is just the "faithful"-part of Theorem 3.5.3.

Theorem 3.10.2. (1) The ring $A_{Q}$ is Koszul.

(2) The ring $A^{Q}$ is Koszul.

(3) We have $A^{\mathfrak{q}} \cong A^{Q}$.

Proof. (1) Under the equivalence of categories $\mathcal{O}^{\mathfrak{q}} \cong$ Mof- $A^{\mathfrak{q}}$ the objects $\bigoplus L_{x}^{\mathfrak{q}}$ and $A_{0}^{\mathfrak{q}}$ correspond, both being just the direct sum of "all" simples (i.e. taking one from each isomorphism class). Hence we get a ring isomorphism

$$
\operatorname{Ext}_{\mathcal{O}_{\mathfrak{q}}}^{\bullet}\left(\bigoplus L_{x}^{\mathfrak{q}}, \bigoplus L_{x}^{\mathfrak{q}}\right) \cong \operatorname{Ext}_{-A^{\mathfrak{q}}}^{\bullet}\left(A_{0}^{\mathfrak{q}}, A_{0}^{\mathfrak{q}}\right)
$$

which can be read as $A_{Q} \cong E\left(\left(A^{\mathfrak{q}}\right)^{\mathrm{opp}}\right)$. Hence by Proposition 2.2.1 and Theorem 2.10.2 the $\operatorname{ring} A_{Q}$ is Koszul.

(2) We already know that $A^{Q} \cong E\left(A_{Q}\right)$ from Corollary 3.7.3. Hence $A^{Q}$ is Koszul by Theorem 2.10.2.

(3) The isomorphism $A_{Q} \cong E\left(\left(A^{\mathfrak{q}}\right)^{\text {opp }}\right)$ leads to $E\left(A_{Q}\right) \cong\left(A^{\mathfrak{q}}\right)^{\text {opp }}$, by Theorem 2.10.2. But from Corollary 3.7.3 we deduce that $E\left(A_{Q}\right) \cong A^{Q}$, and we know that $A^{Q} \cong\left(A^{Q}\right)^{\text {opp }}$ by the existence of a duality on $\mathcal{O}_{\lambda}$. 
3.11. Graded representation categories and Koszul duality. Given $\lambda, \mathfrak{q}$ as in the introduction with $\mathcal{S}_{\lambda}=\mathcal{S}_{\mathfrak{q}}$, we constructed finite dimensional graded $\mathbb{C}$-algebras $A_{Q}, A^{Q}$ which are Koszul rings with degree zero part the semisimple ring $k(Q)=$ $\bigoplus_{x \in \mathcal{W}^{Q}} \mathbb{C} 1_{x}$. We furthermore obtain isomorphisms $\left(A_{Q}\right)^{\text {opp }}=A_{Q},\left(A^{Q}\right)^{\text {opp }}=A^{Q}$ over $k(Q)$ from the dualities on $\mathcal{O}_{\lambda}$, resp. $\mathcal{O}^{\mathfrak{q}}$, and in section 1.3 we deduced the following facts from Theorem 1.1.3 (whose proof is already complete):

(1) There exists an equivalence of $\mathbb{C}$-categories $A_{Q}$-Mof $\cong \mathcal{O}_{\lambda}$ identifying $A_{Q}^{0} 1_{x}$ with $L(x \cdot \lambda)$.

(2) There exists an equivalence of $\mathbb{C}$-categories $A^{Q}$-Mof $\cong \mathcal{O}^{\mathfrak{q}}$ identifying $A_{0}^{Q} 1_{x}$ with $L_{x}^{\mathfrak{q}}$.

(3) There exists an isomorphism $A_{Q}^{!} \cong A^{Q}$ of graded $\mathbb{C}$-algebras over $k(Q)$.

Let us put $\mathcal{O}_{Q}=A_{Q}$-mof. Forgetting the grading $v$ together with a choice of an equivalence as in (1) gives us an exact functor $v: \mathcal{O}_{Q} \rightarrow \mathcal{O}_{\lambda}$. We have $v(M\langle i\rangle)=v M$ for every $M \in \mathcal{O}_{Q}$, and for any $M, N \in \mathcal{O}_{Q}$ the obvious maps

$$
\bigoplus \operatorname{Ext}_{\mathcal{O}_{Q}}^{n}(M\langle i\rangle, N) \rightarrow \operatorname{Ext}_{\mathcal{O}_{\lambda}}^{n}(v M, v N)
$$

are isomorphisms. For $x \in \mathcal{W}^{Q}$ let us define the objects $L_{Q}^{x}=A_{Q}^{0} 1_{x}$ and $P_{Q}^{x}=$ $A_{Q} 1_{x}$ in $\mathcal{O}_{Q}$. The $L_{Q}^{x}$ are simple, and every simple object of $\mathcal{O}_{Q}$ is isomorphic to exactly one of the $L_{Q}^{x}\langle i\rangle$. The $P_{Q}^{x}$ are projective covers of the $L_{Q}^{x}$. Obviously $P_{Q}^{x}, L_{Q}^{x} \in \mathcal{O}_{Q}$ are lifts of $P(x \cdot \lambda), L(x \cdot \lambda) \in \mathcal{O}_{\lambda}$, i.e. $v P_{Q}^{x} \cong P(x \cdot \lambda), v L_{Q}^{x} \cong L(x \cdot \lambda)$. We also want to lift the Verma modules to $\mathcal{O}_{Q}$. To this end, note that $M(x \cdot \lambda)$ is the projective cover of $L(x \cdot \lambda)$ in the truncated category

$$
\left(\mathcal{O}_{\lambda}\right)_{\nless x}=\left\{M \in \mathcal{O}_{\lambda} \mid y<x \Rightarrow[M: L(y \cdot \lambda)]=0\right\} .
$$

This means that under our equivalence $\mathcal{O}_{\lambda} \cong A_{Q}$-Mof the Verma module $M(x \cdot \lambda)$ has to correspond to $\left(A_{Q} / I_{<x}\right) 1_{x}$, where $I_{<x} \subset A_{Q}$ is the two-sided ideal generated by all $1_{y}$ with $y<x$ in the Bruhat order. So let us just define $M_{Q}^{x}=$ $\left(A_{Q} / I_{<x}\right) 1_{x} \in \mathcal{O}_{Q}$. We also define a duality $d: \mathcal{O}_{Q} \rightarrow \mathcal{O}_{Q}$ on $\mathcal{O}_{Q}$ by the formula $d M=\operatorname{Hom}_{\mathbb{C}}(M, \mathbb{C})$, using the isomorphism $A_{Q}=A_{Q}^{\text {opp }}$. Then $d(M\langle i\rangle)=(d M)\langle-i\rangle$ and $d L_{Q}^{x} \cong L_{Q}^{x}$.

Analogously we define the category $\mathcal{O}^{Q}=A^{Q}$-mof, the "forgetting of the grading" $v: \mathcal{O}^{Q} \rightarrow \mathcal{O}^{\mathfrak{q}}$, and the lifts $P_{x}^{Q}, M_{x}^{Q}, L_{x}^{Q} \in \mathcal{O}^{Q}$ of $P_{x}^{\mathfrak{q}}, M_{x}^{\mathfrak{q}}, L_{x}^{\mathfrak{q}} \in \mathcal{O}^{\mathfrak{q}}$. Note that all of these are to a certain extent unique: Up to isomorphism there is a unique basic algebra $A^{\mathfrak{q}}$ describing the category $\mathcal{O}^{\mathfrak{q}}$. There are several possible Koszul gradings on $A^{\mathfrak{q}}$, but by Corollary 2.5.2 all of these give rise to a graded ring isomorphic to $A^{Q}$. And since by Lemma 2.5.3 the lifts of indecomposable objects are unique up to shift of grading and isomorphism, $L_{x}^{Q}$ can be characterized (up to isomorphism) as the unique lift of $L_{x}^{\mathfrak{q}}$ that is pure of weight zero, and then $P_{x}^{Q}, M_{x}^{Q}$ are the unique lifts of $P_{x}^{\mathfrak{q}}, M_{x}^{\mathfrak{q}}$ surjecting onto $L_{x}^{Q}$. Similar remarks apply to $\mathcal{O}_{Q}$. In this notation, we can state

Theorem 3.11.1. There exists a contravariant equivalence of triangulated categories over $\mathbb{C}$

$$
K d: D^{b}\left(\mathcal{O}_{Q}\right) \longrightarrow D^{b}\left(\mathcal{O}^{Q}\right)
$$

such that $P_{Q}^{x}, M_{Q}^{x}, L_{Q}^{x} \in \mathcal{O}_{Q}$ get mapped to $L_{x}^{Q}, M_{x}^{Q}, P_{x}^{Q} \in \mathcal{O}^{Q}$, for all $x \in \mathcal{W}^{Q}$, and $K d(M[n])=(K d(M))[-n], K d(M\langle n\rangle)=(K d(M))[n]\langle n\rangle$ for all $M$. 
Proof. We may define $K d$ as the composition

$$
D^{b}\left(\mathcal{O}_{Q}\right) \stackrel{d}{\longrightarrow} D^{b}\left(\mathcal{O}_{Q}\right)=D^{b}\left(A_{Q^{-m o f}}\right) \stackrel{K}{\longrightarrow} D^{b}\left(A_{Q^{-m o f}}\right) \cong D^{b}\left(A^{Q} \text {-mof }\right)=D^{b}\left(\mathcal{O}^{Q}\right) .
$$

Then the only assertion which doesn't follow directly from Theorem 2.12.6 and the definitions is the formula $K d M_{Q}^{x} \cong M_{x}^{Q}$. But this is readily deduced from the following characterizations of $M_{x}^{Q}$ and $M_{Q}^{x}$.

Lemma 3.11.2. $M_{x}^{Q}$ is-up to isomorphism-the unique object $M \in D^{b}\left(\mathcal{O}^{Q}\right)$ such that

(1) $\operatorname{Hom}\left(P_{y}^{Q}\langle i\rangle, M[n]\right) \neq 0 \Rightarrow y \leq x$.

(2) $\operatorname{Hom}\left(P_{x}^{Q}\langle i\rangle, M[n]\right)=\mathbb{C}$ if $i=n=0$, zero else.

(3) $\operatorname{Hom}\left(M\langle i\rangle, L_{y}^{Q}[n]\right) \neq 0 \Rightarrow y \geq x$.

(4) $\operatorname{Hom}\left(M\langle i\rangle, L_{x}^{Q}[n]\right)=\mathbb{C}$ if $i=n=0$, zero else.

Lemma 3.11.3. $M_{Q}^{x}$ is-up to isomorphism-the unique object $M \in D^{b}\left(\mathcal{O}_{Q}\right)$ such that

(1) $\operatorname{Hom}\left(P_{Q}^{y}\langle i\rangle, M[n]\right) \neq 0 \Rightarrow y \geq x$.

(2) $\operatorname{Hom}\left(P_{Q}^{x}\langle i\rangle, M[n]\right)=\mathbb{C}$ if $i=n=0$, zero else.

(3) $\operatorname{Hom}\left(M\langle i\rangle, L_{Q}^{y}[n]\right) \neq 0 \Rightarrow y \leq x$.

(4) $\operatorname{Hom}\left(M\langle i\rangle, L_{Q}^{x}[n]\right)=\mathbb{C}$ if $i=n=0$, zero else.

Proof. We will only prove the first lemma. Certainly $M=M_{x}^{Q}$ satisfies the stated properties. On the other hand suppose $M \in D^{b}\left(\mathcal{O}^{Q}\right)$ also satisfies (1) through (4). We deduce further properties of $M$.

(5) By (1) all cohomology groups $H^{\nu}(M)$ have only composition factors $L_{y}^{Q}(i)$ with $y \leq x$. By $(2)$ only $H^{0}(M)$ has a composition factor of the form $L_{x}^{Q}(i)$.

(6) From (5) and (3) we deduce that $H^{\nu}(M)=0$ for $\nu>0$. By (4) then $H^{0}(M)$ has the unique simple quotient $L_{x}^{Q}$.

(7) By (5) and (6) there is a surjection $M_{x}^{Q} \rightarrow H^{0}(M)$. By (5) and (6) again this can be lifted to a nonzero map $M_{x}^{Q} \rightarrow M$.

Now complete this map to a distinguished triangle $M_{x}^{Q} \rightarrow M \rightarrow E \stackrel{[1]}{\rightarrow}$. We have to show that $E=0$. Let $H^{\nu}(E)$ be the highest nonvanishing cohomology group. Then there is a nonzero map $E \rightarrow L_{y}^{Q}\langle i\rangle[\nu]$ for suitable $y, i$. But $y<x$ by (5) and construction, and $y \geq x$ by (3). This contradiction shows that $E=0$.

For any $M \in \mathcal{O}$ let $M=\operatorname{rad}^{0} M \supset \operatorname{rad}^{1} M \supset \ldots$ be the radical filtration and put $\overline{\mathrm{rad}}^{i} M=\operatorname{rad}^{i} M / \mathrm{rad}^{i+1} M$. Parts (i) and (ii) of the following theorem are to be seen as definition of the $n_{Q}^{x, y}(i), n_{x, y}^{Q}(i)$.

Theorem 3.11.4. (i)

$$
\begin{aligned}
& \operatorname{dim} \operatorname{Ext}_{\mathcal{O}}^{i}(M(x \cdot \lambda), L(y \cdot \lambda))=\left[\begin{array}{c}
\left.\operatorname{rad}^{i} M_{x}^{\mathfrak{q}}: L_{y}^{\mathfrak{q}}\right] \\
\|(3)
\end{array}\right. \\
& \operatorname{dim} \operatorname{Ext}_{\mathcal{O}_{Q}}^{i}\left(M_{Q}^{x}, L_{Q}^{y}\langle i\rangle\right) \quad \stackrel{(2)}{=}\left[M_{x}^{Q}: L_{y}^{Q}\langle i\rangle\right] \\
& n_{Q}^{x, y}(i) \quad=\left(P_{y}^{Q}: M_{x}^{Q}\langle i\rangle\right)
\end{aligned}
$$


(ii)

$$
\begin{array}{ccc}
\operatorname{dim} \operatorname{Ext}_{\mathcal{O q}\left(\left(M_{x}^{\mathfrak{q}}, L_{y}^{\mathfrak{q}}\right)\right.}^{i} & = & {\left[\overline{\operatorname{rad}}^{i} M(x \cdot \lambda): L(y \cdot \lambda)\right]} \\
\operatorname{dim} \operatorname{Ext}_{\mathcal{O}^{Q}}^{i}\left(M_{x}^{Q}, L_{y}^{Q}\langle i\rangle\right) & \stackrel{(6)}{=} & {\left[M_{Q}^{x}: L_{Q}^{y}\langle i\rangle\right]} \\
n_{x, y}^{Q}(i) & = & \left(P_{Q}^{y}: M_{Q}^{x}\langle i\rangle\right) .
\end{array}
$$

(iii) If we collect the $n$ 's into $\mathcal{W}^{Q} \times \mathcal{W}^{Q}$-matrices $P^{Q}(t), P_{Q}(t)$ with entries in $\mathbb{Z}[t]$ given by $\left(P^{Q}(t)\right)_{x, y}=\sum n_{x, y}^{Q}(i) t^{i},\left(P_{Q}(t)\right)_{x, y}=\sum n_{Q}^{x, y}(i) t^{i}$, then the matrix equation

$$
\left(P_{Q}(t)\right)^{T} P^{Q}(-t)=1
$$

holds (where the upper index $T$ transposes the matrix).

(iv) In terms of the Kazhdan-Lusztig polynomials $P_{x, y}$ defined in [KL80a] we have

$$
\begin{aligned}
& \left(P^{Q}(t)\right)_{x, y}=P_{x, y}\left(t^{-2}\right) t^{l(y)-l(x)}, \\
& \left(P_{Q}(t)\right)_{x, y}=\sum_{z \in \mathcal{W}_{Q}}(-1)^{l(z)} P_{x z w_{0}, y w_{0}}\left(t^{-2}\right) t^{l(x)-l(y)}
\end{aligned}
$$

for all $x, y \in \mathcal{W}^{Q}$. Our polynomials are of degree $l(y)-l(x)$ and $l(x)-l(y)$ respectively with leading coefficient one.

Remark. The equalities (1) (resp. (5)) mean that extensions of Vermas by simples are "pure", i.e. $\operatorname{Ext}_{\mathcal{O}_{Q}}^{i}\left(M_{Q}^{x}, L_{Q}^{y}\langle n\rangle\right)=0$ unless $i=n$ (resp. ...). This means that our graded Vermas are "Koszul modules" in the terminology of section 2.14 .

Proof. (i) We start with (3). We deduce from Proposition 2.4.1 that

$$
\left[\overline{\operatorname{rad}}^{i} M_{x}^{\mathfrak{q}}: L_{y}^{\mathfrak{q}}\right]=\left[M_{x}^{Q}: L_{y}^{Q}\langle i\rangle\right] .
$$

Next we prove (1) and (2) in one argument. Namely,

$$
\begin{aligned}
\operatorname{Ext}_{\mathcal{O}_{Q}}^{i}\left(M_{Q}^{x}, L_{Q}^{y}\langle n\rangle\right) & =\operatorname{Hom}_{D^{b}\left(\mathcal{O}_{Q}\right)}\left(M_{Q}^{x}, L_{Q}^{y}\langle n\rangle[i]\right) \\
& =\operatorname{Hom}_{D^{b}\left(\mathcal{O}^{Q}\right)}\left(P_{y}^{Q}, M_{x}^{Q}[i-n]\langle-n\rangle\right) .
\end{aligned}
$$

But clearly this vanishes if $i \neq n$, and in the case $i=n$ it has dimension $\left[M_{x}^{Q}\right.$ : $\left.L_{y}^{Q}\langle i\rangle\right]$.

To clarify the structure of the following arguments let us develop some generalities. For any abelian category $\mathcal{A}$ let $[\mathcal{A}]$ be its Grothendieck group. Any $A \in \mathcal{A}$ gives $[A] \in[\mathcal{A}]$. We often just write $A$ instead of $[A]$.

Now consider the Grothendieck group $\left[\mathcal{O}^{Q}\right]$ of $\mathcal{O}^{Q}$. We let $\mathbb{Z}\left[t, t^{-1}\right]$ act on it via $t[M]=[M\langle 1\rangle]$. Then it becomes a free $\mathbb{Z}\left[t, t^{-1}\right]$-module of rank $\left|\mathcal{W}^{Q}\right|$ with three bases $\left[P_{x}^{Q}\right],\left[M_{x}^{Q}\right]$ and $\left[L_{x}^{Q}\right]$. Furthermore, $\left[\mathcal{O}^{Q}\right]$ comes with a symmetric $\mathbb{Z}\left[t, t^{-1}\right]$ bilinear form given by

$$
\langle M, N\rangle=\sum_{i, \nu}(-1)^{i} \operatorname{dim} \operatorname{Ext}_{\mathcal{O} Q}^{i}(M, d N\langle\nu\rangle) t^{\nu} .
$$

With respect to this form the $\left[P_{x}^{Q}\right]$ and the $\left[L_{x}^{Q}\right]$ are dual bases, whereas the $\left[M_{x}^{Q}\right]$ form an orthonormal basis. It is then clear that

$$
[M]=\sum_{z}\left\langle P_{z}^{Q}, M\right\rangle\left[L_{z}^{Q}\right]=\sum_{z}\left\langle M, M_{z}^{Q}\right\rangle\left[M_{z}^{Q}\right]
$$

for any $M \in \mathcal{O}^{Q}$. In other words, $\left\langle P_{z}^{Q}, M\right\rangle=\sum_{i}\left[M: L_{z}^{Q}\langle i\rangle\right] t^{i}$ and $\left\langle M, M_{z}^{Q}\right\rangle=$ $\sum_{i}\left(M: M_{z}^{Q}\langle i\rangle\right) t^{i}$, where the "virtual Verma flag multiplicities" $\left(M: M_{z}^{Q}\langle i\rangle\right)$ are 
just defined by $[M]=\sum_{z, i}\left(M: M_{z}^{Q}\langle i\rangle\right)\left[M_{z}^{Q}\langle i\rangle\right]$ and coincide with the actual Verma flag multiplicities in case $M$ has a Verma flag. Now (4) follows from

$$
\sum_{i}\left[M_{x}^{Q}: L_{y}^{Q}\langle i\rangle\right] t^{i}=\left\langle P_{y}^{Q}, M_{x}^{Q}\right\rangle=\sum_{i}\left(P_{y}^{Q}: M_{x}^{Q}\langle i\rangle\right) t^{i},
$$

and we have established (i).

(ii) is proved the same way.

(iii) Note that $\left(P_{Q}(t)\right)_{x, y}=\left\langle P_{y}^{Q}, M_{x}^{Q}\right\rangle$ and $\left(P^{Q}(-t)\right)_{x, y}=\left\langle M_{x}^{Q}, L_{y}^{Q}\right\rangle$. This reduces our statement to linear algebra.

(iv) This follows from the literature [Soe89a, Irv90, Irv88, CC87, Bar83, BB86, ES87]. The first equality is also a direct consequence of the results expounded in section 3.5. Namely, we can compute

$$
\begin{aligned}
\operatorname{dim} \operatorname{Ext}_{\mathcal{O} \mathfrak{q}}^{i}\left(M_{x}^{\mathfrak{q}}, L_{y}^{\mathfrak{q}}\right) & =\operatorname{dim} \operatorname{Hom}_{\mathcal{D}}^{i}\left(\mathcal{M}_{x}^{Q}, \mathcal{L}_{y}^{Q}\right) \text { by section } 3.5 \\
& =\operatorname{dim} \operatorname{Hom}_{\mathcal{D}}^{i}\left(j_{x !} \mathcal{C}_{x}, \mathcal{I} \mathcal{C}_{y}\right) \text { in other notation } \\
& =\operatorname{dim} \operatorname{Hom}_{\mathcal{D}}^{i}\left(\mathcal{C}_{x}, j_{x}^{!} \mathcal{I} \mathcal{C}_{y}\right) \text { by adjointness }
\end{aligned}
$$

and this certainly is a suitable coefficient of $I C(G / Q, t)_{x, y}=\left(P^{Q}(t)\right)_{x, y}$.

We also want to show that our graded versions of projectives admit graded Verma flags. In the case of $\mathcal{O}_{Q}$ this follows directly from the following proposition, whose proof is stolen from [Jan87, II, 4.16]:

Proposition 3.11.5. Let $M \in \mathcal{O}_{Q}$ be such that $\operatorname{Ext}_{\mathcal{O}_{Q}}^{1}\left(M, d M_{Q}^{x}\langle j\rangle\right)=0$ for all $x, j$. Then $M$ has a filtration with subquotients $M_{Q}^{y}\langle j\rangle$.

Proof. Without restriction we may suppose $M \neq 0$. Choose $y \in \mathcal{W}$ maximal such that $\operatorname{Hom}\left(M, L_{Q}^{y}\langle i\rangle\right) \neq 0$ for some $i$. It follows that $\operatorname{Hom}\left(M, L_{Q}^{z}\langle j\rangle\right)=0$ for all $z>y$, all $j$. From the assumptions we know in particular that $\operatorname{Ext}^{1}\left(M, d M_{Q}^{z}\langle j\rangle\right)=0$ for all $z>y$, all $j$. These two statements together give $\operatorname{Ext}^{1}\left(M, L_{Q}^{z}\langle j\rangle\right)=0$ for all $z>y$, all $j$, and hence $\operatorname{Ext}^{1}\left(M, \operatorname{rad} M_{Q}^{y}\langle i\rangle\right)=0$. Thus any nonzero morphism $M \rightarrow L_{Q}^{y}\langle i\rangle$ lifts to a surjection $M \rightarrow M_{Q}^{y}\langle i\rangle$. But the kernel of this surjection again satisfies the conditions of the proposition, and we are done by induction.

The case of $\mathcal{O}^{Q}$ is similar. As another direct consequence of our results we obtain a new proof of the following result from [Irv88, Bar83, BB86].

Proposition 3.11.6. Verma modules are rigid, i.e. for any $\mu \in \mathfrak{h}^{*}$ the socle and radical filtrations on $M(\mu)$ coincide.

Proof. We know that $M(\mu)$ has simple socle and simple top. By some equivalence of categories [Soe90] we may suppose $\mu$ is integral, say $\mu=x \cdot \lambda$. Write $M(x \cdot \lambda)=v M_{Q}^{x}$. By Proposition 2.4 .1 both the socle and radical filtration on $M(x \cdot \lambda)$ correspond to the grading filtration on $M_{Q}^{x}$.

Remark. The same argument proves that projectives in $\mathcal{O}$ with a simple socle are rigid. This applies in particular to the projective covers of simple Verma modules. In fact, the argument proves that any object in $\mathcal{O}^{\mathfrak{q}}$ or $\mathcal{O}_{\lambda}$ that has simple socle and top and admits a graded version is rigid.

Continuing in the spirit of the above results one might for two parabolics $P \subset$ $Q$ define graded versions of translation functors $\theta_{!}, \theta_{*}: \mathcal{O}_{P} \rightarrow \mathcal{O}_{Q}$ and $\theta^{!}, \theta^{*}$ : $\mathcal{O}_{Q} \rightarrow \mathcal{O}_{P}$. Their derived functors should correspond under Koszul duality to the 
graded derived versions of the embedding $\mathcal{O}^{\mathfrak{q}} \hookrightarrow \mathcal{O}^{\mathfrak{p}}$ and its adjoints, the "parabolic truncation functors". However we didn't work this out.

\section{Koszul RINGS ARISING FROM 'MIXED' GEOMETRY}

One knows that various categories of representations may be interpreted geometrically as categories of perverse sheaves. For example, the category $\mathcal{O}^{\mathfrak{q}}$ (for regular integral weight) is equivalent to the category $\mathcal{P}_{B}(G / Q)$ of perverse sheaves on $G / Q$ which are smooth along the Schubert stratification (see 3.5). In this section we show that the graded representation category $\mathcal{O}^{Q}$ may be identified with an appropriate full subcategory of the corresponding category $\mathcal{P}_{B}(G / Q)_{\text {mixed }}$ of mixed perverse sheaves. As a bonus, we also get a natural explanation of the Koszulity of $\mathcal{O}^{Q}$.

The categories of (mixed) sheaves do not arise naturally as the categories of modules over some algebra. In the preliminary sections we develop some category language to overcome this nuisance.

4.1. Mixed categories and graded algebras (see [BMS87, BGS88]). For simplicity we fix a ground field $F$ and assume that all categories are $F$-categories and all functors are $F$-linear. By an "artinian category" we mean an abelian category with objects of finite length. For an artinian category $\mathcal{A}$ we denote by $\operatorname{Irr} \mathcal{A}$ the set of isomorphism classes of irreducible objects of $\mathcal{A}$.

Definition 4.1.1. A mixed category is an artinian category $\mathcal{M}$ equipped with a map $w_{\mathcal{M}}=w: \operatorname{Irr} \mathcal{M} \rightarrow \mathbb{Z}$ (called a weight) such that for any two irreducible objects $M, N$ one has $\operatorname{Ext}^{1}(M, N)=0$ if $w(M) \leq w(N)$.

Let $(\mathcal{M}, w)$ be a mixed category. An object $M$ of $\mathcal{M}$ is called pure of weight $i$ if all its irreducible components have same weight $i$. Any such object is semisimple. Denote by $\mathcal{M}_{i}$ the full subcategory of pure objects of weight $i$. Clearly, $\mathcal{M}^{s s}=$ $\bigoplus \mathcal{M}_{i}$ is the full subcategory formed by all semisimple objects of $\mathcal{M}$.

Lemma 4.1.2. Any object $L \in \mathcal{M}$ has a unique finite increasing filtration $W_{\bullet}=$ $W_{\bullet} L$ such that $\operatorname{gr}_{i}^{W} L=W_{i} L / W_{i-1} L$ is a pure object of weight $i$ for all $i \in \mathbb{Z}$. Any morphism in $\mathcal{M}$ is strictly compatible with $W_{\bullet}$.

Proof. Left to the reader.

The filtration $W_{\bullet}$ is called a weight filtration. Note that any full subcategory $\mathcal{M}^{\prime} \subset$ $\mathcal{M}$ closed under direct sums and subquotients is itself a mixed category (with respect to $w_{\mathcal{M}^{\prime}}=\left.w_{\mathcal{M}^{\prime}}\right|_{\mathcal{M}}$ ). Given any set of objects $S$ in $\mathcal{M}$, we denote by $\mathcal{M}_{S}$ the smallest such subcategory that contains $S$.

Definition 4.1.3. A functor $\pi: \mathcal{M}^{(1)} \rightarrow \mathcal{M}^{(2)}$ between mixed categories is called pure if $\pi$ is exact and sends $\mathcal{M}_{i}^{(1)}$ to $\mathcal{M}_{i}^{(2)}$, for each $i \in \mathbb{Z}$.

For example, the above embedding $\mathcal{M}_{S} \hookrightarrow \mathcal{M}$ is pure, and any fully faithful pure embedding comes this way. The mixed categories form a 2-category with 1-morphisms being pure functors.

Examples. (1) The category $\mathcal{H}_{F}$ of polarizable $F$-Hodge structures is a mixed category (we assume $F \subset \mathbb{C}$ and take the usual weight $w$ ). More generally, the category $\mathcal{H}(X)$ of mixed Hodge modules on an algebraic variety $X$ over $\mathbb{C}$ is a mixed category (see [Sai90]). 
(2) The category $\mathcal{M}(X)$ of mixed $\mathbb{Q}_{l}$-sheaves on an algebraic variety $X$ over a finite field is not mixed. However, its full subcategory consisting of those perverse sheaves $\mathcal{F}$ with $\operatorname{gr}_{i}^{W} \mathcal{F}$ semisimple for all $i \in \mathbb{Z}$ is mixed. Unfortunately, one still does not know if this mixed category is stable with respect to various standard functors (e.g., if the action of the Frobenius on $H^{\bullet}\left(X, \mathbb{Q}_{l}\right)$ is semisimple, the so-called "standard conjectures").

Remark. The above examples explain the origin of the name mixed categories. The still conjectural categories of mixed motivic $F$-sheaves on an algebraic variety should also be mixed if $\operatorname{char} F=0$. Beware that if $\operatorname{char} F \neq 0$, then these categories are not mixed categories in the sense of 4.1.1.

Definition 4.1.4. For an integer $d$ a degree $d$ Tate twist on a mixed category $\mathcal{M}$ is an automorphism $\langle d\rangle$ of $\mathcal{M}$, written $M \mapsto M\langle d\rangle$, with the property that $w(M\langle d\rangle)=w(M)+d$ for all $M \in \operatorname{Irr} \mathcal{M}$.

Then for any $M$ one has $W_{i}(M\langle d\rangle)=\left(W_{i-d} M\right)\langle d\rangle$. For any $n \in \mathbb{Z}$ we denote by $\langle n d\rangle$ the $n$-th power of $\langle d\rangle$, so that $\langle-d\rangle$ is the inverse to $\langle d\rangle$ and $\langle n d\rangle \circ\langle m d\rangle=$ $\langle(m+n) d\rangle$. If $n \in \mathbb{Z}$, then $\langle n d\rangle$ is a degree $n d$ Tate twist. If $\mathcal{M}^{1}, \mathcal{M}^{2}$ are mixed categories equipped with Tate twists $\langle d\rangle^{i}$ of the same degree $d$, then for any pure functor $\phi: \mathcal{M}^{1} \rightarrow \mathcal{M}^{2}$ the functor $\langle d\rangle^{2} \circ \phi \circ\langle-d\rangle^{1}$ is also pure. We'll say that $\phi$ is compatible with Tate twists if we are given an isomorphism $\epsilon: \phi \cong\langle d\rangle^{2} \circ \phi \circ\langle-d\rangle^{1}$. Thus the mixed categories equipped with degree $d$ Tate twists form a 2-category with 1-morphisms being pairs $(\phi, \epsilon)$ as above. The mixed categories occurring in the above examples are equipped with a standard Tate twist of degree -2 . We write $\mathcal{F}\langle-2 n\rangle=\mathcal{F}(n)$.

Sometimes it is convenient to represent $\langle d\rangle$ as $d$-th power of a degree 1 Tate twist $\langle 1\rangle$. One may do this in a universal manner as follows. Put $\mathcal{M}^{1 / d}:=$ $\bigoplus_{0<a<d-1} \mathcal{M}^{(a)}$ (the sum of $d$ copies of $\mathcal{M}$ ). Denote by $\langle 1\rangle$ the automorphism of $\mathcal{M}^{1 / d}$ that shifts $\mathcal{M}^{(a)} \subset \mathcal{M}^{1 / d}$ to $\mathcal{M}^{(a+1)}$ for $a<d-1$, and coincides with $\mathcal{M}^{(d-1)}=\mathcal{M} \stackrel{\langle d\rangle}{\rightarrow} \mathcal{M}=\mathcal{M}^{(0)}$ on $\mathcal{M}^{(d-1)}$. Let us identify $\mathcal{M}$ with $\mathcal{M}^{(0)} \subset \mathcal{M}^{1 / d}$. Then $\mathcal{M}^{1 / d}$ is a mixed category with respect to the weight $w$ defined as $w(M\langle i\rangle)=$ $w(M)+i$ for $M \in \operatorname{Irr} \mathcal{M} \subset \operatorname{Irr} \mathcal{M}^{1 / d}, \mathcal{M} \subset \mathcal{M}^{1 / d}$ is a pure fully faithful embedding, and $\langle d \cdot 1\rangle$ coincides with $\langle d\rangle$ on $\mathcal{M}$.

The most trivial example of a mixed category with degree 1 Tate twist is the category $\operatorname{vect}_{F}$ of graded finite dimensional vector spaces over a field $F$. Although this is not necessary for us from a strictly logical point of view, let us show in the remainder of this subsection that any mixed $F$-category with Tate twist and finite dimensional Hom's is equivalent to the category of graded vector spaces equipped with certain extra symmetries.

Definition 4.1.5. A mixed algebra $A$ is a projective limit of a family of graded finite dimensional algebras $A_{\alpha}^{\bullet}$ such that $A_{\alpha}^{i}=0$ for $i<0$ and the $A_{\alpha}^{0}$ are semisimple $F$-algebras.

In other words, a mixed algebra $A=\prod A^{i}$ is a ring object in the abelian tensor category $\left.\varliminf_{\left(\text {vect }_{F}\right.}\right)$ of graded profinite dimensional vector spaces such that $A^{i}=0$ for $i<0$ and $A^{0}$ is a projective limit of semisimple algebras. For a mixed algebra $A$ we denote by $A$-mof the category of $F$-finite dimensional continuous $A$-modules, i.e. for $A=\underline{\lim }\left(A_{\alpha}\right)$ one has $A$-mof $=\bigcup_{\alpha} A_{\alpha}$-mof. If $M \in A$-mof is an irreducible module, then $A^{>0} M=0$, i.e. $M$ is actually an irreducible $A^{0}=A / A^{>0}$-module. 
It is supported in a single degree $i$, i.e. $M^{j}=0$ for $j \neq i$; set $w(M):=-i$. It is clear that ( $A$-mof, $w$ ) is a mixed category. The shift of degrees functor $M \mapsto$ $M\langle 1\rangle,(M\langle 1\rangle)^{i}=M^{i-1}$, is a degree 1 Tate twist on $A$-mof. Note that the category $\lim (A \text {-mof })_{0}$ (which is the category of all continuous non-graded $A^{0}$-modules) has

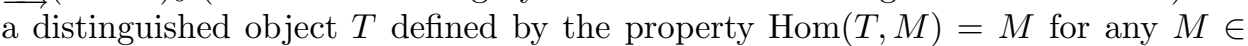
( $A$-mof $)_{0}$; clearly $T$ contains any irreducible object with finite non-zero multiplicity.

Proposition 4.1.6. The correspondence $A \mapsto(A$-mof, $w,\langle 1\rangle, T)$ is a one-to-one correspondence between mixed algebras and collections $(\mathcal{M}, w,\langle 1\rangle, T)$, where $(\mathcal{M}, w)$ is a mixed category, $\langle 1\rangle$ is a degree 1 Tate twist on $\mathcal{M}$, and $T$ is an object of $\lim _{\longrightarrow} \mathcal{M}_{0}$ such that $T$ contains any irreducible object of $\mathcal{M}_{0}$ with finite non-zero multiplicity.

Proof. Let us construct the inverse correspondence. Take $(\mathcal{M}, w,\langle 1\rangle, T)$ as above. To define the mixed algebra $A$ consider the functor $\phi: \mathcal{M} \rightarrow \operatorname{vect}_{F}, \phi(P)^{i}:=$ $\operatorname{Hom}\left(T, \operatorname{gr}_{0}^{w}(P\langle-i\rangle)\right)$. This is a pure faithful functor compatible with Tate twist, i.e. one has $\phi(M\langle a\rangle)^{i}=\phi(M)^{i-a}$. Consider the components $\phi^{i}: \mathcal{M} \rightarrow \operatorname{vect}_{F}$. The above compatibility shows that the space of morphisms of functors $\operatorname{Hom}\left(\phi^{a}, \phi^{a+i}\right)$ is independent of $a \in \mathbb{Z}$; denote it by $A^{i}$. Note that $A^{0}=\operatorname{End} T$ and $A^{i}=0$ for $i<0$. The composition of morphisms defines a product $A^{i} \times A^{j} \rightarrow A^{i+j}$, therefore $A=\prod A^{i}$ is a ring. It acts canonically on the graded vector space $\phi(P)$ for any $P \in \mathcal{M}$. Clearly $A$ coincides with the projective limit of its images $A_{P}$ in End $\phi(P)$. Therefore $A$ is a mixed algebra, and our $\phi$ may be considered as a pure functor $\mathcal{M} \rightarrow A$-mof compatible with Tate twists. It is easy to check that this is an equivalence of categories.

Exercises. (1) Describe the pure functors between the mixed categories in terms of the corresponding mixed algebras.

(2) Spell out the analog of the above proposition for arbitrary mixed categories (without Tate twists).

4.2. Projective objects in artinian categories. Below we'll use several times the following lemma of folklore. Its proof is left to the reader.

Let $\mathcal{C}$ be any artinian category. Denote by $\{L\}$ a collection of representatives of isomorphism classes of irreducible objects in $\mathcal{C}$, and put $F_{L}=\operatorname{End} L$. Let $P \in \mathcal{C}$ be an object equipped with a filtration $P=P^{0} \supset P^{1} \supset \ldots \supset P^{N}=0$ for $N \geq 0$, with semisimple successive quotients.

Lemma 4.2.1. The following properties are equivalent:

(1) $P$ is a projective object and the filtration is the radical filtration.

(2) For any $i \geq 1$ and any semisimple object $M \in \mathcal{C}$ the map

$$
\operatorname{Hom}\left(P^{i} / P^{i+1}, M\right) \rightarrow \operatorname{Ext}^{1}\left(P / P^{i}, M\right)
$$

coming from the short exact sequence $P^{i} / P^{i+1} \hookrightarrow P / P^{i+1} \rightarrow P / P^{i}$ is bijective.

(3) For any $i \geq 1$ and $L \in \operatorname{IrrC}$ the group $E^{i}(L)=\operatorname{Ext}^{1}\left(P / P^{i}, L\right)$ is a finitely generated $F_{L}$-module, $E^{i}(L)=0$ for all but finitely many $L$, and there exists an isomorphism

$$
P^{i} / P^{i+1} \rightarrow \bigoplus_{L \in \operatorname{IrrC}} E^{i}(L)^{*} \otimes_{F_{L}} L
$$


where $E^{i}(L)^{*}:=\operatorname{Hom}_{F_{L}}\left(E^{i}(L), F_{L}\right)$, such that under the chain of isomorphisms

$$
\begin{aligned}
\operatorname{Ext}^{1}\left(P / P^{i}, P^{i} / P^{i+1}\right) & \rightarrow \operatorname{Ext}^{1}\left(P / P^{i}, \bigoplus_{L} E^{i}(L)^{*} \otimes_{F_{L}} L\right) \\
& =\bigoplus_{L} E^{i}(L)^{*} \otimes_{F_{L}} E^{i}(L) \\
& =\bigoplus_{L} \operatorname{End}_{F_{L}}\left(E^{i}(L)\right)
\end{aligned}
$$

the class of the extension $P^{i} / P^{i+1} \hookrightarrow P / P^{i+1} \rightarrow P / P^{i}$ goes to $\sum_{L} i d_{E^{i}(L)}$.

4.3. Gradings on artinian categories. Let $\mathcal{C}$ be an artinian category (hence an $F$-category by our conventions) with finite dimensional Hom's, and let $\mathcal{M}$ be a mixed category with a degree $d$ Tate twist. Let $v: \mathcal{M} \rightarrow \mathcal{C}$ be an exact faithful functor that sends semisimple objects of $\mathcal{M}$ to semisimple objects of $\mathcal{C}$, and let $\varepsilon: v(M) \mapsto v(M\langle d\rangle), M \in \mathcal{M}$, be a natural isomorphism. We call such a pair $(v, \varepsilon)$ a degrading functor.

For $M, N \in \mathcal{M}$ consider the map

$$
v_{M, N}^{i}: \bigoplus_{n} \operatorname{Ext}_{\mathcal{M}}^{i}(M, N\langle n d\rangle) \rightarrow \operatorname{Ext}_{\mathcal{C}}^{i}(v M, v N)
$$

defined by $v$ and $\varepsilon$. We will be interested in the condition

$(*)_{M, N}^{i}$ : The map $v_{M, N}^{i}$ is bijective.

Definition 4.3.1. (1) A grading on an artinian category $\mathcal{C}$ is defined to be a pair $(\mathcal{M},(v, \varepsilon))$, where $\mathcal{M}$ is a mixed category with a Tate twist and $(v, \varepsilon): \mathcal{M} \rightarrow \mathcal{C}$ is a degrading functor, such that (a) any irreducible object in $\mathcal{C}$ is isomorphic to $v(M)$ for some $M \in \mathcal{M}$, and (b) the condition $(*)_{M, N}^{i}$ holds for all $i$ and all $M, N \in \mathcal{M}$.

(2) An equivalence between two gradings $(\mathcal{M},(v, \varepsilon))$, and $\left(\mathcal{M}^{\prime},\left(v^{\prime}, \varepsilon^{\prime}\right)\right)$ on $\mathcal{C}$ is a pure functor $\phi: \mathcal{M} \rightarrow \mathcal{M}^{\prime}$ compatible with Tate twists together with an isomorphism $v^{\prime} \phi=v$ compatible with $\varepsilon, \varepsilon^{\prime}$.

Remarks. (1) As follows from our definitions, any equivalence $\phi$ as above is actually an equivalence between the categories $\mathcal{M}$ and $\mathcal{M}^{\prime}$.

(2) If $(*)_{M, N}^{i}$ holds for all irreducible $M, N$ and all $i \in \mathbb{Z}$, then $(*)_{M, N}^{i}$ is true for all $M, N, i$.

(3) Any degrading functor $(v, \varepsilon): \mathcal{M} \rightarrow \mathcal{C}$ extends in a unique manner to a degrading functor $\left(v^{1 / d}, \varepsilon^{1 / d}\right): \mathcal{M}^{1 / d} \rightarrow \mathcal{C}$, and $(v, \varepsilon)$ is a grading on $\mathcal{C}$ if and only if $\left(v^{1 / d}, \varepsilon^{1 / d}\right)$ is a grading on $\mathcal{C}$. Therefore we may always pass to the situation with $d=1$.

Examples. (1) Let $A$ be a mixed $F$-algebra, $\mathcal{M}=A$-mof, $\mathcal{C}=A$-Mof the category of all finite dimensional continuous non-graded $A$-modules. Then the forgetthe-grading functor $v: \mathcal{M} \rightarrow \mathcal{C}$ (with the obvious $\varepsilon$ ) is a grading on $\mathcal{C}$. Actually, any grading arises in this way.

(2) The functor $v: \mathcal{H}_{F} \rightarrow$ Vect $_{F}$ that assigns to a Hodge structure its underlying vector space is a degrading functor. To define $\varepsilon$ we must fix a generator in $v(F(1))$. The restriction of $v$ to the subcategory of those Hodge structures that are isomorphic to direct sums of Tate ones is a grading on Vect $F$.

(3) Consider the categories of mixed perverse sheaves in either the Hodge or the $\mathbb{Q}_{l}$ version. The forgetting of mixed structure functor that sends a mixed sheaf to the usual underlying geometric one is a degrading functor. 
Lemma 4.3.2. (1) Let $(v, \varepsilon): \mathcal{M} \rightarrow \mathcal{C}$ be a grading on $\mathcal{C}$. Then:

(a) $v$ sends irreducible objects in $\mathcal{M}$ to irreducible objects in $\mathcal{C}$, and the corresponding map $\operatorname{Irr} \mathcal{M} /\langle\mathbb{Z} d\rangle \rightarrow \operatorname{IrrC}$ is bijective.

(b) The same holds when we replace the word "irreducible" by "indecomposable projective".

(2) Assume that an artinian category $\mathcal{C}$ has enough projective objects and for any $L \in \operatorname{IrrC}$ one has $\operatorname{End} L=F$. Then any degrading functor $(v, \varepsilon): \mathcal{M} \rightarrow \mathcal{C}$ that satisfies the properties (a), (b) above is a grading on $\mathcal{C}$.

Proof. (1) For (a) use $(*)_{M, N}^{0}$ for irreducible $M$ and $N$. For (b), note that an object $P$ in $\mathcal{M}$ is projective if and only if $v(P)$ is projective in $\mathcal{C}$ (use $(*)_{P, N}^{1}$ with $P$ our projective, $N$ any irreducible, and (a)). An argument with $(*)_{P, P}^{0}$ shows that such $P$ is indecomposable if and only if $v(P)$ is. It remains to show that any indecomposable projective object $P_{\mathcal{C}}$ in $\mathcal{C}$ can be lifted to $\mathcal{M}$. To do this consider the radical filtration $P_{\mathcal{C}}=P_{\mathcal{C}}^{0} \supset P_{\mathcal{C}}^{1} \supset \ldots$, and lift $P_{\mathcal{C}} / P_{\mathcal{C}}^{i}$ step by step using 4.2.1 (3) and the above statement (a).

(2) We need to check $(*)_{M, N}^{i}$. It obviously holds when $M$ is projective. For arbitrary $M$, use a projective resolution.

From now on let us assume that $\mathcal{C}$ admits a projective generator $P$, so in particular $\operatorname{IrrC}$ is a finite set. Put $A=(\operatorname{End} P)^{\text {opp }}$. This is a finite dimensional $F$-algebra, and we have a canonical equivalence of categories $\mathcal{C} \rightarrow A$-Mod, $M \mapsto \operatorname{Hom}(P, M)$. Let $\theta$ be a positive grading, $A=\bigoplus A_{\theta}^{i}$ on $A$, such that $A_{\theta}^{0}$ is semisimple (i.e. $A_{\theta}^{>0}=\operatorname{rad} A$ ) or, equivalently, the graded $\operatorname{ring} A_{\theta}$ is a mixed algebra. Set $\tilde{\mathcal{C}}_{\theta}=A_{\theta}$-mof. This is a mixed category with a degree 1 Tate twist; the forget-the-grading functor $\tilde{\mathcal{C}}_{\theta}=A_{\theta}$-mof $\rightarrow A$-Mof $=\mathcal{C}$ is a grading on $\mathcal{C}$.

Lemma 4.3.3. Any grading on $\mathcal{C}$ with a degree 1 Tate twist is equivalent to some $\tilde{\mathcal{C}}_{\theta}$. The gradings $\tilde{\mathcal{C}}_{\theta}, \tilde{\mathcal{C}}_{\theta^{\prime}}$ are equivalent if and only if $\theta^{\prime}=a^{-1} \theta a$ for some $a \in A^{\times}$.

Proof. Let $\mathcal{M} \rightarrow \mathcal{C}$ be a grading with Tate twist of degree 1 . To identify $\mathcal{M}$ with some $\tilde{\mathcal{C}}_{\theta}$ let us choose a lifting $\left(\tilde{P}_{\mathcal{M}}, \alpha\right)$ of $P$ (where $\tilde{P}_{\mathcal{M}}$ is an object of $\mathcal{M}$ and $\alpha: P \rightarrow v\left(\tilde{P}_{\mathcal{M}}\right)$ is an isomorphism) such that the maximal semisimple quotient $T$ of $\tilde{P}_{\mathcal{M}}$ is pure of weight 0 . Such a lifting exists and is uniquely defined up to a change $\alpha \mapsto \alpha a, a \in A^{\times}=(\operatorname{Aut} P)^{\mathrm{opp}}$.

The ring $\tilde{A}=\left(\operatorname{End} v\left(\tilde{P}_{\mathcal{M}}\right)\right)^{\text {opp }}$ is a mixed algebra with respect to its natural grading $\tilde{A}^{i}=\operatorname{Hom}\left(\tilde{P}_{\mathcal{M}}, \tilde{P}_{\mathcal{M}}\langle-i\rangle\right)$. Note that $\tilde{A}$ coincides with the mixed algebra attached to $(\mathcal{M}, T)$ by 4.1.6. The isomorphism $\alpha$ identifies $A$ with $\tilde{A}$, hence defines the grading $\theta=\theta\left(\tilde{P}_{\mathcal{M}}, \alpha\right)$ on $A$. It is clear from Proposition 4.1.6 that the grading $\mathcal{M}$ on $\mathcal{C}$ is equivalent to $\tilde{\mathcal{C}}_{\theta}$. This is the first statement of the lemma. The second one follows since $\theta\left(\tilde{P}_{\mathcal{M}}, \alpha a\right)=a^{-1} \theta\left(\tilde{P}_{\mathcal{M}}, \alpha\right) a$ for any $a \in A^{\times}$.

Remark. In the situation of the lemma it may happen that for two gradings $\theta, \theta^{\prime}$ there exists an automorphism $\phi$ of $A$ such that $\phi(\theta)=\theta^{\prime}$. Such a $\phi$ defines an obvious pure equivalence $\tilde{\mathcal{C}}_{\theta} \cong \tilde{\mathcal{C}}_{\theta^{\prime}}$ which is compatible with projections to $\mathcal{C}$ (i.e. $\phi$ is an equivalence of gradings) iff $\phi$ is an inner automorphism. Note that such $\phi$ always exists if we know that our graded algebras $A_{\theta}, A_{\theta^{\prime}}$ are generated by their components of degree 1 and 0 : Then they are isomorphic to $\operatorname{gr} A=\bigoplus(\operatorname{rad} A)^{i} /(\operatorname{rad} A)^{i+1}$.

4.4. Mixed category $\mathcal{O}: \mathbb{Q}_{l}$-version. Let $X$ be a variety over a finite field $\mathbb{F}_{q}$ equipped with a stratification by smooth affine strata $\left\{X_{\alpha}\right\}$. Set $n_{\alpha}=\operatorname{dim} X_{\alpha}$ and 
denote by $i_{\alpha}: X_{\alpha} \hookrightarrow X$ the embeddings. Let $\mathcal{L}_{\alpha}=i_{\alpha ! *} \mathbb{Q}_{l, X_{\alpha}}\left[n_{\alpha}\right]$ be the intersection cohomology sheaf of $X_{\alpha}$ and $\mathcal{M}_{\alpha}=i_{\alpha !} \mathbb{Q}_{l, X_{\alpha}}\left[n_{\alpha}\right]$ the corresponding "standard" perverse sheaf. Note that $\mathcal{L}_{\alpha}$ is a mixed sheaf in a natural way (actually, a pure one of weight $n_{\alpha}$ ). If we regard $\mathcal{L}_{\alpha}$ as a mixed sheaf, we denote it by $\tilde{\mathcal{L}}_{\alpha}$.

Assume each stratum $X_{\alpha}$ is isomorphic to an affine space $\mathbb{A}_{\mathbb{F}_{q}}^{n_{\alpha}}$. We consider the following condition:

(*): For any $\alpha, \beta$ and $j \in \mathbb{Z}$ the sheaf $H^{j} i_{\beta}^{*} \tilde{\mathcal{L}}_{\alpha}$ on $X_{\beta}$, vanishes if $n_{\alpha}+j$ is odd and is isomorphic to a direct sum of copies of $\mathbb{Q}_{l, X_{\beta}}\left(\left(-n_{\alpha}-j\right) / 2\right)$ if $n_{\alpha}+j$ is even.

To check this condition one may use:

Lemma 4.4.1. Condition $(*)$ is satisfied provided the following two properties hold.

(1) For each $\beta, H^{j} i_{\beta}^{*} \tilde{\mathcal{L}}_{\alpha}$ is a (geometrically) constant sheaf on $X_{\beta}$; this sheaf vanishes whenever $n_{\alpha}+j$ is odd.

(2) The geometric Frobenius acts on $H^{j}\left(X, \tilde{\mathcal{L}}_{\alpha}\right)$ via multiplication by $q^{\left(j+n_{\alpha}\right) / 2}$.

Proof. There is a standard spectral sequence with $E_{2}^{p, q}=\bigoplus_{n_{\beta}=-p} H_{c}^{p+q}\left(X_{\beta}, i_{\beta}^{*} \tilde{\mathcal{L}}_{\alpha}\right)$ that converges to $H_{c}^{p+q}\left(X, \tilde{\mathcal{L}}_{\alpha}\right)\left(H_{c}^{\bullet}\right.$ stands here for the cohomology with compact support). Property (1) yields the parity condition: $E_{2}^{p, q}=0$ whenever $n_{\alpha}+p+q$ is odd. Hence all the differentials $d_{r}, r \geq 2$, vanish, and the spectral sequence degenerates at the $E_{2}$-term. Thus, the group $H_{c}^{j}\left(X_{\beta}, i_{\beta}^{*} \tilde{\mathcal{L}}_{\alpha}\right)$ gets identified in a canonical way with a subquotient of

$$
H_{c}^{j}\left(X, \tilde{\mathcal{L}}_{\alpha}\right) \simeq H^{j}\left(X, \tilde{\mathcal{L}}_{\alpha}\right)^{*}\left(-n_{\alpha}\right)
$$

The property $(2)$ ensures now that the Frobenius acts via multiplication by $q^{\left(j+n_{\alpha}\right) / 2}$ on the groups involved. Note, finally, that the first property yields an isomorphism: $H_{c}^{j}\left(X_{\beta}, i_{\beta}^{*} \tilde{\mathcal{L}}_{\alpha}\right)=H^{0}\left(X_{\beta}, H^{j-2 n_{\beta}} i_{\beta}^{*} \tilde{\mathcal{L}}_{\alpha}\right)\left(-n_{\beta}\right)$, and condition $(*)$ follows.

To apply the lemma in the situation we need, we prove the following result.

Lemma 4.4.2. Assume that for any $\alpha$ there exists a proper surjective morphism $\pi_{\alpha}: Y_{\alpha} \rightarrow \overline{X_{\alpha}}$ such that

(1) $Y_{\alpha}$ is a smooth scheme.

(2) For any $X_{\beta} \subset \overline{X_{\alpha}}$ the projection $\pi_{\alpha}^{-1}\left(X_{\beta}\right) \rightarrow X_{\beta}$ is smooth.

(3) The $\mathbb{Q}_{l}$-cohomology groups of $Y_{\alpha}$ are generated by classes of algebraic cycles defined over $\mathbb{F}_{q}$.

Then the property (2) of Lemma 4.4.1 holds.

Proof. Form the direct image complex $F=\pi_{\alpha *} \mathbb{Q}_{l, Y_{\alpha}}\left[n_{\alpha}\right] \in D_{\text {mixed }}^{b}\left(X, \mathbb{Q}_{l}\right)$. Clearly, $\tilde{\mathcal{L}}_{\alpha}$ is a subquotient of the mixed perverse sheaf ${ }^{p} H^{0} F$. On the other hand, the complex $F$ is a semisimple perverse sheaf (with Frobenius action disregarded), due to the decomposition theorem. Hence the vector space $H^{j}\left(X, \tilde{\mathcal{L}}_{\alpha}\right)$ with the Frobenius action on it is isomorphic to a subquotient of $H^{j}(X, F)=H^{j+n_{\alpha}}\left(Y_{\alpha}, \mathbb{Q}_{l}\right)$. The claim follows.

Corollary 4.4.3. Condition (*) holds for the stratification of the flag variety $G / B$ by Schubert cells.

Proof. Let $U$ be the maximal unipotent subgroup of $G$ so that $U$-orbits are the Schubert cells. Then, clearly, $\tilde{\mathcal{L}}_{\alpha}$ is an $U$-equivariant perverse sheaf. Hence, for all $\beta$ and $j \in \mathbb{Z}$, the sheaf $H^{j} i_{\beta}^{*} \tilde{\mathcal{L}}_{\alpha}$ is $U$-equivariant, hence, a geometrically constant sheaf. 
Now, the first property of Lemma 4.4.1 is proved by Kazhdan-Lusztig [KL80b]. The second property follows from Lemma 4.4.2 applied to the Demazure-Hansen desingularization $Y_{\alpha} \rightarrow \overline{X_{\alpha}}$ of Schubert varieties.

From now on assume that $\left(X,\left\{X_{\alpha}\right\}\right)$ satisfies condition (*) above. Let $\mathcal{P}$ be the Serre subcategory of the category of $\mathbb{Q}_{l}$-perverse sheaves on $X \otimes \overline{\mathbb{F}_{q}}$ generated by the $\mathcal{L}_{\alpha}$, and denote by $\tilde{\mathcal{P}}$ the full subcategory of the category of mixed perverse sheaves on $X$ that consists of those $\tilde{\mathcal{F}}$ for which any $\operatorname{gr}_{j}^{W} \tilde{\mathcal{F}}$ is isomorphic to a direct sum of copies of $\tilde{\mathcal{L}}_{\alpha}\left(\left(n_{\alpha}-j\right) / 2\right)$ with $n_{\alpha}-j$ even. Then $\mathcal{P}$ is an artinian $\mathbb{Q}_{\text {l-category }}$ and $\tilde{\mathcal{P}}$ is a mixed $\mathbb{Q}_{l}$-category with a degree -2 Tate twist. One has $\operatorname{Irr} \mathcal{P}=\left\{\mathcal{L}_{\alpha}\right\}$, $\operatorname{Irr} \tilde{\mathcal{P}}=\left\{\tilde{\mathcal{L}}_{\alpha}(n)\right\}$. We have the forgetting of mixed structure functor $v: \tilde{\mathcal{P}} \rightarrow \mathcal{P}$. Choose a generator $\varepsilon$ of $\mathbb{Q}_{l}(1)$. Then $(v, \varepsilon)$ is a degrading functor. The following theorem is the main result of this section. Its proof will occupy the rest of it.

Theorem 4.4.4. $(1)(v, \varepsilon): \tilde{\mathcal{P}} \rightarrow \mathcal{P}$ is grading on $\mathcal{P}$.

(2) $\tilde{\mathcal{P}}$ is a Koszul category.

Let us start the proof with

Lemma 4.4.5. For a constructible complex $\mathcal{G} \in D\left(X \otimes \overline{\mathbb{F}_{q}}, \mathbb{Q}_{l}\right)$ the following conditions are equivalent:

(1) ${ }^{p} H^{j}(\mathcal{G}) \in \mathcal{P}$ for all $j \in \mathbb{Z}$.

(2) The $H^{j} i_{\beta}^{*}(\mathcal{G})$ are constant sheaves on $X_{\beta}$ for all $\beta$ and $j \in \mathbb{Z}$.

Proof. The sheaves that satisfy (1) or (2) form the full triangulated subcategories $D_{1}, D_{2}$ of $D\left(X \otimes \overline{\mathbb{F}_{q}}, \mathbb{Q}_{l}\right)$. The category $D_{1}$ is generated by the $\left\{\mathcal{L}_{\alpha}\right\}$, the category $D_{2}$ by the $\left\{\mathcal{M}_{\alpha}\right\}$. By $(*)$ we have $\mathcal{L}_{\alpha} \in D_{2}$, and therefore $D_{1} \subset D_{2}$. To prove the converse inclusion it suffices to show that all $\mathcal{M}_{\alpha}$ lie in $\mathcal{P}$. We do it using induction on the number of strata. Let $X_{\omega}$ be an open stratum. By induction we know that any perverse sheaf $\mathcal{G}$ supported on $X-X_{\omega}$ that satisfies (2) automatically lies in $\mathcal{P}$; in particular this holds for $\mathcal{M}_{\alpha}$ whenever $\alpha \neq \omega$. Consider the canonical surjection $\mathcal{M}_{\omega} \rightarrow \mathcal{L}_{\omega}$. Let $\mathcal{K}_{\omega}$ be its kernel. Then $\mathcal{K}_{\omega}$ is supported on $X-X_{\omega}$, and $H^{j} i_{\beta}^{*} \mathcal{K}_{\omega}=H^{j-1} i_{\beta}^{*} \mathcal{L}_{\omega}$ for any $\beta \neq \omega$. From $(*)$ we deduce that $\mathcal{K}_{\omega} \in \mathcal{P}$, hence $\mathcal{M}_{\omega} \in \mathcal{P}$.

Remark. Actually the above arguments prove the following general statement. Let $X$ be any variety stratified by smooth strata $X_{\alpha}$, put $n_{\alpha}=\operatorname{dim} X_{\alpha}$, and assume that for each $X_{\alpha}$ we picked a Serre subcategory $\mathcal{C}_{\alpha}$ of the category of lisse sheaves on $X_{\alpha}$. Assume that for any $\alpha, \beta$ and $\mathcal{F} \in \operatorname{Irr} \mathcal{C}_{\alpha}$ the sheaves $H^{j} i_{\beta}^{*} i_{\alpha ! *} \mathcal{F}, j \in \mathbb{Z}$, lie in $\mathcal{C}_{\beta}$. Then the full triangulated subcategory of $D\left(X, \mathbb{Q}_{l}\right)$ generated by the perverse sheaves $i_{\alpha ! *}(\mathcal{F}), \mathcal{F} \in \operatorname{IrrC}_{\alpha}$, consists precisely of those complexes $\mathcal{G}$ which satisfy the condition $H^{j}\left(i_{\beta}^{*} \mathcal{G}\right) \in \mathcal{C}_{\beta}$ for all $\beta$ and $j \in \mathbb{Z}$.

Lemma 4.4.6. (1) The category $\mathcal{P}$ has enough projectives.

(2) The obvious functor $D^{b}(\mathcal{P}) \rightarrow D\left(X \otimes \overline{\mathbb{F}_{q}}, \mathbb{Q}_{l}\right)$ is a fully faithful embedding.

Proof. The parallel statement in the situation when $X$ is a complex variety (with condition $(*)$ dropped) was proven in 3.3. The reader may easily check that the same proof works in our present situation, provided we know that the perverse sheaves $\mathcal{M}_{\alpha}$ lies in $\mathcal{P}$. And this was the preceding lemma. 
If $\mathcal{F}, \mathcal{G}$ are perverse sheaves on $X \otimes \overline{\mathbb{F}_{q}}$, we set

$$
\operatorname{Ext}^{i}(\mathcal{F}, \mathcal{G}):=\operatorname{Hom}_{D\left(X \otimes \overline{\mathbb{F}}_{q}, \mathbb{Q}_{l}\right)}(\mathcal{F}, \mathcal{G}[i])
$$

This group also coincides with the Yoneda Ext in the category of all perverse sheaves on $X$. The above lemma claims, in particular, that for any $\mathcal{F}, \mathcal{G} \in \mathcal{P}$ the obvious map $\operatorname{Ext}_{\mathcal{P}}^{i}(\mathcal{F}, \mathcal{G}) \rightarrow \operatorname{Ext}^{i}(\mathcal{F}, \mathcal{G})$ is an isomorphism. For a pair of irreducibles $\mathcal{L}_{\alpha}, \mathcal{L}_{\beta}$ consider the $\mathbb{Q}_{l}$-vector space $\operatorname{Ext}^{1}\left(\mathcal{L}_{\alpha}, \mathcal{L}_{\beta}\right)$. The mixed structure $\tilde{\mathcal{L}_{\alpha}}, \tilde{\mathcal{L}_{\beta}}$ defines a Frobenius action on it.

Lemma 4.4.7. If $n_{\alpha}-n_{\beta}$ is even, then $\operatorname{Ext}^{1}\left(\mathcal{L}_{\alpha}, \mathcal{L}_{\beta}\right)=0$. If $n_{\alpha}-n_{\beta}$ is odd, then the Frobenius acts on $\operatorname{Ext}^{1}\left(\mathcal{L}_{\alpha}, \mathcal{L}_{\beta}\right)$ as multiplication by $q^{\left(n_{\beta}-n_{\alpha}+1\right) / 2}$.

Proof. We have a canonical isomorphism

$$
\operatorname{Ext}^{1}\left(\mathcal{L}_{\alpha}, \mathcal{L}_{\beta}\right)=\operatorname{Ext}^{1}\left(D \mathcal{L}_{\beta}, D \mathcal{L}_{\alpha}\right)=\operatorname{Ext}^{1}\left(\mathcal{L}_{\beta}, \mathcal{L}_{\alpha}\right)\left(n_{\alpha}-n_{\beta}\right)
$$

the latter since $D \mathcal{L}_{\alpha}=\mathcal{L}\left(n_{\alpha}\right)$. Therefore we may assume that $\overline{X_{\alpha}}-X_{\alpha} \not \supset X_{\beta}$ (otherwise interchange $\alpha$ and $\beta$ in the above formula). The short exact sequence $\mathcal{K}_{\alpha} \hookrightarrow \mathcal{M}_{\alpha} \rightarrow \mathcal{L}_{\alpha}$ provides the exact sequence $\operatorname{Hom}\left(\mathcal{K}_{\alpha}, \mathcal{L}_{\beta}\right) \rightarrow \operatorname{Ext}^{1}\left(\mathcal{L}_{\alpha}, \mathcal{L}_{\beta}\right) \rightarrow$ $\operatorname{Ext}^{1}\left(\mathcal{M}_{\alpha}, \mathcal{L}_{\beta}\right)$. Since our condition implies that $\operatorname{Hom}\left(\mathcal{K}_{\alpha}, \mathcal{L}_{\beta}\right)=0$, we see that

$$
\operatorname{Ext}^{1}\left(\mathcal{L}_{\alpha}, \mathcal{L}_{\beta}\right) \hookrightarrow \operatorname{Ext}^{1}\left(\mathcal{M}_{\alpha}, \mathcal{L}_{\beta}\right)=H^{1}\left(X_{\alpha} \otimes \overline{\mathbb{F}_{q}}, i_{\alpha}^{*}\left(\mathcal{L}_{\beta}\right)\left[-n_{\alpha}\right]\right)
$$

so it suffices to check the statement of our lemma for this larger group. Since the $X_{\alpha}$ are supposed to be just affine linear spaces, then this coincides with $H^{0}\left(X_{\alpha} \otimes \overline{\mathbb{F}_{q}}, H^{1-n_{\alpha}} i_{\alpha}^{*} \mathcal{L}_{\beta}\right)$, and by $(*)$ we are done.

Lemma 4.4.8. Let $P$ be an indecomposable projective in $\mathcal{P}$. Then:

(1) $P$ can be lifted to $\tilde{\mathcal{P}}$, i.e. there exists $\tilde{P} \in \tilde{\mathcal{P}}$ together with an isomorphism $v(\tilde{P}) \rightarrow P$.

(2) Any such $\tilde{P}$ is a projective object of $\tilde{\mathcal{P}}$.

Proof. We need use the following general fact. Let $\tilde{\mathcal{F}}_{1}, \tilde{\mathcal{F}}_{2}$ be mixed perverse sheaves. Then one has a canonical short exact sequence

$$
\operatorname{Hom}\left(\mathcal{F}_{1}, \mathcal{F}_{2}\right)_{F r} \hookrightarrow \operatorname{Ext}^{1}\left(\tilde{\mathcal{F}}_{1}, \tilde{\mathcal{F}}_{2}\right) \rightarrow \operatorname{Ext}^{1}\left(\mathcal{F}_{1}, \mathcal{F}_{2}\right)^{F r}
$$

where $\mathcal{F}_{i}=v\left(\tilde{\mathcal{F}}_{i}\right)$ are the underlying usual perverse sheaves, and the middle Ext ${ }^{1}$ is the group of extensions in the category of all mixed sheaves (not necessarily $\operatorname{gr}_{\bullet}^{W}$-semisimple ones).

We now prove $(1)$. Let $P=P^{0} \supset P^{1} \supset \ldots$ be the radical filtration on $P$. We find a lifting $\tilde{P}$ such that $P^{i}=v\left(W_{w-i}(\tilde{P})\right)$ for some $w \in \mathbb{Z}$. To do this we inductively define liftings $\widehat{P / P^{i}}$ of $P / P^{i}$ such that $\widehat{P / P^{i+1}}$ is an extension of $\widetilde{P / P^{i}}$ by a pure sheaf $\widetilde{P^{i} / P^{i+1}}$ of weight $w-i$. To start the induction, note that $P / P^{1}$ is irreducible, hence can be lifted to some $\widehat{P / P^{1}}$. Set $w=w\left(\widehat{P / P^{1}}\right)$. Now assume we already have $\widetilde{P / P^{i}}$. Consider the isomorphism

$$
P^{i} / P^{i+1} \rightarrow \bigoplus_{\alpha} \operatorname{Ext}^{1}\left(P / P^{i}, \mathcal{L}_{\alpha}\right)^{*} \otimes_{\mathbb{Q}_{l}} \mathcal{L}_{\alpha}
$$

from 4.2.1 (3). The mixed structure $\widetilde{P / P^{i}}$ and the standard mixed structure $\tilde{\mathcal{L}_{\alpha}}$ on $\mathcal{L}_{\alpha}$ define a Frobenius action on $\operatorname{Ext}^{1}\left(P / P^{i}, \mathcal{L}_{\alpha}\right)$. Since our filtration is the radical filtration, the restriction map $\operatorname{Ext}^{1}\left(P / P^{i}, \mathcal{L}_{\alpha}\right) \rightarrow \operatorname{Ext}^{1}\left(P^{i-1} / P^{i}, \mathcal{L}_{\alpha}\right)$ is injective. By our induction hypothesis, $\widehat{P^{i-1} / P^{i}}=W_{w-i+1}\left(\widehat{P / P^{i}}\right)$ is a pure 
(hence semisimple) object of $\tilde{\mathcal{P}}$ of weight $w-i+1$. By 4.4.7 the Frobenius acts on $\operatorname{Ext}^{1}\left(P^{i-1} / P^{i}, \mathcal{L}_{\alpha}\right)$ as multiplication by $q^{\left(n_{\alpha}-w+i\right) / 2}$ (and it vanishes if $n_{\alpha}-w+i$ is an odd integer). Therefore the same is true for $\operatorname{Ext}^{1}\left(P / P^{i}, \mathcal{L}_{\alpha}\right)$, and we may consider $\bigoplus_{\alpha} \operatorname{Ext}^{1}\left(P / P^{i}, \mathcal{L}_{\alpha}\right)^{*} \otimes \mathcal{L}_{\alpha}$ as a pure object of $\tilde{\mathcal{P}}$ of weight $w-i$. This defines the pure structure $\widehat{P^{i} / P^{i+1}}$ on $P^{i} / P^{i+1}$. The class of the extension $P^{i} / P^{i+1} \hookrightarrow P / P^{i+1} \rightarrow P / P^{i}$ is invariant with respect to the Frobenius-action on $\operatorname{Ext}^{1}\left(P / P^{i}, P^{i} / P^{i+1}\right)$ defined by the mixed structures $\widetilde{P / P^{i}}$ and $\widetilde{P^{i} / P^{i+1}}$ (see 4.2.1 (3)); hence it comes from an extension $\widehat{P^{i} / P^{i+1}} \hookrightarrow \widehat{P / P^{i+1}} \rightarrow \widehat{P / P^{i}}$. Clearly $\widetilde{P / P^{i+1}} \in \tilde{\mathcal{P}}$, and we are done.

Next we prove $(2)$. We need to show that $\operatorname{Ext}_{\tilde{\mathcal{P}}}^{1}(\tilde{P}, \tilde{\mathcal{L}})=0$ for any $\tilde{\mathcal{L}} \in \operatorname{Irr} \tilde{\mathcal{P}}$.

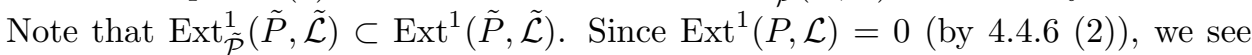
that $\operatorname{Ext}_{\tilde{\mathcal{P}}}^{1}(\tilde{P}, \tilde{\mathcal{L}}) \subset \operatorname{Hom}(P, \mathcal{L})_{F r}=\operatorname{Hom}\left(P / P^{1}, \mathcal{L}\right)_{F r}$. This group is non-zero if and only if $\widetilde{P / P^{1}} \cong \tilde{\mathcal{L}}$. In this case $\operatorname{Ext}_{\tilde{\mathcal{P}}}^{1}(\tilde{P}, \tilde{\mathcal{L}})=0$ (the corresponding extensions are not $\mathrm{gr}^{W}$-semisimple), and we are done.

Proof of 4.4.4. (1) We may use the criterion 4.3 .2 (2) by 4.4.6 (1). The condition 4.3.2 (a) is obvious, while 4.3.2 (b) follows from 4.4.8.

(2) We have to show that for any $\tilde{\mathcal{L}}_{1}, \tilde{\mathcal{L}}_{2} \in \operatorname{Irr} \tilde{\mathcal{P}}$ of weights $w_{1}, w_{2}$ the extension $\operatorname{Ext}_{\tilde{\mathcal{P}}}^{i}\left(\tilde{\mathcal{L}}_{1}, \tilde{\mathcal{L}}_{2}\right)$ vanishes unless $i=w_{1}-w_{2}$. For this it is sufficient, by 3.4 .2 , to establish an injection $\operatorname{Ext}_{\tilde{\mathcal{P}}}^{i}\left(\tilde{\mathcal{L}}_{1}, \tilde{\mathcal{L}}_{2}\right) \hookrightarrow \operatorname{Ext}^{i}\left(\mathcal{L}_{1}, \mathcal{L}_{2}\right)^{F r}$. This we deduce from the following commutative diagram:

$$
\begin{array}{cccc}
\operatorname{Ext}_{\tilde{\mathcal{P}}}^{i}\left(\tilde{\mathcal{L}}_{1}, \tilde{\mathcal{L}}_{2}\right) & \hookrightarrow & \operatorname{Ext}_{\mathcal{P}}^{i}\left(\mathcal{L}_{1}, \mathcal{L}_{2}\right) \\
\downarrow & & \| \\
\operatorname{Ext}^{i}\left(\tilde{\mathcal{L}}_{1}, \tilde{\mathcal{L}}_{2}\right) & \rightarrow \operatorname{Ext}^{i}\left(\mathcal{L}_{1}, \mathcal{L}_{2}\right) & & \\
& & & \operatorname{Ext}^{i}\left(\mathcal{L}_{1}, \mathcal{L}_{2}\right) .
\end{array}
$$

Here the upper horizontal is an injection by $4.4 .4(1)$, the right vertical is an isomorphism by 4.4.6(2), the left vertical comes from the fact that the upper left corner is a universal $\partial$-functor, and the left map in the lower horizontal is the end of the short exact sequence from the proof of Lemma 4.4.8.

4.5. Mixed category $\mathcal{O}$ : Hodge version. Let $X$ be an algebraic variety over $\mathbb{C}$. Fix a subfield $F \subset \mathbb{R}$. Denote by $\mathcal{P}$ the category of perverse $F$-sheaves on $X$ and by $\mathcal{P}_{\mathcal{H}}$ the category of Hodge $F$-modules (i. e. mixed Hodge sheaves) on $X$. We have the functor $v: \mathcal{P}_{\mathcal{H}} \rightarrow \mathcal{P}$ of forgetting Hodge structure. If we choose a generator $\varepsilon$ of the 1 -dimensional vector space $F(1)=F \cdot 2 \pi \sqrt{-1}$, then $(v, \varepsilon)$ is a degrading functor (see 4.3).

Let $\mathcal{C} \subset \mathcal{P}$ be a Serre subcategory. We consider the following problem: Find a mixed subcategory $\tilde{\mathcal{C}} \subset \mathcal{P}_{\mathcal{H}}$, closed with respect to Tate twist, such that $\tilde{\mathcal{C}} \rightarrow \mathcal{C}$ is a grading on $\mathcal{C}$. This problem (for given $\mathcal{C}$ ) seems to be quite difficult. For example, we have no idea if $\mathcal{C}$ exists in the generality of 4.4 or 3.4 (i.e. in case when $\mathcal{C}$ consists of sheaves smooth along a stratification by affine planes). However, in the very particular case when $X=G / B$ and $\mathcal{C}$ coincides with the category $\mathcal{O}$ of perverse sheaves lisse along the Schubert stratification $\left\{X_{\alpha}\right\}$, the category $\tilde{\mathcal{C}}$ does exist. We will construct it below.

Let $X=G / B,\left\{X_{\alpha}\right\}, \mathcal{O}$ be as above and let $\mathcal{O}_{\mathcal{H}}:=v^{-1} \mathcal{O}$ be the category of Hodge modules lisse along $\left\{X_{\alpha}\right\}$. Note that each $\mathcal{L}_{\alpha}$ carries a canonical Hodge structure (of weight $n_{\alpha}$ ). Denote this irreducible object of $\mathcal{O}_{\mathcal{H}}$ by $\tilde{\mathcal{L}}_{\alpha}$. 
Lemma 4.5.1. Any Hodge structure on $\mathcal{L}_{\alpha}$ is isomorphic to the twist $\tilde{\mathcal{L}}_{\alpha}(j)$ for some $j \in \mathbb{Z}$.

Proof. Let $\tilde{\mathcal{L}}_{\alpha}^{1}$ be a Hodge structure on $\mathcal{L}_{\alpha}$. Let us consider the canonical isomorphism $\operatorname{Hom}\left(\mathcal{L}_{\alpha}, \mathcal{L}_{\alpha}\right) \otimes \mathcal{L}_{\alpha} \cong \mathcal{L}_{\alpha}$ (note that $\left.\operatorname{Hom}\left(\mathcal{L}_{\alpha}, \mathcal{L}_{\alpha}\right)=F\right)$. If we put on $F=\operatorname{Hom}\left(\mathcal{L}_{\alpha}, \mathcal{L}_{\alpha}\right)$ the Hodge structure $\tilde{F}$ defined by $\tilde{\mathcal{L}}_{\alpha}$ and $\tilde{\mathcal{L}}_{\alpha}^{1}$, then this will give the isomorphism of Hodge sheaves $\tilde{F} \otimes \tilde{\mathcal{L}}_{\alpha} \cong \tilde{\mathcal{L}}_{\alpha}^{1}$. Since $\operatorname{dim} F=1$, the only possible $\tilde{F}$ is $F(j)$.

Recall some general facts about Hodge sheaves (see [Sai90]). If $\tilde{\mathcal{F}}$ is a Hodge sheaf on $X$ and $\mathcal{F}$ is the underlying perverse sheaf, then the groups $H^{j}(X, \mathcal{F})$ carry a natural Hodge structure. We denote it by $H^{j}(X, \tilde{\mathcal{F}}) \in \mathcal{H}_{F}$. More generally, for $\tilde{\mathcal{F}}_{1}, \tilde{\mathcal{F}}_{2}$ the groups $\operatorname{Ext}^{j}\left(\mathcal{F}_{1}, \mathcal{F}_{2}\right)$ carry a natural Hodge structure. We denote it by $\operatorname{Ext}^{j}\left(\tilde{\mathcal{F}}_{1}, \tilde{\mathcal{F}}_{2}\right) \in \mathcal{H}_{F}$. We have a canonical spectral sequence converging to $\operatorname{Ext}_{\mathcal{H}}^{\bullet}\left(\tilde{\mathcal{F}}_{1}, \tilde{\mathcal{F}}_{2}\right)$ (:= Ext groups in the category of Hodge sheaves) with first term $E_{1}^{p, q}=H_{\mathcal{H}}^{2 p+q}\left(\operatorname{Ext}^{-p}\left(\tilde{\mathcal{F}}_{1}, \tilde{\mathcal{F}}_{2}\right)\right)$. Here $H_{\mathcal{H}}^{j}$ is the absolute Hodge cohomology functor: For a Hodge structure $M \in \mathcal{H}_{F}$ we have $H_{\mathcal{H}}^{j}(M):=\operatorname{Ext}_{\mathcal{H}_{F}}^{j}(F(0), M)$. Since $H_{\mathcal{H}}^{j}=0$ for $j \neq 0,1$ this spectral sequence degenerates at $E_{2}$. In particular, we have a short exact sequence

$$
H_{\mathcal{H}}^{1} \operatorname{Hom}\left(\tilde{\mathcal{F}}_{1}, \tilde{\mathcal{F}}_{2}\right) \hookrightarrow \operatorname{Ext}_{\mathcal{H}}^{1}\left(\tilde{\mathcal{F}}_{1}, \tilde{\mathcal{F}}_{2}\right) \rightarrow H_{\mathcal{H}}^{0} \operatorname{Ext}^{1}\left(\tilde{\mathcal{F}}_{1}, \tilde{\mathcal{F}}_{2}\right)
$$

Lemma 4.5.2. (1) For any $\alpha, \beta$ and $j \in \mathbb{Z}$ the sheaf $H^{j} i_{\beta}^{*} \tilde{\mathcal{L}}_{\alpha}$ on $X_{\beta}$ vanishes if $j+n_{\alpha}$ is odd. It is isomorphic to a direct sum of some copies of $F\left(\left(-j-n_{\alpha}\right) / 2\right)$ if $j+n_{\alpha}$ is even.

(2) The cohomology group $H^{j}\left(X, \tilde{\mathcal{L}}_{\alpha}\right)$ vanishes if $j+n_{\alpha}$ is odd. It is isomorphic to a direct sum of some copies of $F\left(\left(-j-n_{\alpha}\right) / 2\right)$ if $j+n_{\alpha}$ is even.

(3) The group $\operatorname{Ext}^{j}\left(\tilde{\mathcal{L}}_{\alpha}, \tilde{\mathcal{L}}_{\beta}\right)$ vanishes if $j-n_{\alpha}+n_{\beta}$ is odd. It is isomorphic to a direct sum of some copies of $F\left(\left(-j-n_{\alpha}+n_{\beta}\right) / 2\right)$ if $j-n_{\alpha}+n_{\beta}$ is even.

Proof. (1) (cf. 4.4.2). Use the Demazure-Hansen desingularization $Y_{\alpha}$ of $\overline{X_{\alpha}}$ and the decomposition theorem.

(2) follows from (1), since the terms of the spectral sequence for computing $H^{j}\left(X, \tilde{\mathcal{L}}_{\alpha}\right)$ via the stratification $\left\{X_{\alpha}\right\}$ have the properties listed in (2) and any extension between Tate (or any Hodge) structures of the same weight is trivial. Alternatively, use the decomposition theorem directly to identify $H^{\bullet}\left(X, \mathcal{L}_{\alpha}\right)$ with pieces of the cohomology of $Y_{\alpha}$.

(3) To compute

$$
\operatorname{Ext}^{\bullet}\left(\tilde{\mathcal{L}}_{\alpha}, \tilde{\mathcal{L}}_{\beta}\right)=H^{\bullet}\left(X, D\left(\tilde{\mathcal{L}}_{\alpha} \otimes D \tilde{\mathcal{L}}_{\beta}\right)\right)=H^{\bullet}\left(X, \tilde{\mathcal{L}}_{\alpha} \otimes \tilde{\mathcal{L}}_{\beta}\right)^{*}\left(-n_{\beta}\right)
$$

one may use, as in (2), the spectral sequence for the stratification $\left\{X_{\alpha}\right\}$. Again, by (1), its terms satisfy the properties listed in (3); hence this is true for the Ext ${ }^{\bullet}$-groups themselves. Alternativly, by 3.4.2 the canonical map $\operatorname{Ext}^{j}\left(\mathcal{L}_{\alpha}, \mathcal{L}_{\beta}\right) \rightarrow$ $\operatorname{Hom}\left(H^{\bullet}\left(X, \mathcal{L}_{\alpha}\right), H^{\bullet+j}\left(X, \mathcal{L}_{\beta}\right)\right)$ is injective. Since it is obviously compatible with Hodge structures, we are done by $(2)$.

Lemma 4.5.3. Let $P \rightarrow \mathcal{L}_{\alpha}$ be a projective cover in $\mathcal{O}$ and $P=P^{0} \supset P^{1} \supset \ldots$ the radical filtration of $P$.

(1) There exists a Hodge structure $\tilde{P}$ on $P$ such that $\tilde{P} \rightarrow \tilde{\mathcal{L}}_{\alpha}$ is a morphism of Hodge sheaves. 
(2) For any such $\tilde{P}$ one has $P^{i}=v\left(W_{n_{\alpha}-i} \tilde{P}\right)$, and $\widetilde{P^{i} / P^{i+1}}:=\operatorname{gr}_{n_{\alpha}-i}^{W}(\tilde{P})$ is a direct sum of some objects of the form $\tilde{\mathcal{L}}_{\beta}(j)$ (of weight $n_{\alpha}-i$ ).

Proof. Let us start with (2). By induction on $i$ we may assume that $P^{j}$ is a Hodge subsheaf of $\tilde{P}$ and $P^{j-1} / P^{j}$ is a direct sum of Tate twists of some $\tilde{\mathcal{L}}_{\beta}$ of weight $n_{\alpha}-j+1$ for any $j \leq i$. We need to show that $P^{i+1}$ is a Hodge subsheaf and $P^{i} / P^{i+1}$ is a sum of some objects of the form $\tilde{\mathcal{L}}_{\beta}(j)$ of weight $n_{\alpha}-i$. First consider the canonical morphism $\gamma: \tilde{P} \rightarrow \bigoplus_{\beta} \operatorname{Hom}\left(\tilde{P}^{i}, \tilde{\mathcal{L}}_{\beta}\right)^{*} \otimes \tilde{\mathcal{L}}_{\beta}$ coming from the obvious maps $\operatorname{Hom}\left(\tilde{P}^{i}, \tilde{\mathcal{L}}_{\beta}\right) \otimes \tilde{P}^{i} \rightarrow \tilde{\mathcal{L}}_{\beta}$. Its kernel is $P^{i+1}$; hence this is a Hodge subsheaf of $\tilde{P}^{i}$. The canonical isomorhism 4.2.1(3)

$$
\tilde{P}^{i} / \tilde{P}^{i+1} \rightarrow \bigoplus_{\beta} \operatorname{Ext}^{1}\left(\tilde{P} / \tilde{P}^{i}, \tilde{\mathcal{L}_{\beta}}\right)^{*} \otimes_{F} \tilde{\mathcal{L}}_{\beta}
$$

is obviously compatible with Hodge structures. Since our filtration is the radical one, the restriction map $\operatorname{Ext}^{1}\left(P / P^{i}, \mathcal{L}_{\beta}\right) \rightarrow \operatorname{Ext}^{1}\left(P^{i-1} / P^{i}, \mathcal{L}_{\beta}\right)$ is injective. Since, by induction, $\tilde{P}^{i-1} / \tilde{P}^{i}$ is a direct sum of Tate twists of $\tilde{\mathcal{L}}_{\gamma}$ 's of weight $n_{\alpha}-i+1$, we are done by part (3) of the preceding lemma.

To prove existence of $\tilde{P}$ we again use induction over $i$. Namely, we build the $\widetilde{P / P_{i}}$ successively. The procedure is identical with the one used in the proof of 4.4 .8 (1). We should only replace the words "Frobenius action" by "Hodge structure" and use the short exact sequence preceding Lemma 4.5.2 instead of the one from the beginning of the proof of 4.4.8.

Now consider the skyscraper sheaf $\mathcal{L}_{e}$ supported at the point orbit. Let $\tilde{P}_{e} \rightarrow \tilde{\mathcal{L}}_{e}$ be a morphism of Hodge sheaves such that $P_{e} \rightarrow \mathcal{L}_{e}$ is a projective covering of $\mathcal{L}_{e}$. Define $\tilde{\mathcal{O}}_{\tilde{P}_{e}}$ to be the smallest full abelian subcategory of $\mathcal{O}_{\mathcal{H}}$ containing all $\tilde{P}_{e}(j)$. It consists of all subquotients of direct sums of objects of the form $\tilde{P}_{e}(j)$.

Theorem 4.5.4. (1) $\tilde{\mathcal{O}}_{\tilde{P}_{e}} \rightarrow \mathcal{O}$ is a grading on $\mathcal{O}$.

(2) $\tilde{\mathcal{O}}_{\tilde{P}_{e}}$ is a Koszul category.

Proof. First note that (2) follows from (1) . Namely, by (1) and 4.5.1 we know that $\operatorname{Irr} \tilde{\mathcal{O}}_{\tilde{P}_{e}}=\left\{\tilde{\mathcal{L}}_{\alpha}(n)\right\}$, where $w\left(\tilde{\mathcal{L}}_{\alpha}(n)\right)=n_{\alpha}-2 n$; therefore Koszulity means that $\operatorname{Ext}_{\tilde{\mathcal{O}}_{\tilde{P}_{e}}}^{i}\left(\tilde{\mathcal{L}}_{\alpha}(a), \tilde{\mathcal{L}}_{\beta}(b)\right)$ vanishes if $i \neq n_{\alpha}-n_{\beta}-2 a+2 b$. By (1) the mapping $\operatorname{Ext}_{\tilde{\mathcal{O}}_{\tilde{P}}}^{i}\left(\left(\tilde{\mathcal{L}}_{\alpha}(a), \tilde{\mathcal{L}}_{\beta}(b)\right) \rightarrow \operatorname{Ext}^{i}\left(\mathcal{L}_{\alpha}, \mathcal{L}_{\beta}\right)\right.$ is injective. Since it factors through $\operatorname{Ext}_{\mathcal{H}}^{i}\left(\tilde{\mathcal{L}}_{\alpha}(a), \tilde{\mathcal{L}}_{\beta}(b)\right)$, its image lies in $H_{\mathcal{H}}^{0}\left(\operatorname{Ext}^{i}\left(\tilde{\mathcal{L}}_{\alpha}(a), \tilde{\mathcal{L}}_{\beta}(b)\right)\right.$, and we are done by 4.5.2(3).

To prove (1) we will use the following lemma. Let $\tilde{C}^{\bullet}$ be the maximal $W$ invariant quotient algebra of $\operatorname{Sym}_{1}(H, F)$. Here $H$ is a Cartan subgroup of $G$, and we consider $H_{1}(H, F)$ as a Hodge structure. Clearly $H_{1}(H, F)=\Gamma_{H} \otimes F(1)$, where $\Gamma_{H}$ is the lattice of 1-parameter subgroups in $H$. Therefore $\tilde{\mathcal{C}}^{\bullet}$ is a commutative algebra in $\mathcal{H}_{F}$, graded in an obvious manner. Consider the corresponding graded $F$-algebra $C^{\bullet}=v\left(\tilde{C}^{\bullet}\right)$. According to [Soe90] we have a canonical isomorphism $C^{\bullet} \cong \operatorname{End} P_{e}$.

Lemma 4.5.5. This isomorphism is compatible with Hodge structures, i.e. $\tilde{C} \cong$ End $\tilde{P}_{e}$.

Proof. Proof postponed to the next subsection. 
We say that $\tilde{\mathcal{F}} \in \tilde{\mathcal{O}}_{\tilde{P}_{e}}$ is a special object if it is isomorphic to a quotient of a direct sum of copies of $\tilde{P}_{e}(n)$ 's.

Step 1. Let $\tilde{\mathcal{F}}$ and $\tilde{\mathcal{G}}$ be objects of $\tilde{\mathcal{O}}_{\tilde{P}_{e}}$, and suppose $\tilde{\mathcal{F}}$ is special. Then $\operatorname{Hom}(\tilde{\mathcal{F}}, \tilde{\mathcal{G}})$ is a direct sum of Tate structures $F(i)$. This follows immediately from projectivity of $P_{e}$ and the preceding lemma, since $\operatorname{our} \operatorname{Hom}(\tilde{\mathcal{F}}, \tilde{\mathcal{G}})$ is a subquotient of a sum of Hodge structures isomorphic to $\left(\operatorname{End} \tilde{P}_{e}\right)(i)$.

Step 2. For any $\alpha$ there exists a special object $\tilde{I}_{\alpha}$ such that $I_{\alpha}:=v\left(\tilde{I}_{\alpha}\right)$ is an injective envelope $\left(:=\right.$ an indecomposable injective object) of $\mathcal{L}_{\alpha}$. Indeed, according to [Soe90] there exists a graded $C^{\bullet}$-module $V_{\alpha}^{\bullet}$ such that $I_{\alpha}:=V_{\alpha} \otimes_{C} P_{e}$ is an injective envelope of $\mathcal{L}_{\alpha}$ (here $V_{\alpha}$ is $V_{\alpha}^{\bullet}$ with its grading forgotten). Now consider the Hodge structure $\tilde{V}_{\alpha}:=\bigoplus V_{\alpha}^{i}(i)$, and set $\tilde{I}_{\alpha}:=\tilde{V}_{\alpha} \otimes_{\tilde{C}} \tilde{P}_{e}$. Note that $\operatorname{dim} \operatorname{Hom}\left(\mathcal{L}_{\alpha}, I_{\alpha}\right)=1$; therefore we may assume, as in the proof of 4.5.1, after possible twisting of $I_{\alpha}$, that $\tilde{\mathcal{L}}_{\alpha} \subset \tilde{I}_{\alpha}$.

Step 3 . The category $\tilde{\mathcal{O}}_{\tilde{P}_{e}}$ consists precisely of those sheaves $\tilde{\mathcal{F}} \in \mathcal{O}_{\mathcal{H}}$ such that $\operatorname{Hom}\left(\tilde{\mathcal{F}}, \tilde{I}_{\alpha}\right)$ is a direct sum of Tate structures for any $\alpha$. Indeed, if $\tilde{\mathcal{F}}$ is a special object of $\tilde{\mathcal{O}}_{\tilde{P}_{e}}$, then we may embed it into a special one and use injectivity of $I_{\alpha}$ to get our claim. Conversely, assume that $\tilde{\mathcal{F}}$ has the property that all $\operatorname{Hom}\left(\tilde{\mathcal{F}}, \tilde{I}_{\alpha}\right)$ are direct sums of Tate structures. The canonical embedding $\tilde{\mathcal{F}} \hookrightarrow \bigoplus_{\alpha} \operatorname{Hom}\left(\tilde{\mathcal{F}}, \tilde{I}_{\alpha}\right)^{*} \otimes$ $\tilde{I}_{\alpha}$ is compatible with Hodge structures, and the right hand side obviously lies in $\tilde{\mathcal{O}}_{\tilde{P}_{e}}$, so we are done.

Step 4. As follows from Step 3, the $\tilde{I}_{\alpha}(n)$ are injective objects of $\tilde{\mathcal{O}}_{\tilde{P}_{e}}$. Since $\operatorname{Irr} \tilde{\mathcal{O}}_{\tilde{P}_{e}}=\left\{\tilde{\mathcal{L}}_{\alpha}(n)\right\}$ by 4.5.3 (2), our theorem follows from 4.3.2 (2). (Strictly speaking, we need to apply it to $\mathcal{C}=\mathcal{O}^{\text {opp }}$, since in $4.3 .2(2)$ we spoke about projective objects, and here we deal with injective ones.)

4.6. Monodromy actions. We still have to prove Lemma 4.5.5. We need some general facts about monodromic sheaves. Let $H$ be our torus, $\pi: X \rightarrow Y$ an $H$-principal bundle, $\mathcal{F} \in P(X)$ a monodromic mixed Hodge module.

Proposition 4.6.1. The logarithm of the unipotent part of monodromy $\mathfrak{h}(1) \rightarrow$ End $\mathcal{F}$ is compatible with Hodge structures.

Proof. Recall that a perverse sheaf $\mathcal{F} \in \mathcal{P}(X)$ is called monodromic if $h^{*} \mathcal{F}$ is isomorphic to $\mathcal{F}$ for all $h \in H$. Consider the projection and the multiplication $p, m: H \times X \rightarrow X$ and the other projection $q: H \times X \rightarrow H$. Consider

$$
A=A_{\mathcal{F}}=H^{-n} q_{*} \mathcal{R} \operatorname{Hom}\left(p^{*} \mathcal{F}, m^{!} \mathcal{F}\right)[-n] .
$$

If $\mathcal{F}$ is monodromic, this is a local system on $H$. The fibre $A_{1}$ of this local system at one is canonically $A_{1}=\operatorname{End} \mathcal{F}$. Put $\Gamma=X_{*}(H) \subset \mathfrak{h}$. We regard this as an integral Hodge structure, and consider also its Tate twist $\Gamma(1)=2 \pi i \Gamma \subset \mathfrak{h}(1)$. Then $\Gamma(1)=\pi_{1}(H, 1)$ acts on $A_{1}=\operatorname{End} \mathcal{F}$ by monodromy, and the action of $\gamma \in \Gamma(1)$ is in fact left multiplication with some $M(\gamma) \in \operatorname{Aut} \mathcal{F}$. Now let $N(\gamma) \in \operatorname{End} \mathcal{F}$ be the logarithm of the unipotent part of $M(\gamma)$. Then $N: \Gamma(1) \rightarrow \operatorname{End} \mathcal{F}$ extends to a homomorphism of Lie algebras $N_{\mathcal{F}}: \mathfrak{h}(1) \rightarrow \operatorname{End} \mathcal{F}$, and we claim that when $\mathcal{F}$ has a mixed structure this is compatible with the mixed structures. 
But it is known that if $A$ is a smooth mixed Hodge sheaf on a torus, then the logarithm of the monodromy $N_{A}: \mathfrak{h}(1) \rightarrow$ End $A_{1}$ is compatible with Hodge structures. And when $A=A_{\mathcal{F}}\left(\right.$ so that $\left.A_{1}=\operatorname{End} \mathcal{F}\right)$ this factors as $\mathfrak{h}(1) \rightarrow \operatorname{End} \mathcal{F} \rightarrow$ $\operatorname{End}(\operatorname{End} \mathcal{F})$, where the first map is $N_{\mathcal{F}}$ and the second is left multiplication.

Proof of Lemma 4.5.5. Let $U \subset B$ be the unipotent radical. Then we may identify

$$
\begin{gathered}
U \text {-equivariant perverse sheaves on } G / B \\
\| \\
(U \times B) \text {-equivariant perverse sheaves on } G \\
\| \\
B \text {-equivariant perverse sheaves on } U / G \text {. }
\end{gathered}
$$

Certainly the latter are $H$-monodromic. Thus monodromy gives a homomorphism $\mathcal{S}(\mathfrak{h}(1)) \rightarrow$ End $\mathcal{F}$ for all $\mathcal{F} \in \mathcal{O}$, and for $\mathcal{F} \in \tilde{\mathcal{O}}$ this morphism is even compatible with Hodge structures. Now using [BB86] one may see that under localization $\mathcal{O}^{\mathfrak{b}} \cong$ $\mathcal{P}_{B}(G / B)$ the monodromy action of $\mathcal{S}(\mathfrak{h}(1))$ on the right corresponds to the action of the center of $U$ on the left, via the Harish-Chandra homomorphism. In particular for $\tilde{P}_{e} \in \tilde{\mathcal{O}}$ a lift of the antidominant projective, by translating [Soe90, Bea83] into geometry we get a surjection $\mathcal{S}(\mathfrak{h}(1)) \rightarrow \operatorname{End} \tilde{P}_{e}$. Thus indeed End $\tilde{P}_{e}$ is a split Tate structure.

\section{REFERENCES}

[ABV92] Jeffrey Adams, Dan Barbasch, and David A. Vogan, Jr, The Langlands classification and irreducible characters for real reductive groups, Progress in Mathematics, vol. 104, Birkhäuser, 1992. MR 93j:22001

[Bac82] Jörgen Backelin, A distributiveness property of augmented algebras and some related homological results, Ph. D. Thesis, Stockholm, 1982.

[Bar83] Dan Barbasch, Filtrations on Verma modules, Ann. Scient. Éc. Norm. Sup. 16 (1983), 489-494. MR 85j:17013

[Bas68] Hyman Bass, Algebraic K-theory, Benjamin, 1968. MR 40:2736

[BB81] Alexander A. Beilinson and Joseph N. Bernstein, Localisation de g-modules, C. R. Acad. Sci. Paris, Sér. 1292 (1981), 15-18. MR 82k:14015

[BB86] Alexander A. Beilinson and Joseph N. Bernstein, A proof of Jantzen conjectures, Preprint, 1986; finally published in I. M. Gelfand Seminar, Advances in Soviet Math., vol. 16, part 1, Amer. Math. Soc., Providence, RI, 1993, pp. 1-50. MR 95a:22022

[BBD82] Alexander A. Beilinson, Joseph N. Bernstein, and Pierre Deligne, Faisceaux pervers, Astérisque 100 (1982). MR 86g:32015

[Bea83] Arnault Beauville, Variétés kähleriennes dont la première classe de Chern est nulle, J. Diff. Geometry 18 (1983), 755-782. MR 86c:32030

[Ber90] Joseph N. Bernstein, Trace in categories, Operator Algebras, Unitary Representations, Enveloping Algebras and Invariant Theory (Alain Connes, Michel Duflo, Anthony Joseph, and Rudolph Rentschler, eds.), Birkhäuser, 1990, pp. 417-423. MR 92d:17010

[BF85] Jörgen Backelin and Ralf Fröberg, Koszul algebras, Veronese subrings and rings with linear resolutions, Rev. Roumaine Math. Pures Appl. 30 (1985), 85-97. MR 87c:16002

[BG80] Joseph N. Bernstein and Sergei I. Gelfand, Tensor products of finite and infinite representations of semisimple Lie algebras, Compositio Math. 41 (1980), 245-285. MR 82c: 17003

[BG86] Alexander A. Beilinson and Victor Ginsburg, Mixed categories, Ext-duality and representations (results and conjectures), Preprint, 1986.

[BGG73] Joseph N. Bernstein, Israel M. Gelfand, and Sergei I. Gelfand, Schubert cells and cohomology of the spaces $G / P$, Russian Math. Surveys 28 (1973), no. 3, 1-26. MR 55:2941

[BGG78] Joseph N. Bernstein, Israel M. Gelfand, and Sergei I. Gelfand, Algebraic bundles over $\mathbb{P}^{n}$ and problems of linear algebra, Functional Analysis and its App. 12 (1978), no. 3, 214-216. MR 80c:14010a 
[BGS88] Alexander A. Beilinson, Victor Ginsburg, and Vadim V. Schechtman, Koszul duality, Journal of Geometry and Physics 5 (1988), 317-350. MR 91c:18011

[BMS87] Alexander Beilinson, Robert MacPherson, and Vadik Schechtmann, Notes on motivic cohomology, Duke Math. Journal 54 (1987), 679-710. MR 88f:14021

[Bor84] Armand Borel (ed.), Intersection cohomology, Progress in Mathematics, vol. 50, Birkhäuser, 1984. MR 88d:32024

[CC87] Luis G. Casian and David H. Collingwood, The Kazhdan-Lusztig conjecture for generalized Verma modules, Math. Z. 195 (1987), 581-600. MR 88i:17008

[CPS91] Edward Cline, Brian Parshall, and Leonard Scott, Abstract Kazhdan-Lusztig theories, Tôhoku Math. J. (2) 45 (1993), 511-534. MR 94k:20079

[Del80] Pierre Deligne, La conjecture de Weil II, IHES Publ. Math. 52 (1980), 137-252. MR 83c: 14017

[ES87] Tom J. Enright and Brad Shelton, Categories of highest weight modules: Applications to classical hermitian symmetric pairs, Memoirs of the AMS 367 (1987). MR 88f:22052

[Har66] Robin Hartshorne, Residues and duality, Lecture Notes in Mathematics, vol. 20, Springer, 1966. MR 36:5145

[Irv88] Ron S. Irving, The socle filtration of a Verma module, Ann. Scient. Éc. Norm. Sup. 21 (1988), 47-65. MR 89h:17015

[Irv90] Ron S. Irving, A filtered category $\mathcal{O}_{s}$ and applications, Memoirs of the AMS 419 (1990). MR 90f: 17011

[Jan87] Jens C. Jantzen, Representations of algebraic groups, Pure and Applied Mathematics, vol. 131, Academic Press, 1987. MR 89c:20001

[Kel92] Bernhard Keller, Deriving DG-categories, Ann. Sci. École Norm. Sup. (4) 27 (1994), 63-102. MR 95e: 18010

[KL80a] David Kazhdan and George Lusztig, Representations of Coxeter groups and Hecke algebras, Inventiones Math. 53 (1980), 191-213. MR 81j:20066

[KL80b] David Kazhdan and George Lusztig, Schubert varieties and Poincaré duality, Proc. Symp. Pure Math. 36, AMS, 1980, pp. 185-203. MR 84g:14054

[KS90] Masaki Kashiwara and Pierre Schapira, Sheaves on manifolds, Grundlehren, vol. 292, Springer, 1990. MR 92a:58132

[Löf86] Clas Löfwall, On the subalgebra generated by the one-dimensional elements in the Yoneda ext-algebra, Lecture Notes in Mathematics, vol. 1183, Springer, 1986, pp. 291338. MR 88f: 16030

[MV87] R. Mirollo and Kari Vilonen, Bernstein-Gelfand-Gelfand reciprocity on perverse sheaves, Ann. Scient. Ec. Norm. Sup. $4^{e}$ série 20 (1987), 311-324. MR 89e:32017

[Pri70] Stewart B. Priddy, Koszul resolutions, Transactions AMS 152 (1970), 39-60. MR 42:346

[Sai90] Morihiko Saito, Mixed Hodge modules, Publications RIMS, Kyoto University 26 (1990), 221-333. MR 91m:14014

[Soe86] Wolfgang Soergel, Équivalences de certaines catégories de g-modules, C. R. Acad. Sci. Paris, Sér. 1303 (1986), no. 15, 725-728. MR 88c:17011

[Soe89a] Wolfgang Soergel, $\mathfrak{n}$-cohomology of simple highest weight modules on walls and purity, Inventiones Math. 98 (1989), 565-580. MR 90m:22037

[Soe89b] Wolfgang Soergel, Parabolisch-singuläre Dualität für Kategorie $\mathcal{O}$, Preprint MPI/89-68, 1989.

[Soe90] Wolfgang Soergel, Kategorie $\mathcal{O}$, perverse Garben und Moduln über den Koinvarianten zur Weylgruppe, Journal of the AMS 3 (1990), 421-445. MR 91e:17007

[Soe92] Wolfgang Soergel, Langlands' philosophy and Koszul duality, Preprint, 1992.

Abstract. The aim of this paper is to work out a concrete example as well as to provide the general pattern of applications of Koszul duality to representation theory. The paper consists of three parts relatively independent of each other.

The first part gives a reasonably selfcontained introduction to Koszul rings and Koszul duality. Koszul rings are certain $\mathbb{Z}$-graded rings with particularly nice homological properties which involve a kind of duality. Thus, to a Koszul ring one associates naturally the dual Koszul ring. The second part is devoted to an application to representation theory of semisimple Lie algebras. We show 
that the block of the Bernstein-Gelfand-Gelfand category $\mathcal{O}$ that corresponds to any fixed central character is governed by the Koszul ring. Moreover, the dual of that ring governs a certain subcategory of the category $\mathcal{O}$ again. This generalizes the selfduality theorem conjectured by Beilinson and Ginsburg in 1986 and proved by Soergel in 1990. In the third part we study certain categories of mixed perverse sheaves on a variety stratified by affine linear spaces. We provide a general criterion for such a category to be governed by a Koszul ring. In the flag variety case this reduces to the setup of part two. In the more general case of affine flag manifolds and affine Grassmannians the criterion should yield interesting results about representations of quantum groups and affine Lie algebras.

Department of Mathematics, Massachusetts Institute of Technology, Cambridge, MASSACHusETTS 02139

E-mail address: sasha@math.mit.edu

Department of Mathematics, The University of Chicago, Chicago, Illinois 60637 E-mail address: ginzburg@math.uchicago.edu

Max-Planck-Institut für Mathematik, Gottfried-Claren-Strasse 26, D-53 Bonn 3 , Germany

Current address: Mathematisches Institut, Universität Freiburg, Albertstraße 23b, D-79104 Freiburg, Germany

E-mail address: soergel@sun1.mathematik.uni-freiburg.de 